University of Tennessee Health Science Center

UTHSC Digital Commons

\title{
Discovery of Dihydroartemisinin and Dasatinib Drug Combination to Cure Pooroutcome BCR-ABL+ Acute Lymphoblastic Leukemia
}

Harpreet Singh

University of Tennessee Health Science Center

Follow this and additional works at: https://dc.uthsc.edu/dissertations

Part of the Medical Sciences Commons, Neoplasms Commons, Pharmaceutical Preparations Commons, and the Pharmaceutics and Drug Design Commons

\section{Recommended Citation}

Singh, Harpreet , "Discovery of Dihydroartemisinin and Dasatinib Drug Combination to Cure Pooroutcome BCR-ABL+ Acute Lymphoblastic Leukemia" (2012). Theses and Dissertations (ETD). Paper 249. http://dx.doi.org/10.21007/etd.cghs.2012.0292. 


\title{
Discovery of Dihydroartemisinin and Dasatinib Drug Combination to Cure Pooroutcome BCR-ABL+ Acute Lymphoblastic Leukemia
}

\begin{abstract}
Oncogenic signaling by the Philadelphia chromosome-encoded BCR-ABL fusion kinase initiates and drives both Philadelphia chromosome-positive acute lymphoblastic leukemia (Ph+ ALL) and chronic myelogenous leukemia (CML). Food and Drug Administration (FDA)- approved BCR-ABL-specific kinase inhibitors (BCR-ABL-KIs) imatinib, dasatinib and nilotinib induce prolonged remissions in CML but poor leukemia-reduction and relapse-control in $\mathrm{Ph}+\mathrm{ALL}$. The relative primary $\mathrm{BCR}-\mathrm{ABL}-\mathrm{KI}$-resistance in $\mathrm{Ph}+$ ALL patients carrying predominantly BCR-ABLWT disease cannot be attributed to drug-resistant BCR-ABL mutations (BCR-ABLMUTANTS), and remains poorly understood.

We established a cell-based platform to evaluate the modulation of anti-Ph+ ALL activity of drugs by both tumor-extrinsic cytokines normally present in the leukemia microenvironment and tumor-intrinsic vectormediated alteration of candidate genes. We identified that BCR-ABLWT-driven Ph+ ALL cells are rendered significantly resistant against all FDAapproved BCR-ABL-KIs by 1) Several host-cytokines, but most dominantly by Interleukin7 (IL7), and 2) Cell-intrinsic functional-loss of IKAROS. Utilizing IL7-deficient recipient mice, we demonstrated that physiological levels of IL7 significantly attenuate the survival benefit derived from dasatinib monotherapy against Ph+ ALL. Follow-up mechanistic studies using cell signaling and gene expression comparisons indicated that IL7 imparts chemo-refractoriness by initiating IL7-pSTAT5-c-MYC signaling. Interestingly, IKAROS haploinsufficiency, which has been previously associated with poor clinical prognosis in patients with $\mathrm{Ph}+\mathrm{ALL}$, was recently demonstrated to directly de-repress C-MYC expression. This suggested that both cell-extrinsic IL7 and cell-intrinsic IKAROS loss converge on c-MYC, which may act as node of leukemia BCR-ABL-KI-refractoriness. We confirmed that vector-mediated modifications imitating IL7 induction of STAT5 activity, functional-loss of IKAROS, and cMYC over-expression, all selectively enrich $\mathrm{Ph}+\mathrm{ALL}$ cells during prolonged exposures to imatinib and dasatinib, thus revealing new chemo-refractory phenotypes.

Contemporary medicine advocates co-treatment with agents of complementary mechanism of drugaction to tackle drug resistance. To identify combination agents that could overcome the dasatinibresistant phenotypes of Ph+ ALL, we screened a library of 3200 agents including known anti-infective and chemotherapy drugs. We discovered that a well known antimalarial drug dihydroartemisinin (DHA) killed host-IL7-protected BCR-ABLWT, c-MYC-overexpressing BCR-ABLWT and BCR-ABLMUTANT Ph+ ALL cells in vitro. In vivo, DHA displayed weak activity as a single agent but its addition synergistically augmented the leukemia reduction by dasatinib, relative to either of the two drugs alone. Remarkably, DHA and dasatinib combination regimen eliminated host-protected dasatinib-refractory persistent leukemia and improved long-term survival from 0 to $>90 \%$ in a murine model that faithfully captures the BCR-ABL-KI drug-refractoriness of human $\mathrm{Ph}+\mathrm{ALL}$.
\end{abstract}

This study: 1) Uncovers novel mechanisms of clinical drug-resistance against BCR-ABL-KIs, 2) Identifies increased levels of IL7, pSTAT5 and c-MYC protein, and IKAROS haploinsufficiency as potential biomarkers of BCR-ABL-targeted drug-resistance, 3) Strongly supports clinical exploration of the BCR$\mathrm{ABL}-\mathrm{KI}$ and $\mathrm{DHA}$ combinations for treating patients with $\mathrm{Ph}+\mathrm{ALL}$, and 4) Establishes a paradigm for investigating frequently overlooked host-tumor-drug interactions.

\section{Document Type}

Dissertation

Degree Name

Doctor of Philosophy (PhD) 


\section{Program}

Biomedical Sciences

Research Advisor

Gerard P. Zambetti, Ph.D. (for Richard T. Williams, M.D., Ph.D.)

\section{Keywords}

High Throughput Drug Screening, Tumor Microenvironment, Ph+ Acute Lymphoblastic Leukemia, BCR-

ABL Kinase Inhibitors, Dasatinib, Dihydroartemisinin

\section{Subject Categories}

Chemicals and Drugs | Diseases | Medical Sciences | Medicine and Health Sciences | Neoplasms |

Pharmaceutical Preparations | Pharmaceutics and Drug Design | Pharmacy and Pharmaceutical Sciences 


\title{
Discovery of Dihydroartemisinin and Dasatinib Drug Combination to Cure Poor-outcome BCR-ABL+ Acute Lymphoblastic Leukemia
}

\author{
A Dissertation \\ Presented for \\ The Graduate Studies Council \\ The University of Tennessee \\ Health Science Center
}

In Partial Fulfillment

Of the Requirements for the Degree

Doctor of Philosophy

From The University of Tennessee

By

Harpreet Singh

August 2012 
Copyright (C) 2012 by Harpreet Singh. All rights reserved. 


\section{DEDICATION}

I dedicate this dissertation to my parents, family members and close friends for their endless love, support and guidance. 


\section{ACKNOWLEDGEMENTS}

I am indebted to my mentors - Dr. Richard T. Williams and Dr. Gerard P. Zambetti, and my graduate committee members Dr.R. Kiplin Guy, Dr. Sima Jeha, Dr. Suzanne J. Baker and Dr. Rajendra S. Raghow for their guidance, constructive criticism and meaningful suggestions throughout my project for this dissertation. I would also like to express my gratitude for Dr. Asha Pillai, Dr. Pelle Holmfeldt and my brother Dr. Amandeep Singh for their remarkable scientific discussions and willing professional support, which provided me a continuous source of inspiration and motivation throughout my doctoral training.

I also thank Dr.Richard Kriwacki, Dr. Luigi Iconaru and Dr. Joseph Opfermann for the ongoing scientific collaborations. I am also thankful to Chandra Savage, Branden Williams and Dr. Nidal Boulos for assistance with mouse therapeutic studies, Dr. Christopher R. Calabrese and Monique Payton for assistance with animal imaging, Alexander J. Kovalic for assistance with in vitro growth factor screening experiments, Dr. Narendra P. Singh for suggestions on drug-preparation for animal use, Dr. Armand Guiguemde and Jimmy Cui at the High Throughput Screening Center at St. Jude for assistance with high-throughput drug screening, Heather Mulder for performing BCRABL KD mutation analyses, Richard A. Ashmun and Jim Houston for performing flow cytometric cell-sorting and analyses, Dr. Jianmin Wang at Department of Information Sciences for statistical studies, and Dr. Charles G. Mullighan and Dr. Owen Witte (UCLA) for providing the retroviral vectors. This work was supported in part by American Association for Cancer Research (AACR) Centennial Career Development Award for Childhood Cancer Research and Gabrielle's Angel Foundation Medical Research Award for Dr. Richard T. Williams, NIH (1R21NS066460-01), NIH/NCI Comprehensive Cancer Center Core Grant (CA-21765) and by the American and Lebanese Syrian Associated Charities (ALSAC) of St. Jude Children's Research Hospital.

I also thank the administration and faculty at the University of Tennessee Health Science Center (UTHSC) and St. Jude Children's Research Hospital (SJCRH) for

providing me an opportunity to study science and a wonderful learning experience. 


\begin{abstract}
Oncogenic signaling by the Philadelphia chromosome-encoded BCR-ABL fusion kinase initiates and drives both Philadelphia chromosome-positive acute lymphoblastic leukemia (Ph+ ALL) and chronic myelogenous leukemia (CML). Food and Drug Administration (FDA)-approved BCR-ABL-specific kinase inhibitors (BCR-ABL-KIs) imatinib, dasatinib and nilotinib induce prolonged remissions in CML but poor leukemiareduction and relapse-control in $\mathrm{Ph}+\mathrm{ALL}$. The relative primary BCR-ABL-KI-resistance in $\mathrm{Ph}+\mathrm{ALL}$ patients carrying predominantly $\mathrm{BCR}-\mathrm{ABL}^{\mathrm{WT}}$ disease cannot be attributed to drug-resistant BCR-ABL mutations (BCR-ABL ${ }^{\text {MUTANTS }}$ ), and remains poorly understood.

We established a cell-based platform to evaluate the modulation of anti-Ph+ ALL activity of drugs by both tumor-extrinsic cytokines normally present in the leukemia microenvironment and tumor-intrinsic vector-mediated alteration of candidate genes. We identified that BCR-ABL ${ }^{\mathrm{WT}}$-driven $\mathrm{Ph}+\mathrm{ALL}$ cells are rendered significantly resistant against all FDA-approved BCR-ABL-KIs by 1) Several host-cytokines, but most dominantly by Interleukin7 (IL7), and 2) Cell-intrinsic functional-loss of IKAROS. Utilizing IL7-deficient recipient mice, we demonstrated that physiological levels of IL7 significantly attenuate the survival benefit derived from dasatinib monotherapy against $\mathrm{Ph}+\mathrm{ALL}$. Follow-up mechanistic studies using cell signaling and gene expression comparisons indicated that IL7 imparts chemo-refractoriness by initiating IL7-pSTAT5c-MYC signaling. Interestingly, IKAROS haploinsufficiency, which has been previously associated with poor clinical prognosis in patients with $\mathrm{Ph}+\mathrm{ALL}$, was recently demonstrated to directly de-repress c-MYC expression. This suggested that both cellextrinsic IL7 and cell-intrinsic IKAROS loss converge on c-MYC, which may act as node of leukemia BCR-ABL-KI-refractoriness. We confirmed that vector-mediated modifications imitating IL7 induction of STAT5 activity, functional-loss of IKAROS, and c-MYC over-expression, all selectively enrich $\mathrm{Ph}+\mathrm{ALL}$ cells during prolonged exposures to imatinib and dasatinib, thus revealing new chemo-refractory phenotypes.

Contemporary medicine advocates co-treatment with agents of complementary mechanism of drug-action to tackle drug resistance. To identify combination agents that could overcome the dasatinib-resistant phenotypes of Ph+ ALL, we screened a library of 3200 agents including known anti-infective and chemotherapy drugs. We discovered that a well known anti-malarial drug dihydroartemisinin (DHA) killed host-IL7-protected BCR-ABL ${ }^{\mathrm{WT}}$, c-MYC-overexpressing BCR-ABL ${ }^{\mathrm{WT}}$ and BCR-ABL ${ }^{\mathrm{MUTANT}} \mathrm{Ph}+\mathrm{ALL}$ cells in vitro. In vivo, DHA displayed weak activity as a single agent but its addition synergistically augmented the leukemia reduction by dasatinib, relative to either of the two drugs alone. Remarkably, DHA and dasatinib combination regimen eliminated host-protected dasatinib-refractory persistent leukemia and improved long-term survival from 0 to $>90 \%$ in a murine model that faithfully captures the BCR-ABL-KI drug-refractoriness of human $\mathrm{Ph}+\mathrm{ALL}$.
\end{abstract}

This study: 1) Uncovers novel mechanisms of clinical drug-resistance against BCR-ABL-KIs, 2) Identifies increased levels of IL7, pSTAT5 and c-MYC protein, and 
IKAROS haploinsufficiency as potential biomarkers of BCR-ABL-targeted drug-resistance, 3) Strongly supports clinical exploration of the BCR-ABL-KI and DHA combinations for treating patients with $\mathrm{Ph}+\mathrm{ALL}$, and 4) Establishes a paradigm for investigating frequently overlooked host-tumor-drug interactions. 


\section{TABLE OF CONTENTS}

\section{CHAPTER 1. INTRODUCTION ...................................................................................1}

Target Focused Therapies in New Era Oncology and Road to Cure ..............................

Trends of Drug Discovery Industry in Post-imatinib Era ........................................ 1

Current Clinical Status of Target Focused Drugs in Oncology ...................................1

Targeted Therapy against Philadelphia Chromosome-positive $(\mathrm{Ph}+)$ Leukemias .........2

The Philadelphia Chromosome ......................................................................

Rationale and Agents for BCR-ABL Targeted Therapy........................................

Outcomes of BCR-ABL Targeted Therapy in CML ..............................................

Outcomes of BCR-ABL Targeted Therapy in Ph+ ALL ..........................................

Deficiencies of Current BCR-ABL Inhibition Therapy .............................................4

BCR-ABL Kinase Domain Mutations Mediate BCR-ABL-KI Drug Resistance .......4

Primary Drug Resistance against BCR-ABL-KIs Is Independent of BCR-ABL

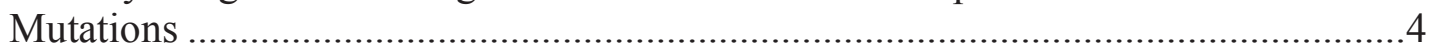

$\mathrm{Ph}+$ ALL Faithfully Captures the Deficiencies of Targeted Therapy .........................5

An Accurate Murine Model System of Human Ph+ ALL .............................................5

Etiology of Clinical BCR-ABL ${ }^{\text {MUTANT }}$-independent BCR-ABL-KI-resistance ...........6

\section{CHAPTER 2. HYPOTHESIS AND SPECIFIC AIMS ............................................8}

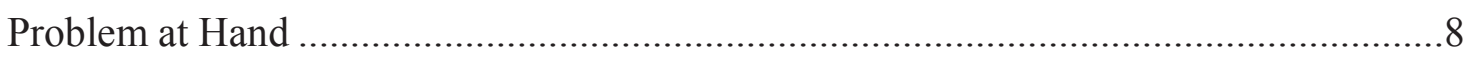

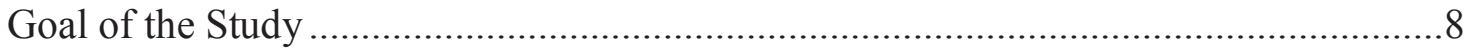

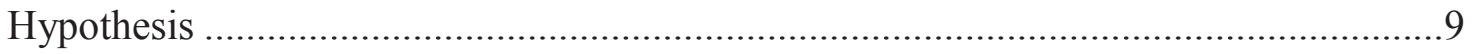

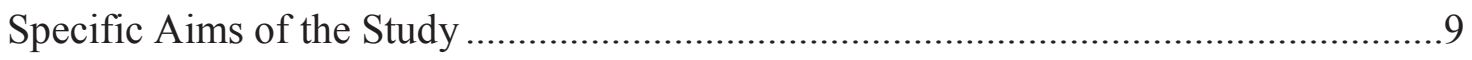

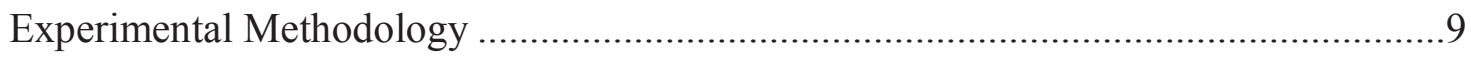

CHAPTER 3. MATERIALS AND METHODS.................................................................11

Production and Culture of Murine Leukemia-initiating Cells (LICs) ...........................11

In Vivo Adoptive Leukemia Transfer Model of Ph+ ALL ..........................................11

FACS Assessment of Viability ............................................................................ 11

FACS Assessment of Cell Cycle Percentages .......................................................... 12

Development of LIC-based Multimodality Screening Assay ....................................... 12

Forward Cytokine Phenotypic Screen and Other In Vitro Cellular Assays ...................16

Drug/Cytokine Washout and Cytokine Recovery Studies..........................................16

Dasatinib Re-challenge Studies ..................................................................... 19

High-throughput (HT) Phenotypic Drug Screening ................................................19

Description of Chemical Library Screened ...............................................................20

Data Processing, Quality Control and Hit Scoring Criteria for HT Drug Screens .......20

Cluster Analysis of Hits Using Therapeutic Classes .............................................22 
Evaluation of Hits for Tolerance in Human Patients and Selectivity for MYC Gene

Expression in Their Mechanism of Drug Action Using Public Databases ...................22

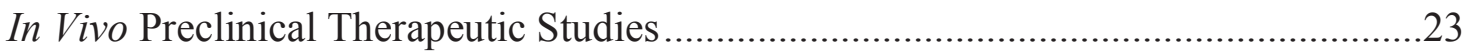

Bioluminescent Imaging to Track Therapeutic Responses In Vivo ................................23

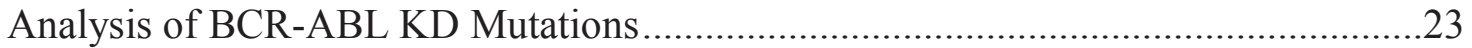

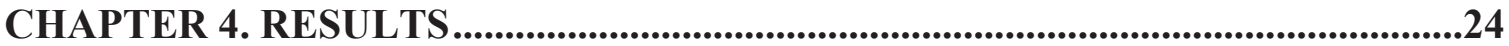

A BCR-ABL Kinase Domain Mutation Does Not Impart Any Advantage in Leukemia Progression In Vitro or In Vivo

Cytokine Screen Identifies IL7 as the Main Host Factor That Mediates Primary

Dasatinib Resistance, Independent of BCR-ABL Mutations

IL7 Mediates Primary Drug Resistance against All Approved BCR-ABL-KIs in Murine and Human Ph+ ALL Cells, Which Is Completely Reversible

Physiological Levels of Host-expressed IL7 Contributes to an Aggressive, Dasatinib-resistant Phenotype of $\mathrm{Ph}+\mathrm{ALL}$ In Vivo .....

IL7 Protects against Dasatinib by Strongly Counteracting the Decrease in pSTAT5

Protein and c-MYC Expression

High pSTAT5 and IKAROS Haploinsufficiency Are Candidate Resistance

Biomarkers with c-MYC as a Convergence Node of Resistance against BCR-ABL

Inhibitor Therapy in $\mathrm{Ph}+\mathrm{ALL}$

HT Drug Screens to Identify Agents with Activity against Ph+ ALL Cells

Protected by Extracellular IL7 and T315I Mutation.....

A Common Anti-malarial Agent DHA Overcomes Multi-factorial BCR-ABL-KI

Drug Resistance by Cell-extrinsic IL7 and Cell-intrinsic c-MYC Over-expression ....36

Dihydroartemisinin Possesses Single-agent In Vivo Activity against Ph+ ALL

Phenotypes Demonstrating Primary and Acquired Dasatinib Resistance .....

Cotreatment with Dihydroartemisinin Overcomes Host-mediated Primary Drug-

resistance against Dasatinib and Eliminates $\mathrm{Ph}+\mathrm{ALL}$ In Vivo

CHAPTER 5. DISCUSSION AND CONCLUSIONS ......................................................53

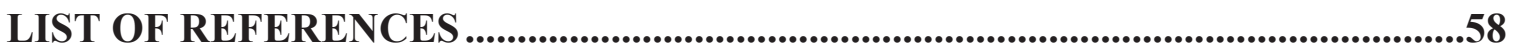

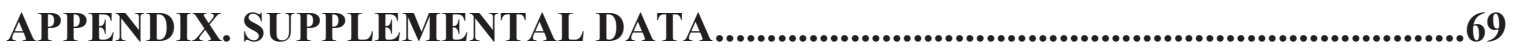

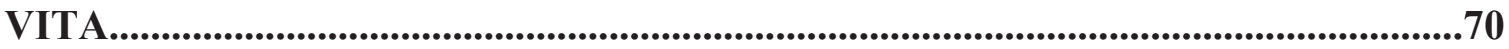




\section{LIST OF TABLES}

Table 3.1. Steps in optimized high-throughput screening assay (CTG readout)..........17

Table 4.1. Priortization of validated hits according to known human tolerability and predicted involvement of c-MYC expression in their anti-cancer drug action. 


\section{LIST OF FIGURES}

Figure 3.1. Comparison of the growth properties of $A r f^{/-} \mathrm{p} 185^{\mathrm{WT}}$ (filled symbols) and $\mathrm{Arf}^{\prime-}$ p $185^{\mathrm{T} 3151}$ pre-B (empty symbols) populations during in vitro cell culture.

Figure 3.2. Optimization of the high-throughput LIC-based phenotypic assay...............14

Figure 3.3. Optimization of HT-flow output to perform drug competition assays. ........15

Figure 4.1. Leukemia stem cell (LSC) function assay by serial dilution and implantation comparing the in vivo leukemia-initiating capacity of $\mathrm{Arf}^{-1}$ $\mathrm{p} 185^{\mathrm{WT}}$ (left) and $A r f^{\prime-}$ p $185^{\mathrm{T} 315 \mathrm{I}}$ pre-B (right) populations.........................25

Figure 4.2. IL7 confers BCR-ABL ${ }^{\mathrm{MUTANT}}$-independent primary drug-resistance against BCR-ABL-KIs in Ph+ ALL.......................................................26

Figure 4.3. Comparative evaluation of IL7-mediated protection conferred against non-BCR-ABL-specific targeting drugs (top panel) and conventional cytotoxic antileukemic clinical drugs (bottom panel)... .28

Figure 4.4. Protection of $A r f^{/-}$BCR-ABL ${ }^{\mathrm{WT}}$ LICs by IL7 during dasatinib exposures ( 0 to $72 \mathrm{~h}$ ) and in the post-exposure recovery period (72 to $144 \mathrm{~h}$, no dasatinib).

Figure 4.5. In vitro leukemia clonal outgrowth efficiency assays. 30

Figure 4.6. Mechanistic evaluation of host-IL7 imparted primary resistance against dasatinib.

Figure 4.7. Results of counter genetic screen by HT-flow confirming the role of pSTAT5 activity, IKAROS haploinsufficiency and c-MYC overexpression in primary drug resistance against BCR-ABL-inhibiting drugs.

Figure 4.8. Quality control for the primary screen of 5600 agents against $\mathrm{Arf}^{/-}$ BCR-ABL ${ }^{\mathrm{WT}}$ LICs performed in the presence of $0.85 \mathrm{ng} / \mathrm{mL}$ IL7.

Figure 4.9. Quality control for primary screen of 5600 agents against $\mathrm{Arf}^{\prime-}$ BCR$\mathrm{ABL}^{\mathrm{T} 315 \mathrm{I}} \mathrm{LICs}$ performed in the presence of $0.85 \mathrm{ng} / \mathrm{mL}$ IL 7 .

Figure 4.10. Primary high-throughput drug screening experiments captured BCR-ABL-KI drug-resistant phenotypes with high accuracy. 
Figure 4.11. Receiver Operating Characteristic (ROC) analysis demonstrates that both (a) $\mathrm{Arf}^{-1}$ BCR-ABL ${ }^{\mathrm{WT}}$ and (b) $\mathrm{Arf}^{-1}$ BCR-ABL ${ }^{\mathrm{T} 315 \mathrm{I}}$ LIC-based assays have high discriminatory power (AUC 0.83 and 0.85 , respectively).

Figure 4.12. Frequency of ATC Level 1 (top) and ATC Level 2 (bottom) compounds for the 706 compounds submitted to secondary analysis.

Figure 4.13. High-throughput drug screen to identify novel agents possessing activity against IL7-mediated early (primary) and BCR-ABL ${ }^{\text {MUTANT }}$-mediated late (secondary) BCR-ABL-KI drug-resistant phenotypes.

Figure 4.14. Dihydroartemisinin of Artemisinin family of antimalarials circumvents dasatinib resistance induced by IL7, c-MYC overexpression and T315I mutation in vitro.

Figure 4.15. Dihydroartemisinin is active as a single agent against dasatinib-resistant $\mathrm{Ph}+\mathrm{ALL}$ in $\mathrm{IL} 7^{+/+}$host mice.

Figure 4.16. Dihydroartemisinin works synergistically with dasatinib to cure $\mathrm{Ph}+$ ALL by improving primary responses to dasatinib.

Figure 4.17. Statistical analyses to predict the activity of dihydroartemisin against human $\mathrm{Ph}+\mathrm{ALL}$ 


\section{CHAPTER 1. INTRODUCTION}

\section{Target Focused Therapies in New Era Oncology and Road to Cure}

Ongoing efforts to understand critical signaling pathways that undergo deregulation during tumorigenesis identify specific oncogenes and driver mutations integral for the sustenance and proliferation of various cancer types [1-2]. The development of agents that modulate such key molecular targets should improve patient tolerance and cure rates over conventional non-specific chemotherapy by selectively killing cancer cells [1-3].

\section{Trends of Drug Discovery Industry in Post-imatinib Era}

Imatinib (Gleevec by Novartis) is the first example of a cancer-specific small molecule inhibitor that was ever approved for clinical use, in particular to treat human CML. Initial clinical success of imatinib in dramatically controlling the previously poor outcome CML marked a paradigm shift in our approach to anti-cancer therapy and jump-started drug discovery efforts against an ever-growing list of validated molecular targets in other cancers [4-8]. Since then, remarkable progress has been made in the field of target focused drug discovery [9-11] especially for agents that particularly inhibit deregulated receptor or non-receptor kinase proteins [8, 10-14], similar to imatinib, and are commonly referred to as kinase inhibitors (KIs). The fast-pace development of KIs is facilitated by the chemically druggable structure of kinase proteins, advances in computational modeling, medicinal chemistry and high throughput biophysical assays that compare binding of chemicals against normal and deregulated kinases [5, 15-20]. Current automated drug pipelines against diverse kinases and non-kinase targets generate hundreds of potential leads, providing tremendous hope to patients suffering from diverse leukemias and solid tumors worldwide [5, 9-10, 14, 21].

\section{Current Clinical Status of Target Focused Drugs in Oncology}

Rational target focused drug discovery has tremendously decreased the total time required between the initial identification of a potential agent and its actual testing in cancer patients. Within the last decade, multiple cancer-selective drugs and biological agents have been formally approved and many more are being evaluated in clinical trials, namely against $\mathrm{CML}, \mathrm{Ph}+\mathrm{ALL}$, gastro-intestinal stromal tumors, non-small cell lung carcinoma, melanoma, sarcoma, renal cancers etc. However, the majority of cancer-selective agents often induce suboptimal primary responses and offer meaningful benefit in only a sub-set of cancer patients, which frequently lasts only for a short period of time [7, 9-10, 12, 14, 22-23]. Most frustratingly, all current agents which kill the respective cancer cells with potent selective activities in vitro, invariably fail to eradicate the same cancer cells expressing intact molecular targets in human patients $[7,9-10,12$, 14, 22-24]. As a consequence, many pre-clinical agents have failed to produce 
meaningful long-term clinical benefit to the majority of patients despite expected pharmacodynamic changes in the intended molecular targets in vivo [7, 9-10, 12, 14, 2224], and as a consequence, a few agents have been completely dropped. Since negative results are often not reported, the number of such failed agents is bound to be much higher.

Paradoxical clinical (in vivo) drug-resistance observed against available cancerselective agents in general, remains poorly understood. It likely stems from our limited experience with this modern class of oncology agents and has to be addressed to improve long term cancer outcomes.

\section{Targeted Therapy against Philadelphia Chromosome-positive $(\mathrm{Ph}+)$ Leukemias}

From among the available cancer targeting drugs, the most extensive preclinical and clinical data is available for imatinib and imatinib-like drugs, primarily for their use against a set of leukemias defined by a unique cytogenetic abnormality - Philadelphia Chromosome [25-26].

\section{The Philadelphia Chromosome}

The Philadelphia chromosome is the hallmark of CML and a high-risk subset of acute lymphoblastic leukemia (Ph+ ALL). The Philadelphia chromosome arises from a balanced translocation between chromosomes 9 and 22 [25-26]. It encodes an abnormal fusion protein, BCR-ABL, which plays an essential role in initiating and driving both $\mathrm{CML}$ and $\mathrm{Ph}+\mathrm{ALL}$ [25-26]. The oncogenic potential of BCR-ABL protein arises from the constitutive activation of the tyrosine kinase domain $(\mathrm{KD})$ of $\mathrm{ABL}$ in the fused protein configuration.

In CML, mainly the $\mathrm{p} 210^{\mathrm{BCR}-\mathrm{ABL}}$ isoform is expressed in hematopoietic stem cells (HSCs) which are conceptually referred to as leukemia stem cells (LSCs) [27], and in both myeloid and lymphoid progeny. Whereas in $\mathrm{Ph}+\mathrm{ALL}$, expression of mainly $\mathrm{p} 185^{\mathrm{BCR}-\mathrm{ABL}}$ or alternative $\mathrm{p} 210^{\mathrm{BCR}-\mathrm{ABL}}$ isoform is restricted to the lymphoid B cell lineage, which display robust LSC-like function to both initiate and propagate leukemia during serial transplantation assays.

\section{Rationale and Agents for BCR-ABL Targeted Therapy}

The required role for $\mathrm{BCR}-\mathrm{ABL}$ in sustaining $\mathrm{Ph}+$ leukemias, an attribute referred to as 'oncogene addiction', rationalizes the inhibition of BCR-ABL as an attractive therapeutic strategy in patients with CML and Ph+ ALL [25-26]. 
The design of imatinib allows it to selectively bind to the KD of ABL and inhibit the constitutive tyrosine kinase activity of BCR-ABL fusion protein. Imatinib was initially approved against CML alone [28]. Currently, three BCR-ABL-specific kinase inhibitors (BCR-ABL-KIs) namely imatinib (first generation), nilotinib and dasatinib (second generation) are FDA-approved for use against both CML and Ph+ ALL. First and second generation BCR-ABL-KIs differ in their chemical structures, pharmacokinetic properties, binding sites on the ABL, potency to inhibit ABL kinase activity, spectrum of off-target inhibition of other kinases and associated adverse effects [20].

\section{Outcomes of BCR-ABL Targeted Therapy in CML}

At the time of diagnosis, CML most commonly is detected in its chronic (early) phase and less frequently in accelerated or blast (advanced) phase. In most of the CML patients suffering from chronic phase of the disease, continuous BCR-ABL-KI monotherapy (using imatinib and more recently dasatinib and nilotinib) induces dramatic and long-lasting remissions [29-30]. The majority of patients who have received long-term treatments with BCR-ABL-KIs show strong leukemia control by cytogenetic evaluation [26, 29, 31-32], and similar results are observed in animal models [33]. However, almost invariably patients test positive for mRNA transcripts of BCR-ABL (approx 97\%) [34], indicating that leukemia has not been completely eliminated [31]. The dormant leukemia cells which evade eradication by BCR-ABL-KIs in CML have been shown to express the characteristic surface markers of LSCs, and have been proposed to sustain drug resistant disease, in a fashion similar to HSCs [35-36] and independent of BCR-ABL kinase [37]. Discontinuation of BCR-ABL-KI treatment often results in clinical relapse and hematopoietic stem cell transplantation (HSCT) still remains important modality for treating those patients who have accelerated phase and blast crisis CML or those who either do not tolerate or fail/relapse during continuous BCR-ABL-KI treatments [38-41].

\section{Outcomes of BCR-ABL Targeted Therapy in Ph+ ALL}

In contrast to the robust cytogenetic and molecular responses in CML patients, BCR-ABL-KI monotherapy evokes much weaker and short lasting responses in patients with $\mathrm{Ph}+\mathrm{ALL}$ [42]. These differences are also appreciated in animal leukemia models $[33,43]$. Despite receiving continuous BCR-ABL-KI therapy, human patients with $\mathrm{Ph}+\mathrm{ALL}$ typically carry significantly higher levels of persistent leukemia and inevitably relapse within a few months [42]. The relative primary drug-resistance in human $\mathrm{Ph}+\mathrm{ALL}$ necessitates the addition of several chemotherapeutic agents and HSCT in combination with BCR-ABL-KIs [44]. Multi-modality BCR-ABL-KI-combined regimens, the current standard of clinical care in $\mathrm{Ph}+\mathrm{ALL}$, improve the remission rates but relapses remain common [44-47] with an 18- to 24-month survival rate of $64 \%$ and an even lower relapse-free survival rate [44]. 
The leukemia cells in $\mathrm{Ph}+\mathrm{ALL}$ which display poor initial response and evade eradication by BCR-ABL-KIs [45-47] express cell surface markers of lymphoid B cell lineage [48]. No distinct population of LSCs has been characterized to drive drug-resistant populations, unlike CML [35-36, 48].

\section{Deficiencies of Current BCR-ABL Inhibition Therapy}

\section{BCR-ABL Kinase Domain Mutations Mediate BCR-ABL-KI Drug Resistance}

The most frequently studied mechanism of drug-resistance against BCR-ABL-KIs is point mutations that occur within the KD of $\mathrm{ABL}\left(\mathrm{BCR}-\mathrm{ABL}{ }^{\text {MUTANTS }}\right.$ ), which is common to both CML and Ph+ ALL [49-52]. Mechanistically, the mutations in $\mathrm{BCR}-\mathrm{ABL}$, especially in the $\mathrm{KD}$, deteriorate the drug-binding and rescue the oncogenic kinase from drug-induced inhibition [49-51]. The total number of the distinct ABL KD mutations that have been characterized from refractory $\mathrm{Ph}+$ leukemia samples now approaches 80 [53]. The spectrum of BCR-ABL mutations that each of the BCR-ABL-KIs select in $\mathrm{Ph}+$ leukemias is distinct, but overlapping, with the notable exception of the T315I (Threonine to Isoleucine) mutation which confers profound cell-intrinsic resistance to all three approved BCR-ABL-KIs.

Rigorous research efforts are being made to design the next generation agents which can mitigate the effect of BCR-ABL mutations on drug-binding and thus achieve sustained BCR-ABL inhibition in vivo, as a critical step towards improvement in the clinical efficacy of BCR-ABL targeted therapy [17-18].

\section{Primary Drug Resistance against BCR-ABL-KIs Is Independent of BCR-ABL Mutations}

According to the long-term and metadata studies, drug-resistant BCR-ABL mutations are predominantly absent in: i) $\mathrm{Ph}+\mathrm{ALL}$ at the time of diagnosis which responds poorly to BCR-ABL-KIs [50-51,54], and ii) persistent $\mathrm{Ph}+$ leukemias (both $\mathrm{CML}$ and $\mathrm{Ph}+\mathrm{ALL}$ ) which evade eradication during the first few months of targeted therapy [49-52]. Periodic testing reveals that as high as $50 \%$ of the $\mathrm{Ph}+$ leukemia patients never test positive for drug resistant BCR-ABL mutations. Mutations in the ABL KD when detected for the first time are quantitatively present as a minority leukemia sub-population. Frequency and population size of mutations increases after prolonged period of treatment with BCR-ABL-KIs $[50-52,54]$. On the other hand, ABL KD mutations can spontaneously regress in patients when the BCR-ABL-KI is changed, implicating BCR-ABL inhibition in the selective evolution of drug-resistant mutations.

Thus, primary clinical drug resistance against BCR-ABL-KIs occurs in the absence of well characterized ABL KD mutations, and likely plays an instrumental role in overall therapeutic failures [49-52]. 


\section{Ph+ ALL Faithfully Captures the Deficiencies of Targeted Therapy}

Approved BCR-ABL-KIs potently inhibit the KD of ABL protein in biochemical assays and in vivo [55] and commit $\mathrm{Ph}+$ leukemia cells to death in vitro [56]. In stark contrast, due to unknown reasons, BCR-ABL-KIs fail to completely eliminate leukemia in patients with Ph+ ALL $[42,44]$ and CML [31]. While the drug refractoriness mediated by $\mathrm{ABL} \mathrm{KD}$ mutations is common to both $\mathrm{CML}$ and $\mathrm{Ph}+\mathrm{ALL}$, poor responses to BCR-ABL-KIs during early therapy, when patients carry predominantly non-mutated BCR-ABL (BCR-ABL ${ }^{\mathrm{WT}}$ ) leukemia, are clearly more evident in patients with $\mathrm{Ph}+\mathrm{ALL}$ compared to CML.

Thus, Ph+ ALL faithfully captures both the primary and target-acquired (ABL KD mutations) mechanisms of therapeutic failures against BCR-ABL-KIs. These features qualify $\mathrm{Ph}+\mathrm{ALL}$ as an ideal disease system to study and delineate the deficiencies of imatinib, nilotinib and dasatinib - the drugs which have played an instrumental role in qualifying the use of target focused cancer therapy in clinical settings.

Elaboration of the deficiencies of BCR-ABL-KIs may hold general lessons for further optimization and development of alternative targeted therapies in other cancers.

\section{An Accurate Murine Model System of Human Ph+ ALL}

In generating and validating an accurate model of human $\mathrm{Ph}+\mathrm{ALL}$ that recapitulates key features of the human disease, we previously demonstrated that p $185^{\mathrm{BCR}-\mathrm{ABL}}$ expression potently synergized with inactivation of the Arf tumor suppressor gene $\left(\mathrm{Arf}^{--}\right)$; these two engineered genetic events are sufficient to fully transform murine pre-B cells into leukemia-initiating cells (LICs) [43]. The BCR-ABL oncogenic kinase potently 'drives' the proliferation of primary $\mathrm{Arf}^{\prime-} \mathrm{p} 185+$ pre-B cells in a growth factor independent manner, while the loss of Arf function dramatically attenuates oncogeneinduced, p53-dependent apoptosis, confers cellular immortalization and facilitates exponential, high-viability growth. In this leukemia model system, the Arf-loss dependent phenotype closely matches that associated with engineered loss of the p53 tumor suppressor, thus Arf and p53 do essentially function in a 'linear' pathway [57-58]. The process of BCR-ABL mediated transformation of pre-B cells is dependent upon cooperation of transcription factors - signal transducer and activator of transcription (STAT5) [59] and c-MYC [60-62], a known oncogene. BCR-ABL fusion protein phosphorylates the STAT5 protein (pSTAT5) in the cytoplasm which results in its dimerization and transport to the nucleus where it binds to specific STAT5 response elements in the promoter region of target genes, including c-MYC.

More recently, this murine $\mathrm{Ph}+\mathrm{ALL}$ model was adapted for in vivo luminescent imaging to quantitatively monitor leukemic cell dissemination and therapeutic responses, using Arf $^{/-}$p185 ${ }^{\text {BCR-ABL }}$ luciferase-expressing (Luc+) pre-B or LICs [57, 63]. As described in human $\mathrm{Ph}+\mathrm{ALL}$ clinical trials, single-agent dasatinib therapy in this murine 
model induces transient remissions in recipient mice harboring very high leukemic burdens, only to be followed by clinical relapse a few weeks later (i.e. acquired dasatinib resistance) which is frequently associated with the emergence of BCR-ABL kinase domain mutations in cells recovered from leukemic-infiltrated tissues [57, 63]. However, dasatinib-refractory leukemic cells recovered from leukemic recipients at the end of 'induction' (i.e. at the nadir of leukemic burden, after maximal drug response had been achieved) infrequently harbored BCR-ABL kinase domain mutations [63], strongly implicating that BCR-ABL ${ }^{\text {MUTANT }}$-independent mechanisms initially attenuate dasatinib's ability to fully eradicate this minimal residual disease (MRD).

\section{Etiology of Clinical BCR-ABL ${ }^{\text {MUTANT }}$-independent BCR-ABL-KI-resistance}

While rigorous studies has been overtaken to study BCR-ABL mutation mediated drug resistance [51, 53, 64-65], sparse efforts have been made to address and overcome the primary BCR-ABL-KI-resistance which is predominantly BCR-ABL ${ }^{\text {MUTANT }}$ independent. Primary BCR-ABL-KI-resistance can originate from factors that can broadly be classified as either leukemia-extrinsic or leukemia-intrinsic.

Among leukemia-extrinsic influences, tumor microenvironment [36, 66-67] and poor availability of drug in the target tissues can play a role. Though the tumor microenvironment can profoundly alter the drug-action against the tumor in vivo, its influence is conventionally not addressed during in vitro drug-evaluations [66]. Cell-extrinsic soluble factors such as GM-CSF and GM-CSF plus G-CSF in human CML, IL3 in myeloid and lymphoid systems, common gamma chain cytokine signaling and its family member IL7 in lymphoid pre-B cells, significantly attenuate BCR-ABL-KI induced leukemia cell killing [48, 62, 66, 68-69]. This suggests that signaling pathways activated by host growth factors can play a role in drug resistance in BCR-ABL driven leukemias in vivo. On the other hand, primary chemo-refractoriness may also arise from poor drug-availability in specific target tissues that harbor leukemic cells [70-71]. Both $\mathrm{CML}$ and $\mathrm{Ph}+\mathrm{ALL}$ are primarily disorders of hematopoietic tissues and share a majority of the sites of spread, namely, bone marrow, lymph nodes, spleen, liver and thymus [26]. Unfavorable pharmacokinetic properties, which are inherent to the chemical nature of drug, are thus less likely to play an important role in the varied responses to BCR-ABL-KI agents in Ph+ ALL and CML.

Cell-intrinsic drug resistance mechanisms that function independent of BCR-ABL mutations include genetic factors, epigenetic changes or altered dynamics of drug import and drug efflux that modulate intracellular inhibitor concentrations. Studies by others have established that genetic deletions involving the tumor suppressor $C D K N 2 A / B$ [7274], lymphoid transcriptional regulator IKAROS (also called IKZF1) [75-76], PAX5 [73], EBF1 [73], cooperate with BCR-ABL in leukemogenesis. These genetic events, which are involved in the pathological development of disease, are present at the time of diagnosis in a majority of the patients with Ph+ ALL [74-76] but not CML patients. They 
may also play a role in poor primary responses to BCR-ABL-KIs in $\mathrm{Ph}+\mathrm{ALL}$, but this hypothesis remains to be ascertained. 


\section{CHAPTER 2. HYPOTHESIS AND SPECIFIC AIMS}

\section{Problem at Hand}

Elucidation of the mechanisms of resistance against clinically used drugs, especially the new era target-selective agents, is integral to improve their efficacy and achieve long-term survival in cancer patients. Significantly, primary drug resistance against BCR-ABL-KIs in human Ph+ ALL very well represents the clinical dilemma shared by a majority of current drugs that selectively target and kill cancer cells in vitro but not in vivo. This disparity strongly implicates the modulation of therapeutic response of tumor cells within the host microenvironment.

In contrast to the remarkable susceptibility of BCR-ABL ${ }^{\mathrm{WT}}$ cells to $\mathrm{BCR}-\mathrm{ABL}-\mathrm{KIs}$ in vitro, patients suffering from $\mathrm{Ph}+\mathrm{ALL}$ receive poor and transient benefit from BCR-ABL-KI therapy despite the predominant absence of ABL-KD mutations at diagnosis and during initial few months of therapy. The poor primary responses evoked by BCR-ABL-KI monotherapy necessitates clinical management of $\mathrm{Ph}+\mathrm{ALL}$ patients with a multi-modality approach, which is often complicated by the frequent unavailability of HSCT donors and the toxicity/intolerability of conventional chemotherapy and HSCT, especially in the elderly who form majority of the Ph+ ALL population. The performance of BCR-ABL-KIs deteriorates even further later during the therapy when ABL KD mutations appear in leukemia lymphoblasts, imparting molecular drug resistance.

While wide scientific attention is paid to improve the drug design for tackling molecular drug resistance, it appears clinically more relevant to mechanistically understand why BCR-ABL ${ }^{\mathrm{WT}} \mathrm{Ph}+\mathrm{ALL}$ responds poorly and evades eradication by BCR-ABL-KIs prior to the development of molecular resistance mediated by KD mutations. The unforeseen primary (BCR-ABL mutation-independent) resistance is most likely imparted by the tumor microenvironment (leukemia-extrinsic) and/or alternatively by changes within the tumor itself (leukemia-intrinsic). We previously reported a role for host cytokines of the common gamma chain receptor family in mediating primary imatinib resistance [48], but many alternative host-derived growth factors have similarly been proposed in BCR-ABL-KI drug resistance [62, 66-69]. Currently, no consensus exists for the relative importance of cytokines that control the cellular responses to BCR-ABL targeted agents.

\section{Goal of the Study}

The central goal of this scientific work was to advance the performance of BCR-ABL targeted therapy in $\mathrm{Ph}+\mathrm{ALL}-$ a human leukemia that currently has poor long-term survival outcomes. 


\section{Hypothesis}

Defining and countering the factors that confer the poor response to BCR-ABL-KIs in patients with non-mutated BCR-ABL (BCR-ABL ${ }^{\mathrm{WT}}$ ) $\mathrm{Ph}+\mathrm{ALL}$ is critical to harness the full therapeutic potential of targeting BCR-ABL for improving the long-term survival. We hypothesized that - 'Combination therapy with drugs that are active against the $\mathrm{Ph}+\mathrm{ALL}$ phenotypes displaying mechanisms of primary drug resistance can eradicate leukemias that are refractory to BCR-ABL-KIs in vivo.'

\section{Specific Aims of the Study}

To maximize the likelihood of discovering combination agents that may overcome multi-faceted BCR-ABL-KI drug resistance in $\mathrm{Ph}+\mathrm{ALL}$ in vivo, we aimed to:

i. Create a rank-order of leukemia microenvironment-based host-cytokine/s for imparting primary drug resistance (cell-extrinsic) against current FDA-approved $\mathrm{BCR}-\mathrm{ABL}-\mathrm{KI}$ agents in vitro.

ii. Test the most relevant host-cytokine/s from (i) for conferring BCR-ABL-KIrefractory phenotype in vivo and characterize its associated cell-intrinsic changes.

iii. Screen drug-rich chemical libraries to identify agents that possess in vitro activity against $\mathrm{Ph}+\mathrm{ALL}$ cells protected by cell-extrinsic host-cytokine(s) (from i and ii) and previously known molecular mechanisms (T315I) of BCR-ABL-KI drug-resistance.

iv. Test agents (from iii) alone and in combination with dasatinib in our murine model of $\mathrm{Ph}+\mathrm{ALL}$. Assess agent's ability to improve the primary responses to dasatinib and eradicate dasatinib refractory persistent disease, as a critical step towards their evaluation in patients with $\mathrm{Ph}+\mathrm{ALL}$ clinics.

\section{Experimental Methodology}

Here, we report efforts to establish a high throughput (HT) cell-based platform that captures the dominant cell - extrinsic (host environment imparted) and intrinsic (BCR-ABL mutation-dependent) mechanisms of BCR-ABL-KI-refractoriness in $\mathrm{Ph}+$ $\mathrm{ALL}$, in order to discover therapeutic agents capable of improving in vivo BCR-ABL-KI performance.

Utilizing Arf-null ( $\left.\operatorname{Arf}^{\prime-}\right)$ BCR-ABL transformed LICs, which model key biological and genetic features of human $\mathrm{Ph}+\mathrm{ALL}$, we developed an assay that evaluates

drug performance against $\mathrm{BCR}-\mathrm{ABL}^{\mathrm{WT}}$ LICs co-cultured with candidate cell-extrinsic cytokines and growth factors. 
Following an unbiased screening approach, we identified IL7 as a specific hostcytokine that conferred primary chemo-refractoriness against all FDA-approved BCRABL-KIs in vitro, and subsequently confirmed these clinically-relevant results in vivo. Next, we assayed this drug-resistant phenotype against a library of FDA-approved drugs, natural products, and chemical tool compounds. In addition to the identification of many clinical anti-leukemic and other chemotherapeutic agents, several novel agents overcame the BCR-ABL-KI-resistant phenotype. Testing of combinations of dasatinib with one of our most compelling screening hits, the FDA-approved antimalarial drug dihydroartemisinin (DHA), eradicated the residual pool of dasatinib-refractory leukemia in vivo. This treatment protocol durably cured $90 \%$ of treated animals. This opens up a promising therapeutic avenue for treating human $\mathrm{Ph}+\mathrm{ALL}$ patients by adding DHA to current dasatinib regimens, at the time of diagnosis. 


\section{CHAPTER 3. MATERIALS AND METHODS}

\section{Production and Culture of Murine Leukemia-initiating Cells (LICs)}

Replication-defective mouse stem cell virus (MSCV) retroviral vectors coexpressing either wild-type (WT) allele of human $\mathrm{p} 185^{\mathrm{BCR}-\mathrm{ABL}}$ or mutant BCR-ABL alleles, namely $\mathrm{p} 185^{\mathrm{T} 315 \mathrm{I}}$ or $\mathrm{p} 185^{\mathrm{F} 317 \mathrm{~L}}$, and either green fluorescent protein $(\mathrm{GFP})$ or luciferase, were packaged into replication-incompetent ecotropic virions [43, 48, 63]. Whole bone marrow cell suspensions from $\mathrm{Arf}^{\prime-}$ young adult mice were transduced to produce $\mathrm{Arf}^{\prime-}$ p185+ pre-B cells $[43,63]$. After initial establishment on autologous stromal cell layers over 7 days, transformed pre-B cells were briefly expanded in the absence of a stromal layer for 2 days in liquid culture in BCM10 media (RPMI1640 supplemented with 10\% Hyclone fetal calf serum, $4 \mathrm{mM}$ glutamine, 100 Units $/ \mathrm{mL}$ penicillin, $100 \mu \mathrm{g} / \mathrm{mL}$ streptomycin, and $55 \mu \mathrm{M}$ beta-mercaptoethanol) [48], and cryopreserved as 40 identical vials per cell line, generating a master cell bank. Arf $^{-1-}$ p185 ${ }^{\mathrm{WT}}$ and $A r f^{\prime-}$ p $185^{\mathrm{T} 315 \mathrm{I}}$ pre-B cells (BCR-ABL ${ }^{\mathrm{WT}}$ LICs and BCR-ABL ${ }^{\mathrm{T} 15 \mathrm{I}}$ LICs, respectively) from the cell bank were thawed and allowed to recover in BCM10 for 3 days, prior to their use in any in vitro or in vivo assays.

MSCV-IRES-GFP retroviral vectors expressing human c-Myc or empty 'control', and MSCV-IRES-GFP or MSCV-IRES-RFP retroviral vectors expressing previouslyvalidated mutants of IKAROS (either IK6 or IK1) [75] or constitutively active (CA)STAT5 mutants [77], were utilized to transduce BCR-ABL-expressing LICs, and cultured for 48 in BCM10 media before use in drug competition assays.

\section{In Vivo Adoptive Leukemia Transfer Model of Ph+ ALL}

Mice were housed in an American Association of Laboratory Animal Care (AALAC)-accredited facility and treated on Institutional Animal Care and Use Committee (IACUC)-approved protocols in accordance with NIH guidelines. LICs were injected into tail veins of healthy, non-conditioned, immune-competent 10- to 12-weekold IL $7^{+/+}$or IL $7^{-/-} \mathrm{C} 57 \mathrm{Bl} / 6 \mathrm{~J}$ mice (Jackson Labs, Bar Harbor, ME). Animals were observed daily and were sacrificed when moribund (dehydration, ruffled fur, poor mobility, respiratory distress). Survival curves were generated using GraphPad Prism Version 5.0 (La Jolla, CA). Mantel-Cox test was applied to pair-wise comparisons of survival data.

\section{FACS Assessment of Viability}

Viability of LICs was estimated after counterstaining approximately $5 \times 10^{5}$ LICs (in $0.5 \mathrm{~mL}$ volume) with $35 \mu \mathrm{L}$ of a propidium iodide (PI) solution $(0.25 \mathrm{mg} / \mathrm{mL}$ in PBS). The percentage of viable cells was calculated by electronic gating on PI-negative cells on PI versus forward scatter dot plots, with forward scatter signals consistent with LICs, and 
comparison of this number with total cell number. Data were collected and analyzed on a BD Biosciences LSR II flow cytometer (San Jose, CA), using BD Biosciences FACS DiVa software.

\section{FACS Assessment of Cell Cycle Percentages}

For determination of percentages in cell cycle phases, $5 \times 10^{5}$ LICs were centrifuged, washed once with PBS, recentrifuged, and cell pellets were resuspended in $0.5 \mathrm{~mL}$ PI staining solution $(0.05 \mathrm{mg} / \mathrm{mL}$ PI, $0.1 \%$ sodium citrate, $0.1 \%$ Triton X100), which lysed the cells and stained nuclear DNA with PI. Samples were then treated with DNAse-free RNAse $(0.2 \mathrm{mg} / \mathrm{mL}$ in $10 \mathrm{M}$ Tris- $\mathrm{HCl} / 15 \mathrm{mM} \mathrm{NaCl}, \mathrm{pH} 7.5)$ for $30 \mathrm{~min}$ at room temperature, filtered, and analyzed for red fluorescence from a PI-labeled DNA on BD Biosciences FACS Calibur flow cytometer (San Jose, CA) by using BD Biosciences CellQuest Pro software. The percentages of cells within each cell cycle phase were computed using the computer program ModFit (Verity Software House, Topsham, ME).

\section{Development of LIC-based Multimodality Screening Assay}

Before use in any assay, LICs from the master cell bank had to pass the following quality control parameters: test thawing (benchmark: $95 \%$ viability at $24 \mathrm{~h}$ ); PCR genotyping to confirm $\mathrm{Arf}^{\prime-}$ status [78]; mycoplasma testing (negative by Takara Bio Mycoplasma PCR assay, Clontech, Madison WI); sequence verification of BCR-ABL allele [79]; and in vitro dasatinib sensitivity.

Maximum DMSO concentration tolerated by LICs in culture was determined to be $0.2 \%$ (by volume) and was always maintained $\leq 0.1 \%$ (by volume). LICs plated in 384-well plates at a cellular density of $5 \times 10^{4}$ per $\mathrm{mL}$ in BCM10 were confirmed to give exponential and reproducible growth during the first $72 \mathrm{~h}$ (Figure 3.1). To select assay reagents, 3 high throughput screening (HTS) relevant cellular assays were evaluated according to manufacturer's instructions (Figure 3.2), and flow cytometry analysis was performed in parallel (as a reference control) using a high-throughput sampling device (Hypercyte by Intellicyte Corporation, Albuquerque, NM) and BD Biosciences LSR II flow cytometer (San Jose, CA). Relative to accuracy of high throughput flow cytometry (HT-flow) to detect low number of viable LICs, especially at high drug concentrations, the luminescent CellTiter-Glo (CTG)-assay by Promega Madison, WI, outperformed a common spectrophotometry-based methane-thiosulfonate-based (MTS) reagent (CellTiter $96^{\circledR}$ AQueous One Solution Cell Proliferation assay; Promega Madison, WI), and alamar blue (not shown). Since CTG-assay monitors LIC proliferation by using total ATP content as a surrogate marker, it inherently cannot distinguish between genetically distinct sub-populations within the same well. To adapt the evaluation of cell-intrinsic genetic factors into our drug screening platform, cell-mixtures of $\mathrm{Arf}^{\prime-} \mathrm{BCR}-\mathrm{ABL}^{\mathrm{WT}}$-RFP LICs and $\mathrm{Arf}^{\prime-}$ BCR-ABL ${ }^{\mathrm{T} 15 \mathrm{I}}$-GFP LICs were treated with dasatinib (Figure 3.3) and HT-flow assay performed after $72 \mathrm{hr}$ drug exposures. HT-flow (drug-challenge competition assay) correctly detected the expected dasatinib-induced selective 


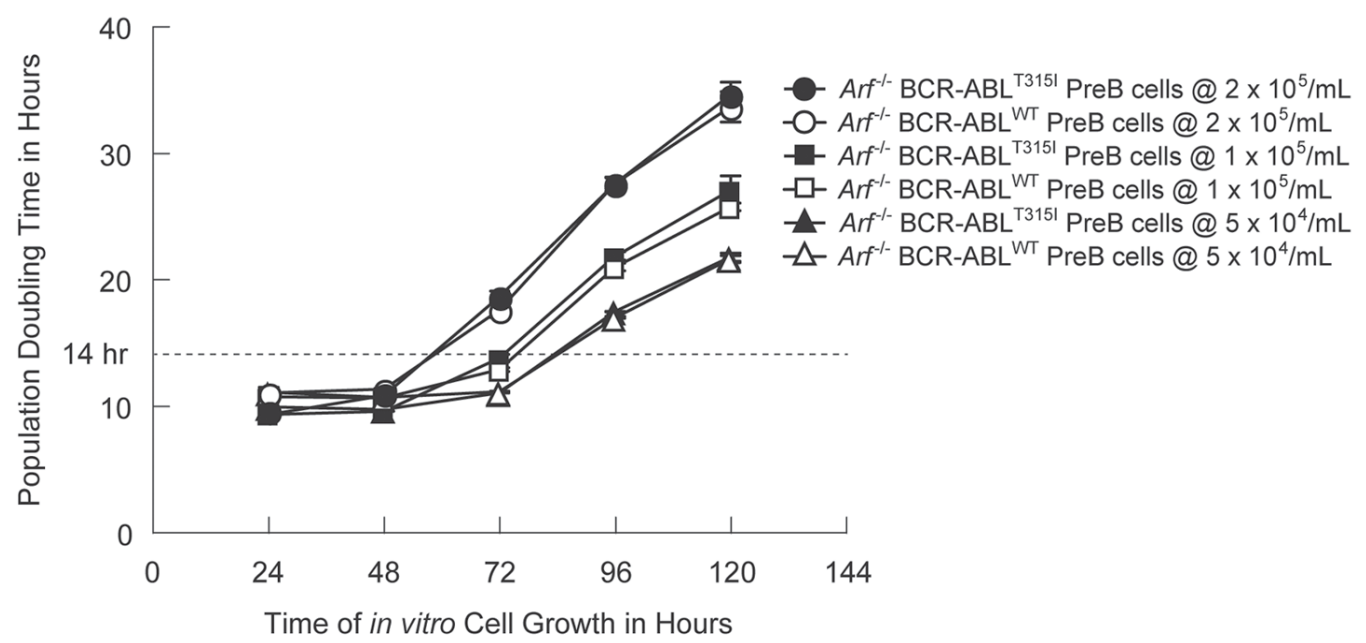

Figure 3.1. Comparison of the growth properties of $\mathrm{Arf}^{-/} \mathrm{p} 185^{\mathrm{WT}}$ (filled symbols) and $\mathrm{Arf}^{--}$p185 $^{\mathrm{T} 315 \mathrm{I}}$ pre-B (empty symbols) populations during in vitro cell culture.

Both cell types were plated at multiple (eight) cell dilutions ( $\mathrm{n}=3$ for each condition, time $=0 \mathrm{~h}$ ) in 6-well plates and serially followed daily for 5 days to evaluate absolute viable cell count, total viability, doubling times, and total population doublings, using the Beckman Coulter cell counter (trypan blue staining) and fluorescence-activated cell sorting (FACS) analysis in parallel. Both cell types had identical growth properties. Only the comparison for population doubling time in hours $(+/-\mathrm{sd})$ for 3 relevant plating densities is presented. FACS cell-cycle analysis confirmed that a population doubling time less than $14 \mathrm{~h}$ was sufficient to maintain cells in exponential growth $(>50 \%$ cells in S-phase). 

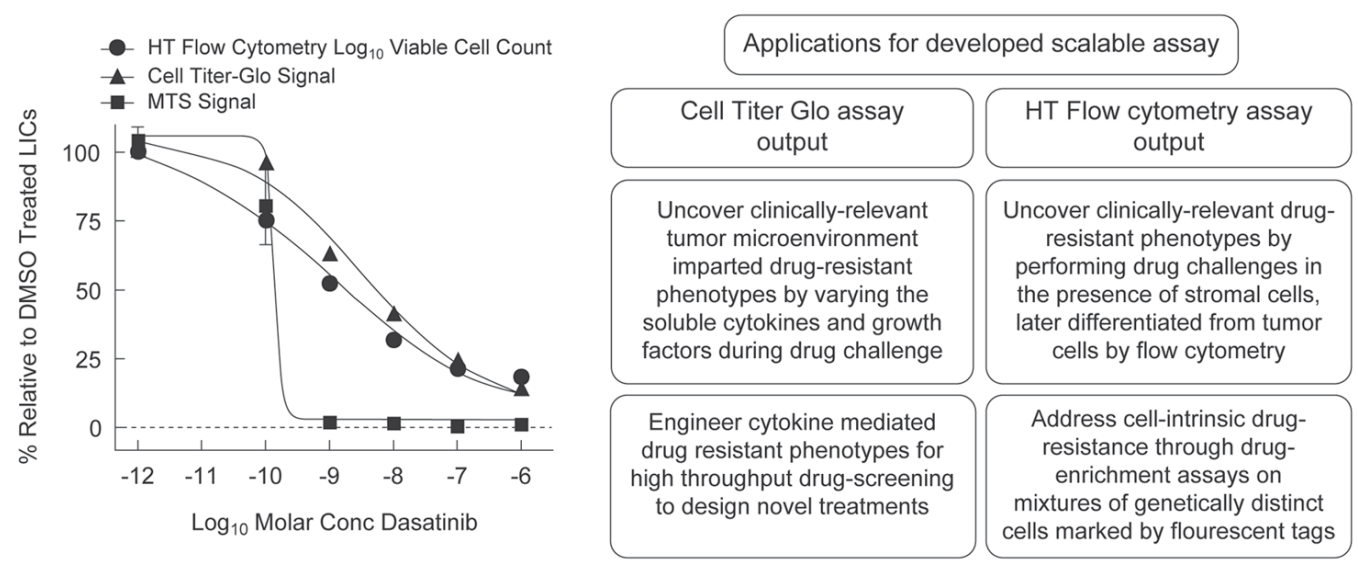

Figure 3.2. Optimization of the high-throughput LIC-based phenotypic assay.

$\mathrm{Arf}^{\prime-}$ BCR-ABL ${ }^{\mathrm{WT}}$ LICs plated at $5 \times 10^{4}$ per $\mathrm{mL}$ were challenged with 6 log-fold concentration range of dasatinib or DMSO in 384-well microplates. After a $72 \mathrm{~h}$ drug challenge, LIC growth was measured by 3 cellular readouts: CellTiter-Glo (CTG)-assay (ATP content), MTS assay (viability), and flow cytometry (total viability and viable cell counts by DAPI exclusion) in high-throughput (HT-flow) mode. Measured assay readouts at various drug concentrations ( $\mathrm{x}$-axis) were normalized to DMSO-treated LICs and graphed $(+/-$ sd) on y-axis. Total viable LIC counts were determined by FACS, reflecting dasatinib-induced phenotypic changes across a 6 log-fold drug-concentration, were closely paralleled by the CTG cellular assay output but not by the commonly used MTS assay. 
a

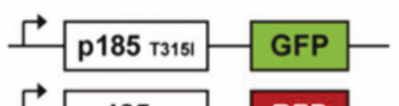

p185 wT

RFP

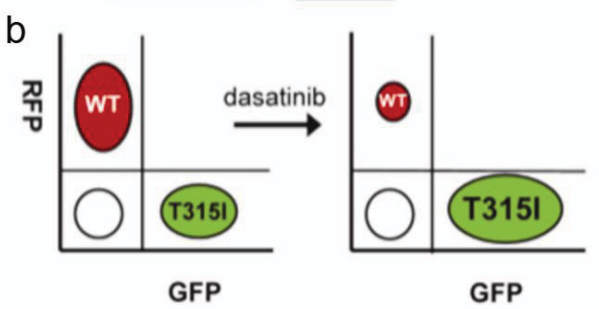

C

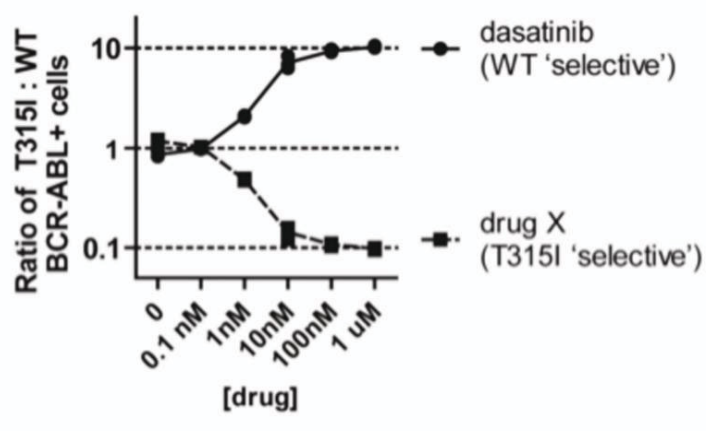

Figure 3.3. Optimization of HT-flow output to perform drug competition assays.

(a) $\mathrm{Arf}^{/-}$BCR-ABL ${ }^{\mathrm{WT}}$ LICs and $\mathrm{Arf}^{/-}$BCR-ABL ${ }^{\mathrm{T} 315 \mathrm{I}}$ LICs were mixed in 1:1 ratio at time $\mathrm{t}=0 \mathrm{hrs}$ and treated with dasatinib under optimized assay conditions. Schematic of BCR-ABL alleles in LICs is presented. (b) Schematic of set-up of flow cytometry analysis at $\mathrm{t}=72 \mathrm{hrs}$ (c) Change in ratio of BCR-ABL ${ }^{\mathrm{WT}}$ LICs to BCR-ABL ${ }^{\mathrm{T} 315 \mathrm{I}}$ LICs in response to increasing drug concentration at $\mathrm{t}=72 \mathrm{hrs}$. Real experimental data are plotted for dasatinib $(n=3)$ and a hypothetical drug $X$ that is T315I selective is depicted for comparison. 
enrichment of $\mathrm{Arf}^{--}$BCR-ABL ${ }^{\mathrm{T} 315 \mathrm{I}}$ LICs within the same well. Thus, HT-flow assay complements the CTG-assay readout by quantifying the impact of 'engineered' cell-intrinsic change towards drug refractoriness within the same well. Of the two, CTG-assay is easy to use in HTS process (add and read one-step assay with no prolonged incubation step required between reagent addition and assay readout), has 10000 fold dynamic range, measures $\mathrm{IC}_{50}$ shifts of $\leq 2$ fold for dasatinib, displays minimal drift or edge effects and robust inter-plate and inter-day reproducibility.

\section{Forward Cytokine Phenotypic Screen and Other In Vitro Cellular Assays}

Lyophilized murine and human recombinant cytokines (R\&D Systems, Minneapolis, MN) were diluted with $1 \mathrm{mg} / \mathrm{mL}$ BSA in sterile PBS to a final concentration $20 \mu \mathrm{g} / \mathrm{mL}$ and stored at $4^{\circ} \mathrm{C}$. Anti-leukemia drugs, BCR-ABL-KIs and non-BCR-ABL-specific compounds (LC labs, MA and Sigma, MO), were solubilized in DMSO to obtain $10 \mathrm{mM}$ stocks concentrations. Subsequent serial titrations were prepared using DMSO in $0.5 \mathrm{~mL}$ vials or 384 -well drug master plates, and stored at $-20^{\circ} \mathrm{C}$.

To study the impact of murine cytokines on dasatinib action, LIC stocks containing different concentrations of candidate cytokines were prepared in multichannel sterile reservoirs and plated into 384-well plates manually using handheld Finnpipette ${ }^{\circledR}$ Novus (Thermo Scientific, Waltham, MA) electronic 16 channel pipette. Drug delivery to cell suspensions with a V \& P Scientific (San Diego, CA) pin tool and all subsequent steps of the CTG-assay were performed under optimized automated conditions, as described in Table 3.1. In a forward candidate approach, evaluation of IL7's ability to attenuate drug-induced cell killing and growth inhibition was later extended to all BCR-ABL-KIs and non-BCR-ABL specific drugs using the CTG-assay in semi-automated mode (non-automated cell-plating as described above).

Human SUP-B15 Ph+ ALL cells (DSMZ cell culture collection, Germany) in McCoys media containing 20\% fetal calf serum and penicillin/streptomycin (Invitrogen, Carlsbad, CA), were plated at 12,500 cells in $25 \mu \mathrm{L}$ per well in black clear-bottom 384-well microplates. Drugs were transferred by pin-transfer. After $72 \mathrm{~h}$ incubation at $37^{\circ} \mathrm{C}, 20 \%$ (by vol) MTS assay reagent was added. Microplates were incubated for 75 min at $37^{\circ} \mathrm{C}$, and read colorimetrically at $490 \mathrm{~nm}$.

\section{Drug/Cytokine Washout and Cytokine Recovery Studies}

For washout studies, BCR-ABL ${ }^{\mathrm{WT}}$ LICs were diluted to a density of $1 \times 10^{5}$ $\mathrm{LICs} / \mathrm{mL}$ in BCM10 containing no or $5 \mathrm{mg} / \mathrm{mL}$ IL7, and treatment with either dasatinib or $0.1 \%$ DMSO by volume (non-drug treated controls) was initiated at time $0 \mathrm{~h}$ in triplicate $100 \mathrm{~mm}$ petri dishes. Daily monitoring for expected drug-induced changes was performed by naked-eye microscopy from 0 to $72 \mathrm{~h}$. At the $72 \mathrm{~h}$ time point, after harvesting under identical conditions, total LIC density and viability percentage for all samples were determined using Beckman Coulter Vi-cell (trypan blue) in triplicates. 


\section{Table 3.1. Steps in optimized high-throughput screening assay (CTG readout).}

\begin{tabular}{|c|c|c|c|}
\hline Step & Parameter & Value & Description \\
\hline 1 & Plate cells & $25 \mu \mathrm{L}$ & $\begin{array}{l}\text { Plate LICs } 5 \times 10^{4} \text { per } \mathrm{mL}(25 \mu \mathrm{L} / \text { well) in BCM10 }+0.85 \mathrm{ng} / \mathrm{mL} \text { IL7 into } 384 \text {-well } \\
\text { opaque bottom white microplates using Wellmate (Matrix) }\end{array}$ \\
\hline \multirow[t]{6}{*}{2} & \multirow{3}{*}{$\begin{array}{l}\text { Primary drug } \\
\text { screening controls } \\
\text { in columns 1-2 and } \\
13-14\end{array}$} & \multirow[t]{3}{*}{$25 \mathrm{~nL}$} & $\begin{array}{l}\text { 1A-1I: Nine dasatinib concentrations }(100 \mathrm{nM}, 30 \mathrm{nM}, 10 \mathrm{nM}, 3 \mathrm{nM}, 1 \mathrm{nM}, 0.3 \mathrm{nM} \text {, } \\
0.1 \mathrm{nM}, 0.03 \mathrm{nM} \text { and } 0.01 \mathrm{nM})- \text { control for IL7- and BCR-ABL mutation-imparted } \\
\text { resistance against BCR-ABL-KIs }\end{array}$ \\
\hline & & & $\begin{array}{l}\text { 2A-2I: Nine staurosporine concentrations }(300 \mathrm{nM}, 100 \mathrm{nM}, 60 \mathrm{nM}, 30 \mathrm{nM}, 10 \mathrm{nM}, 6 \\
\mathrm{nM}, 3 \mathrm{nM}, 1 \mathrm{nM}, 0.6 \mathrm{nM}) \text { - non-BCR-ABL-specific reference control in the presence } \\
\text { of IL7 }\end{array}$ \\
\hline & & & $\begin{array}{l}\text { 13A-13P, 1J, 2J: DMSO 0.1\% (Max Signal OR Negative Control); 14A-14P: } \\
\text { staurosporine at } 300 \mathrm{nM} \text { (Min Signal OR Positive Control); 1K-1P, 2K-2P: } 40 \mu \mathrm{M} \\
\text { dasatinib }\end{array}$ \\
\hline & \multirow{3}{*}{$\begin{array}{l}\text { Secondary drug } \\
\text { screening controls } \\
\text { in columns } 21-24\end{array}$} & \multirow[t]{3}{*}{$25 \mathrm{~nL}$} & $\begin{array}{l}\text { 21A-21P: DMSO 0.1\% (Max Signal); 22A-22P, 23K-24P, 24K-24P: staurosporine at } \\
300 \mathrm{nM} \text { (Min Signal) }\end{array}$ \\
\hline & & & $\begin{array}{l}\text { 23A-23J: Staurosporine }-10 \text { log-fold dilutions }(3 \mu \mathrm{M} \text { to } 0.3 \mathrm{pM})-\text { non-BCR-ABL- } \\
\text { specific reference control in the presence of IL7 }\end{array}$ \\
\hline & & & $\begin{array}{l}\text { 24A-24J: Dasatinib - } 10 \text { log-fold dilutions }(1 \mu \mathrm{M} \text { to } 0.1 \mathrm{pM})-\text { control for IL } 7 \text { - and } \\
\text { BCR-ABL mutation-imparted BCR-ABL-KI drug-resistance }\end{array}$ \\
\hline
\end{tabular}




\section{Table 3.1. Continued.}

\begin{tabular}{|c|c|c|c|}
\hline Step & Parameter & Value & Description \\
\hline \multirow[t]{3}{*}{3} & \multirow{3}{*}{$\begin{array}{l}\text { Add test } \\
\text { compounds in } \\
\text { columns } 3-12 \text { and } \\
15-24 \text { in primary; } \\
\text { columns } 1-20 \text { in } \\
\text { secondary screen }\end{array}$} & \multirow[t]{3}{*}{$25 \mathrm{~nL}$} & Primary screening: Single final drug concentration of $10 \mu \mathrm{M}$ \\
\hline & & & $\begin{array}{l}\text { Secondary screening: Triplicate ten half-log serial dilutions }(5 \mu \mathrm{M} \text { to } 2 \mathrm{nM} \text { final } \\
\text { concentration) of primary screening hits to determine their half-maximal inhibitory } \\
\text { concentrations }\left(\mathrm{IC}_{50}\right) \text { against LICs }\end{array}$ \\
\hline & & & $\begin{array}{l}384 \text {-well master drug-stock plates prepared at } 1000 \mathrm{X} \text { in DMSO. Drug delivered to } \\
\text { assay plates by pin transfer (using V\&P Scientific pin tool, } 10 \mathrm{H} \text { pins), giving a final } \\
\text { drug concentration of } 1 \times \text { and } 0.1 \% \text { DMSO in all control and test wells of assay plates }\end{array}$ \\
\hline 4 & Incubation 1 & $72 \mathrm{~h}$ & Liconics incubator, $8 \% \mathrm{CO}_{2}, 37^{\circ} \mathrm{C}$ (standard LIC culture conditions) \\
\hline 5 & Incubation 2 & $20 \mathrm{~min}$ & Room temp, low light (instructions as per Promega) \\
\hline 6 & Add reagent & $25 \mu \mathrm{L}$ & CellTiter Glo (Promega) pre-equilibrated to RT before use \\
\hline 7 & Incubation 3 & $25 \mathrm{~min}$ & Room temp, low light (per Promega) \\
\hline 8 & Assay readout & $\begin{array}{l}\text { ATP } \\
\text { levels }\end{array}$ & $\begin{array}{l}\text { Envision, Luminescent mode - ATP levels as a measure of LIC number and viable } \\
\text { growth in } 72 \mathrm{~h} \text { - Signal measured in RLUs }\end{array}$ \\
\hline 9 & Data processing & $\begin{array}{l}\text { Pipeline } \\
\text { Pilot }\end{array}$ & $\begin{array}{l}\% \text { inhibition by test compound }=100 *\left(\log _{10} \text { Test Signal mean }-\log _{10} \text { Min Signal }\right. \\
\text { mean }) /\left(\log _{10} \text { Min Signal mean }-\log _{10} \text { Max Signal mean }\right) \\
\text { Z-prime }\left(Z^{\prime}\right)=1-\left[\left(3^{*} \text { stdev of } \log _{10} \text { Max Signal }+3^{*} \text { stdev of } \log _{10} \text { Min }\right.\right. \\
\left.\text { Signal }) /\left(\log _{10} \text { Max Signal mean }-\log _{10} \text { Min Signal mean }\right)\right] Z^{\prime} \text { is a dimensionless } \\
\text { metric to assess assay quality }- \text { for a good assay } Z^{\prime} \geq 0.5 \text {, and for a perfect assay } Z^{\prime}=1\end{array}$ \\
\hline
\end{tabular}


Each sample was spun down in 50 mL BD Falcon ${ }^{\text {TM }}$ tubes; the supernatant was discarded and LIC pellet resuspended in freshly prepared BCM10 (not containing any cytokine or drug). This washing step was repeated 4 times to ensure complete removal of the drug and/or cytokine. After the last wash, each pellet was resuspended to $1 \times 10^{6}$ total LICs per $\mathrm{mL}$ master stocks. Aliquots from master stocks were taken for (i) flow assessment of viability and cell cycle percentages (as described above); (ii) manual CTG-assay using 25 $\mu \mathrm{L}$ sample per well in 384-well microtiter plates (in triplicates), as an independent determination for LIC growth. For assessment of extracellular cytokine on recovery of LICs that were previously challenged with dasatinib (0 to $72 \mathrm{~h}$ ), washed master stocks were diluted to $5 \times 10^{5}$ total LICs per $\mathrm{mL}$ in BCM10 with or without $5 \mathrm{ng} / \mathrm{mL}$ IL7, plated in 6 well plates $\left(10 \mathrm{~cm}^{2}\right.$ approx.), and incubated at $37^{\circ} \mathrm{C}$ from 72 to $144 \mathrm{~h}$ for recovery, during which time daily naked-eye microscopic examinations were performed. At $144 \mathrm{~h}$, for each sample total LIC density and viability percentage assessment by Beckman, viability and cell cycle percentages by flow assessment and independent CTG evaluations were performed as described for the $72 \mathrm{~h}$ time point. Trends in cell were also measured by CTG readout and used to independently corroborate the findings from trypan blue (Beckman) and DAPI staining (FACS). Total viable LICs in a sample were calculated as a product of absolute LIC count determined by Beckman and absolute sample viability determined by FACS analysis.

\section{Dasatinib Re-challenge Studies}

Further studies were performed to evaluate the possibility of a genetically drug-resistant subpopulation that could undergo selection with dasatinib/IL7 co-treatment during 0-72 $\mathrm{h}$ of dasatinib/cytokine washout studies above. To this aim, BCR-ABL ${ }^{\mathrm{WT}}$ LICs were treated with $100 \mathrm{nM}$ dasatinib or $0.1 \%$ DMSO in the absence or presence of 5 $\mathrm{ng} / \mathrm{mL}$ IL7 in $100 \mathrm{~mm}$ dishes from 0-72 $\mathrm{h}$, as described above; in addition, a dasatinib challenge (first-time challenge) across 6 log-fold concentration in the presence or absence of $5 \mathrm{ng} / \mathrm{mL}$ IL7 was also performed using CTG-assay during this time period (0-72 $\mathrm{h})$. At $72 \mathrm{~h}$, dasatinib/IL7 co-treated LICs from $100 \mathrm{~mm}$ dishes were washed (as described above) and immediately challenged (second challenge or re-challenge) with six log-fold concentration range of dasatinib in 384-well microtiter plates either in the presence or absence of $5 \mathrm{ng} / \mathrm{mL}$ IL7 from 72 to $144 \mathrm{~h}$. At 144 h, CTG-assay was performed.

\section{High-throughput (HT) Phenotypic Drug Screening}

Minimal concentration of IL7 that conferred near-maximal drug resistance against dasatinib, nilotinib and imatinib in BCR-ABL ${ }^{\mathrm{WT}}$ LICs was first determined by performing cytokine titration assays $(0.85 \mathrm{ng} / \mathrm{mL}$ IL 7$)$ under semi-automated mode and later confirmed to reproduce results under fully-automated HT screening conditions (Table 3.1). Primary single-point $(10 \mu \mathrm{M})$ HT evaluation of 5600 compounds ( 3200 unique) and subsequent secondary drug screens across 10 two-fold drug dilutions were performed. In addition to BCR-ABL ${ }^{\mathrm{WT}}$ and $\mathrm{BCR}-\mathrm{ABL}^{\mathrm{T} 315 \mathrm{I}} \mathrm{LICs}$, secondary drug-screens were also performed against $\mathrm{BCR}-\mathrm{ABL}^{\mathrm{F} 317 \mathrm{~L}} \mathrm{LICs}$, another clinically-known dasatinib- 
resistant $\mathrm{ABL} \mathrm{KD}$ mutation which gave identical results to that for $\mathrm{BCR}-\mathrm{ABL}^{\mathrm{T} 315 \mathrm{I}}$ (not shown). To track the quality of screening experiments, multiple control assay plates were set-up during all screening runs to allow for inter-plate and inter-day comparisons at 0 , 24, 48 and $72 \mathrm{hrs}$.

\section{Description of Chemical Library Screened}

The screening library consisted of 5600 (approximately 3200 unique) approved drugs and chemicals with known biological activity (bioactives). The library was assembled from 3 commercial suppliers: Microsource, Prestwick, and Sigma. The Microsource compounds included the following: (a) the Spectrum collection, which contains 2000 biologically active and structurally diverse compounds, including known drugs, experimental bioactives, and pure natural products [80-81]; (b) the US Drug Collection, which contains 1040 drugs that have reached clinical trials in the US and have been assigned USAN or US Pharmacopeia status; and (c) the Killer collection, which contains a reference set of 160 synthetic and natural toxic substances (http://www.msdiscovery.com/index.html). The Prestwick compounds include 1120 small molecules selected for high chemical and pharmacologic diversity. Ninety percent of the collection is composed of known marketed drugs, and the remainder includes bioactive alkaloids or related substances. Human bioavailability and human toxicity data are available for most compounds (http://www.prestwickchemical.fr/index.php?pa=26). The Sigma LOPAC1280 (Library of Pharmacologically Active Compounds) collection reflects the most commonly screened targets in the drug discovery community, including marketed drugs, failed development candidates, and "gold standards" that have well-characterized activities (http://www.sigmaaldrich.com/chemistry/drugdiscovery/validation-libraries.html).

\section{Data Processing, Quality Control and Hit Scoring Criteria for HT Drug Screens}

All data processing and visualization was performed using custom programs written in the Pipeline Pilot platform (Accelrys, v.7.5) and the R program 6.6 [82-83]. The R drc package was used to fit sigmoidal curves [83]. ROC statistics were computed using the ROCR package [84].

The quality of the drug-screening studies against BCR-ABL ${ }^{\mathrm{WT}}$ and BCR$\mathrm{ABL}^{\mathrm{T} 315 \mathrm{I}}$ LICs was evaluated by multiple methods including, but not limited to, the identification of known antileukemic agents, identification of multiple compound replicates intentionally included in the 5600 collection, evaluation of control assay plates, and Z' (see Table 3.1) and other screening quality metrics. In the screening experiments, all assay plates demonstrated attenuation of dasatinib potency in the presence of IL7 or BCR-ABL mutation. An empirically determined, statistically significant but liberal cut-off of $>10 \%$ activity was chosen to include agents of relatively lower activity considering the high-risk nature of $\mathrm{Ph}+\mathrm{ALL}$, the drug-rich nature of the library as well as 
to allow for a more complete structure-activity relationship and therapeutic class analysis after subsequent potency determination through secondary screening.

The discriminatory power of the phenotypic assay was assessed using receiver operating characteristic (ROC) statistics. A total of 165 compounds were selected to sample the primary assay according to the distribution of observed activities. ROC curves were generated and area under curves (AUCs) calculated to determine the discriminatory power of the assay for BCR-ABL ${ }^{\mathrm{WT}}$ and $\mathrm{BCR}-\mathrm{ABL}^{\mathrm{T}}{ }^{15 \mathrm{I}} \mathrm{LICs}$, respectively.

The activity class designations were assigned based on the curve score and visual inspection of dose-response curves (see supplemental data in Appendix). The curve score is a 4 character code derived from the following heuristic:

- First character: letter (A,B,C or D) indicating curve quality

- $\mathrm{D}=$ less than 2 points above the noise (defined as outliers from the negative control population)

- $\mathrm{C}=\mathrm{r}^{2}<0.50$ or range $>200 \%$ or $\mathrm{EC}_{50}>$ maximum concentration tested

- $\mathrm{A}=\mathrm{r}^{2}>0.80$ and difference between two last points $<10 \%$ (indicating saturation)

- $\mathrm{B}=\operatorname{not} \mathrm{A}, \mathrm{C}$, or D

- N prefix = activity decreases with increase concentration

- Second character: number (1-6) indicating potency

- $1=\mathrm{EC}_{50}<0.1 \mu \mathrm{M}$

- $2=0.1 \mu \mathrm{M} \geq \mathrm{EC}_{50}<1.0 \mu \mathrm{M}$

- $3=1.0 \mu \mathrm{M} \geq \mathrm{EC}_{50}<10.0 \mu \mathrm{M}$

- $4=\mathrm{EC}_{50}>10.0 \mu \mathrm{M}$

- $5=$ all C and D curves are assigned 5

- Third character: number (1-6) indicating efficacy

- $1=75 \% \geq$ range $<200 \%$

- $2=50 \% \geq$ range $<75 \%$

- $3=25 \% \geq$ range $<50$

- $4=$ range $<25 \%$

- 5 = all C and D curves are assigned 5

- Fourth character: number (1-6) indicating hill slope

- $1=$ hill $\leq 2$

- $2=2<$ hill $\leq 4$

- $3=4<$ hill $\leq 8$

- $4=$ hill $>8$

- $5=$ all $\mathrm{C}$ and D curves are assigned 5

A complete list of all compounds evaluated in the secondary drug-screening experiments with their structure, supplier information, synonyms, $\mathrm{EC}_{50}$ values, and therapeutic classification is reported in the supplemental data in Appendix. 


\section{Cluster Analysis of Hits Using Therapeutic Classes}

The therapeutic cluster graph was generated by first assigning each compound an Anatomical Therapeutic Chemical Classification System (ATC). This classification system groups drugs into a 5 level hierarchy according to therapeutic indication and chemical properties. The first level indicates anatomical main group and consists of 1 letter; the second level indicates therapeutic main group and consists of 2 digits; the third level indicates therapeutic or pharmacological subgroup and consists of 1 letter; the fourth level indicates chemical, therapeutic or pharmacological subgroup and consists of one letter; and the fifth level indicates the chemical substance and consists of 2 digits. The resulting 7 characters constitute an ATC code, and a compound can be assigned multiple ATC codes. ATC codes can be depicted as a network graph by defining a node to represent each level in the ATC code and then connecting nodes according to parentchild relationships (e.g., first-level node A is the parent of second level nodes A01, A02, etc.). Compounds without an ATC code were assigned based on similarity to known agents, or were assigned to custom codes located under the parent V99 (labeled as "Not defined in ATC"). The frequency of ATC Level 1 and Level 2 codes among the 706 compounds submitted to dose-response analysis was determined.

The resulting network was visualized in Cytoscape (v 2.8.1) using the yFiles circular layout algorithm [85].

\section{Evaluation of Hits for Tolerance in Human Patients and Selectivity for MYC Gene Expression in Their Mechanism of Drug Action Using Public Databases}

Searches in Pubmed and MetaDrug ${ }^{\mathrm{TM}}$ software (by Genego, CA) [86] were performed for validated phenotypic hits to document approved use/uses in human disease/s if any, and known/predictive toxicity data in human and animal models, especially lymphotoxicity.Hits with favorable toxicity profiles were priortized using a previously described statistical analysis [87-88], similar to the COMPARE algorithm [8991], that evaluates National Cancer Institute (NCI) anti-cancer drug screening data. Briefly,the publically available databases containing half-maximal growth-inhibitory concentrations $\left(\mathrm{GI}_{50}\right)$ of approximately 4444 common chemicals and FDA-approved drugs against NCI60 panel of 60 human cancer cell-lines (of 9 different tissues of origin)

and complete gene expression datasets of all NCI60 cell lines, were accessed from the developmental Therapeutics Program (DTP) website (http://www.dtp.nci.nih.gov) and (http://discover.nci.nih.gov/cellminer/), respectively. A correlation analysis was performed between $\mathrm{GI}_{50}$ values and c-MYC expression across NCI60 cell lines for i) phenotypic hits demonstrating significant potency in a secondary screen, (whenever available in the DTP database, using NSC number as chemical identifier), ii) dasatinib, as a control for BCR-ABL-KI drug family, and iii) 4444 commonly used non-BCR-ABLKI DTP agents (to achieve statistical power and compute $\mathrm{p}$ values).

To extend the mechanistic understanding on DHA drug action against human cancer cells, a subset of DHA-senstive NCI60 cancer cell lines was identified. Using 
complete affymetrix data set from Cellminer ${ }^{\mathrm{TM}}$ website, the expression of each gene across the DHA-sensitive cell lines was subjected to correlative analyses with paired $\mathrm{GI}_{50}$ values of DHA. Pearson coefficient of correlation computed for individual genes was used to generate a rank order for all genes. A probeset of top 40 positively correlated genes was selected, representing a distinctive drug-sensitivity signature for DHA across human cancer cell lines. The expression level of this gene-set was then compared between murine LICs and primary Ph+ ALL leukemic samples, obtained from seven patients at the time of diagnosis (GSE17459, Pubmed). Cross-species correlative analysis was performed for each of the 40 genes by both expression values and rank order.

Gene expression signature of IL7-induced dasatinib resistance in LICs was compared with DHA senstivity signature across NCI60 panel to select overlapping genes which plausibly may play a role in overcoming dasatinib resistance.

\section{In Vivo Preclinical Therapeutic Studies}

For in vivo use, dasatinib (LC Labs, Woburn, MA) in citric acid ( $\mathrm{pH} 3.1$ ) [92], and DHA (Avachem, San Antonio, TX) in 0.5\% carboxy-methylcellulose $/ 0.5 \%$ Tween $80 / 0.5 \%$ benzyl alcohol, were administered by oral gavage. In toxicity studies (data not shown), DHA up to $300 \mathrm{mg} / \mathrm{kg}$ given as a single or split dose for $\leq 5$ days/week over a six week time period induced no significant weight loss, lethargy, seizures or deaths corroborating published findings [93]. During one or two agent therapeutic studies, animal weights were monitored daily ( 5 days/week) and drug dosing was adjusted accordingly.

\section{Bioluminescent Imaging to Track Therapeutic Responses In Vivo}

Bioluminescent imaging and analysis was performed using a Xenogen IVIS-200 system and Living Image software 3.01 (Caliper Life Sciences, Hopkinton, MA), as described previously [63]. Total bioluminescent flux measurements (photons/second) were quantified over the whole animal body. Recipient mice that remained clinically healthy 12 months after terminating therapy and had undetectable bioluminescent signal in vivo were designated "long-term survivors."

\section{Analysis of BCR-ABL KD Mutations}

Genomic DNA was extracted with phenol-chloroform from cultured cells, hematopoietic tissues, and leukemic-infiltrated meninges from moribund mice during therapy. Polymerase chain reactions (PCR) to selectively amplify only the human (and not murine) ABL KD were performed using primers as described previously $[63,79]$. Sequence analysis was performed from 10 to 20 independent bacterial colonies, while identified mutations were considered significant if present at $\geq 20 \%$ occurrence in individually sequenced clones. 


\section{CHAPTER 4. RESULTS}

\section{A BCR-ABL Kinase Domain Mutation Does Not Impart Any Advantage in Leukemia Progression In Vitro or In Vivo}

First, to model the clinically most dominant BCR-ABL mutation-dependent (molecular) resistance against all BCR-ABL-KI drugs in $\mathrm{Ph}+\mathrm{ALL}$, we generated $\mathrm{Arf}^{\prime-}$ pre-B cells driven with $\mathrm{p} 185 \mathrm{BCR}-\mathrm{ABL}^{\mathrm{WT}}$ or $\mathrm{p} 185 \mathrm{BCR}-\mathrm{ABL}^{\mathrm{T}}{ }^{\mathrm{A} 15 \mathrm{I}}$ mutant allele, referred as $A r f^{1-}$ BCR-ABL ${ }^{\mathrm{WT}}$ LICs and $A r f^{\prime-}$ BCR-ABL ${ }^{\mathrm{T} 315 \mathrm{I}}$ LICs, respectively. Both LICs performed similarly during growth assays in vitro (Figure 3.1) and displayed a potent and highly comparable leukemia-initiating capacity in vivo when injected in serial limiting cellular dilutions (Figure 4.1).

Thus, T315I BCR-ABL mutation, which imparts remarkable cell-intrinsic molecular drug-resistance, has no impact on leukemia progression in vivo in the absence of BCR-ABL-KI-treatment.

\section{Cytokine Screen Identifies IL7 as the Main Host Factor That Mediates Primary Dasatinib Resistance, Independent of BCR-ABL Mutations}

To address the role of cell extrinsic mechanisms in modulating primary responses to $\mathrm{BCR}-\mathrm{ABL}$ inhibitory therapy in $\mathrm{Ph}+\mathrm{ALL}$, fifteen cytokines normally present in the leukemia microenvironment were evaluated for their ability to protect murine $\mathrm{Arf}^{\prime-}$ BCR-ABL ${ }^{\text {WT }}$ LICs from dasatinib, the most potent clinical BCR-ABL inhibiting agent (Figure 4.2a). $\mathrm{Arf}^{\prime-} \mathrm{BCR}-\mathrm{ABL}^{\mathrm{WT}}$ LICs were confirmed beforehand to not harbor any drug-resistant mutations in the $A B L \mathrm{KD}$. It is important to note that genetically-defined $\mathrm{Arf}^{\prime-} \mathrm{BCR}-\mathrm{ABL}{ }^{\mathrm{WT}}$ LICs are transformed primary pre-B cells that retain phenotypic and signaling properties essential for suspended leukemic blasts to respond to the surrounding host-environment [48]. These properties are typically not present in established leukemic cell lines that have been extensively cultured in vitro.

Among the cytokines tested, low-level $(<1 \mathrm{ng} / \mathrm{mL})$ IL7 provided the most potent and robust protection against dasatinib-induced growth arrest and killing of $\mathrm{Arf}^{\prime-}$ BCR-ABL ${ }^{\mathrm{WT}}$ LICs (Figure 4.2a). In addition, Interleukin3 (IL3) $[62,69,94]$, thymic stromal lymphopoietin (TSLP, an IL7-like cytokine that shares a receptor subunit with IL7) [95] and Interleukin4 (IL4) conferred resistance, but much more weakly. In contrast, TGF $\beta$ enhanced dasatinib activity.

\section{IL7 Mediates Primary Drug Resistance against All Approved BCR-ABL-KIs in Murine and Human Ph+ ALL Cells, Which Is Completely Reversible}

Next, we extended the evaluation of IL7, our most potent candidate from the cytokine screen, to assess its impact on the performance of other drugs, including 

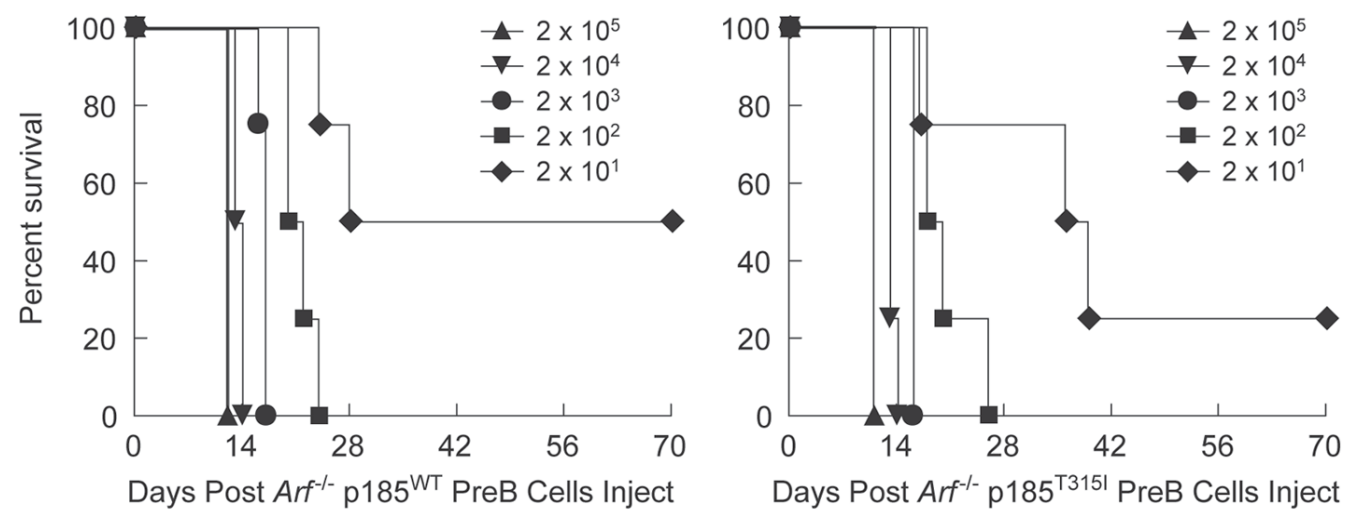

Figure 4.1. Leukemia stem cell (LSC) function assay by serial dilution and implantation comparing the in vivo leukemia-initiating capacity of $\mathrm{Arf}^{-1-} \mathrm{p} 185^{\mathrm{WT}}$ (left) and $\mathrm{Arf}^{--} \mathrm{p}^{185^{\mathrm{T} 315 \mathrm{I}}}$ pre-B (right) populations.

Arf $^{\prime-}$ p185-transformed pre-B murine cells are referred as LICs. Kaplan-Meier curves represent the overall survival of nonconditioned immunocompetent $\mathrm{C} 57 \mathrm{Bl} / 6 \mathrm{~J}$ recipient mice that received serial log-fold dilutions ( $\mathrm{n}=4$ per arm) of LIC-number (as indicated in the inset legend) on day 0 . 

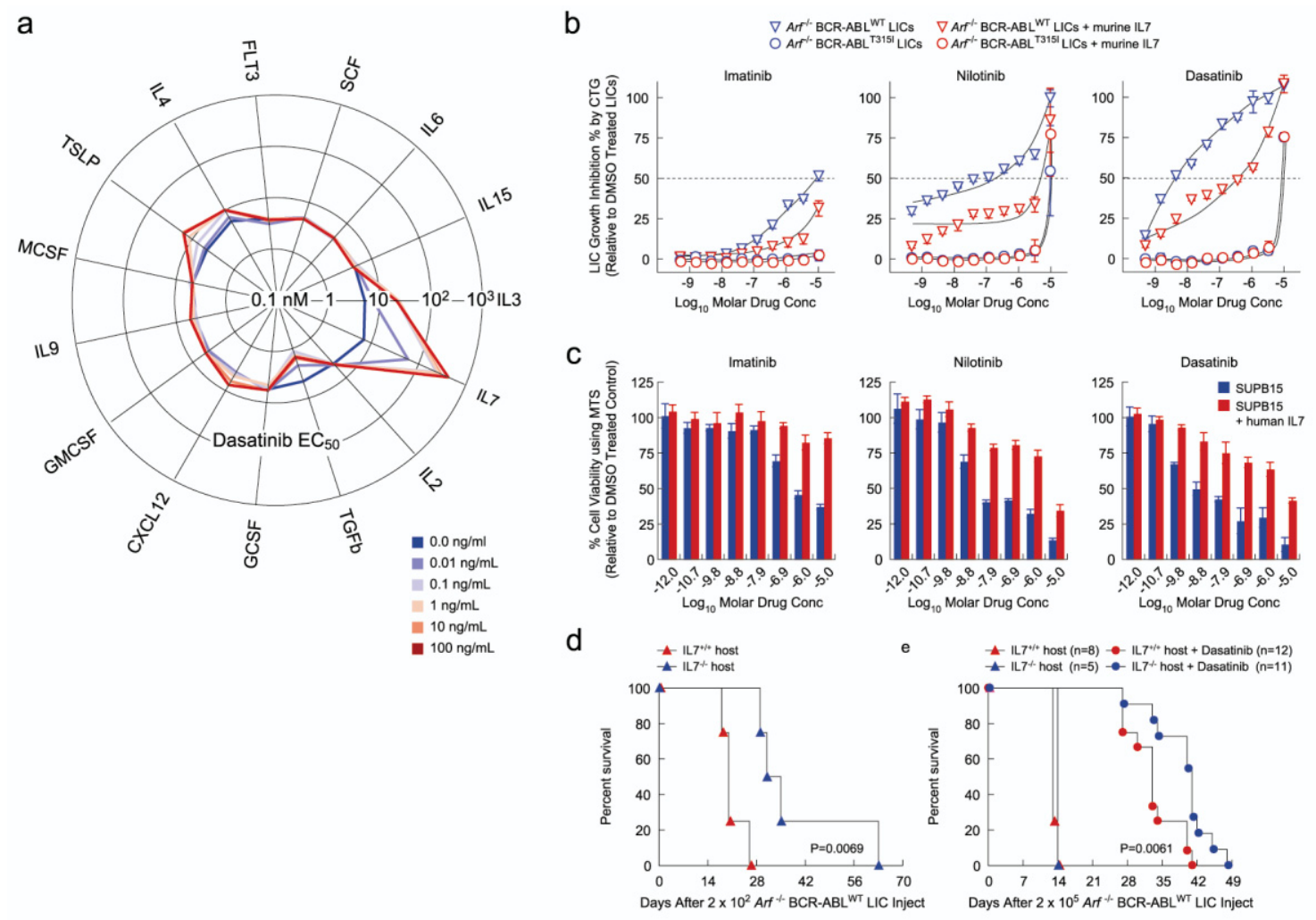

\section{Figure 4.2. IL7 confers BCR-ABL ${ }^{\text {MUTANT }}$-independent primary drug-resistance against BCR-ABL-KIs in Ph+ ALL.}

(a) Radar plot representing the effect of 15 hematopoietic cytokines on dasatinib potency $\left(\mathrm{EC}_{50}\right)$ against $\mathrm{Arf}^{-1}$ BCR-ABL ${ }^{\mathrm{WT}}$ LICs. CTG-assay measured LIC killing after $72 \mathrm{~h}$ dasatinib exposures in presence of increasing concentrations of indicated cytokines. Each spoke represents an individual cytokine; each line graph represents dasatinib $\mathrm{EC}_{50}(\mathrm{nM})$ at indicated titration of the parent cytokine (see supplemental data in Appendix). (b) $\mathrm{Arf}^{\prime-}$ BCR-ABL ${ }^{\mathrm{WT}}$ (triangles) and $\mathrm{Arf}^{\prime-} \mathrm{BCR}-\mathrm{ABL}^{\mathrm{T} 315 \mathrm{I}}$ (circles) LICs were treated with drug or DMSO for $72 \mathrm{~h}$ in the absence (blue) or presence (red) of $1 \mathrm{ng} / \mathrm{mL} \mathrm{mIL7}$, in triplicates. For each drug concentration, LIC growth measured by CTG-assay was normalized to DMSO-treated LICs and fit using nonlinear regression $(+/-s d)$.

(c) Viability of human Ph+ ALL SUP-B15 cells after $72 \mathrm{~h}$ drug-treatment in absence or presence of $25 \mathrm{ng} / \mathrm{mL}$ hIL7, was measured by MTS assay. Values were normalized to DMSO-treated cells. (d, e) Kaplan-Meier survival curves showing overall survival in $\mathrm{IL}^{+/+}$and $\mathrm{IL7}^{-/-}$B16 host-mice receiving day 0 tail vein injection of (d) $2 \times 10^{2} \mathrm{Arf}^{/-}$ BCR-ABL ${ }^{\text {WT }}$ LICs (vehicle treated), with median survival of 20 days in $\operatorname{IL}^{+/+}(\mathrm{n}=4)$ versus 33 days in $\mathrm{IL}^{-/}$mice $(\mathrm{n}=4), \mathrm{P}=0.0069$, or (e) $2 \times 10^{5} \mathrm{Arf}^{--} \mathrm{BCR}-\mathrm{ABL}{ }^{\mathrm{WT}} \mathrm{LICs}$. Data are an average of 2 independent experiments in which vehicle or dasatinib therapy was initiated on day 7 or 9. Survivals of vehicle-treated $\operatorname{IL~} 7^{+/+}$and $\operatorname{IL} 7^{-/-}$mice were comparable. Dasatinib treatment caused a median survival of 33 days in $\operatorname{IL} 7^{+/+}(n=12)$ versus 41 days in $\mathrm{IL}^{-/-}(\mathrm{n}=11)$ mice, $\mathrm{P}=0.0061$. 
BCR-ABL-KIs and non-BCR-ABL inhibiting drugs. IL7 significantly attenuated the potency of all FDA-approved BCR-ABL-KIs against murine $\mathrm{Arf}^{/-} \mathrm{BCR}^{-\mathrm{ABL}}{ }^{\mathrm{WT}} \mathrm{LICs}$ (Figure 4.2b) and the human $\mathrm{Ph}+\mathrm{ALL}$ cell line SUP-B15 (Figure 4.2c). In contrast, IL7 did not affect the potency of tested non-BCR-ABL drugs underscoring the specificity of this cytokine protection (Figure 4.3).

The specificity and dynamic nature of IL7 in mediating primary resistance against BCR-ABL-targeting drugs was further confirmed by four in vitro findings. First, mechanistic evaluations by FACS analysis revealed dasatinib-induced phenotypic effects of $\mathrm{G}_{0}-\mathrm{G}_{1}$ growth arrest and apoptosis of $\mathrm{Arf}^{/-}$BCR-ABL ${ }^{\mathrm{WT}}$ LICs were significantly blunted by the presence of cell-extrinsic IL7 during drug exposure (Figure 4.4a and b). Second, transient pharmacological BCR-ABL-inhibition, achieved by washing out drug/cytokine after a period of dasatinib/IL7 co-treatment (see 'Drug/cytokine washout and cytokine recovery studies' in Chapter 3), dramatically increased the responsiveness of $\mathrm{Arf}^{--}$BCR-ABL ${ }^{\mathrm{WT}}$ LICs to cell-extrinsic IL7 (Figure 4.4a, c and d) [96]. Third, removing (washing out) IL7 completely reversed cytokine protection and restored dasatinib's potent activity to kill $\mathrm{Arf}^{/-}$BCR-ABL ${ }^{\mathrm{WT}} \mathrm{LICs}$ (see 'dasatinib re-challenge studies' in Chapter 3, data not shown). Fourth, IL7 significantly rescued the clonal outgrowth capacity of single flow-sorted $\mathrm{Arf}^{/-} \mathrm{BCR}^{-\mathrm{ABL}}{ }^{\mathrm{WT}}$ LICs during prolonged treatments with dasatinib (Figure 4.5).

Thus, IL7 imparts primary refractoriness against approved BCR-ABL-KIs in murine and human $\mathrm{Ph}+\mathrm{ALL}$ cells, which exists as a completely reversible phenomenon.

\section{Physiological Levels of Host-expressed IL7 Contributes to an Aggressive, Dasatinib- resistant Phenotype of Ph+ ALL In Vivo}

We studied the effect of physiologic levels of IL7 on leukemia progression and dasatinib responsiveness in vivo. Host IL $7^{+/+}$(i.e. wild-type; WT) mice injected with $2 \times 10^{2} \mathrm{Arf}^{/-}$BCR-ABL ${ }^{\mathrm{WT}}$ LICs showed more rapid leukemia onset compared to strainmatched IL $7^{-/}$(null) hosts (Figure 4.2d), suggesting that murine $\mathrm{Ph}+\mathrm{ALL}$ responds to host-IL7 with accelerated progression. Injecting $2 \times 10^{5} \mathrm{Arf}^{/-} \mathrm{BCR}-\mathrm{ABL}{ }^{\mathrm{WT}} \mathrm{LICs}$ overcame this trend (Figure 4.2e), suggesting the phenomena is most important during the phase of disease establishment. Host-expressed physiologic level of IL 7 significantly shortened the survival benefit from dasatinib monotherapy of BCR-ABL ${ }^{\mathrm{WT}}$ leukemias (Figure 4.2e). This finding is the first definitive demonstration of a single host-cytokine in inducing BCR-ABL-KI drug resistance in vivo. Of note, terminal clinical presentation in IL7-WT host-mice was either with signs of CNS disease (bulging skull, uneven gait, erratic behavior) or frank relapse in rest of the body, whereas the majority of IL $7^{-/}$hostmice presented with signs of CNS disease necessitating their sacrifice, consistent with reports on low bioavailability of BCR-ABL-KIs in CNS [70-71].

From a therapeutic perspective, these findings in combination with our in vitro observations support that in vivo responses to dasatinib therapy in $\mathrm{Ph}+\mathrm{ALL}$ are modulated by cell-extrinsic IL7 in the absence of BCR-ABL KD mutations. 

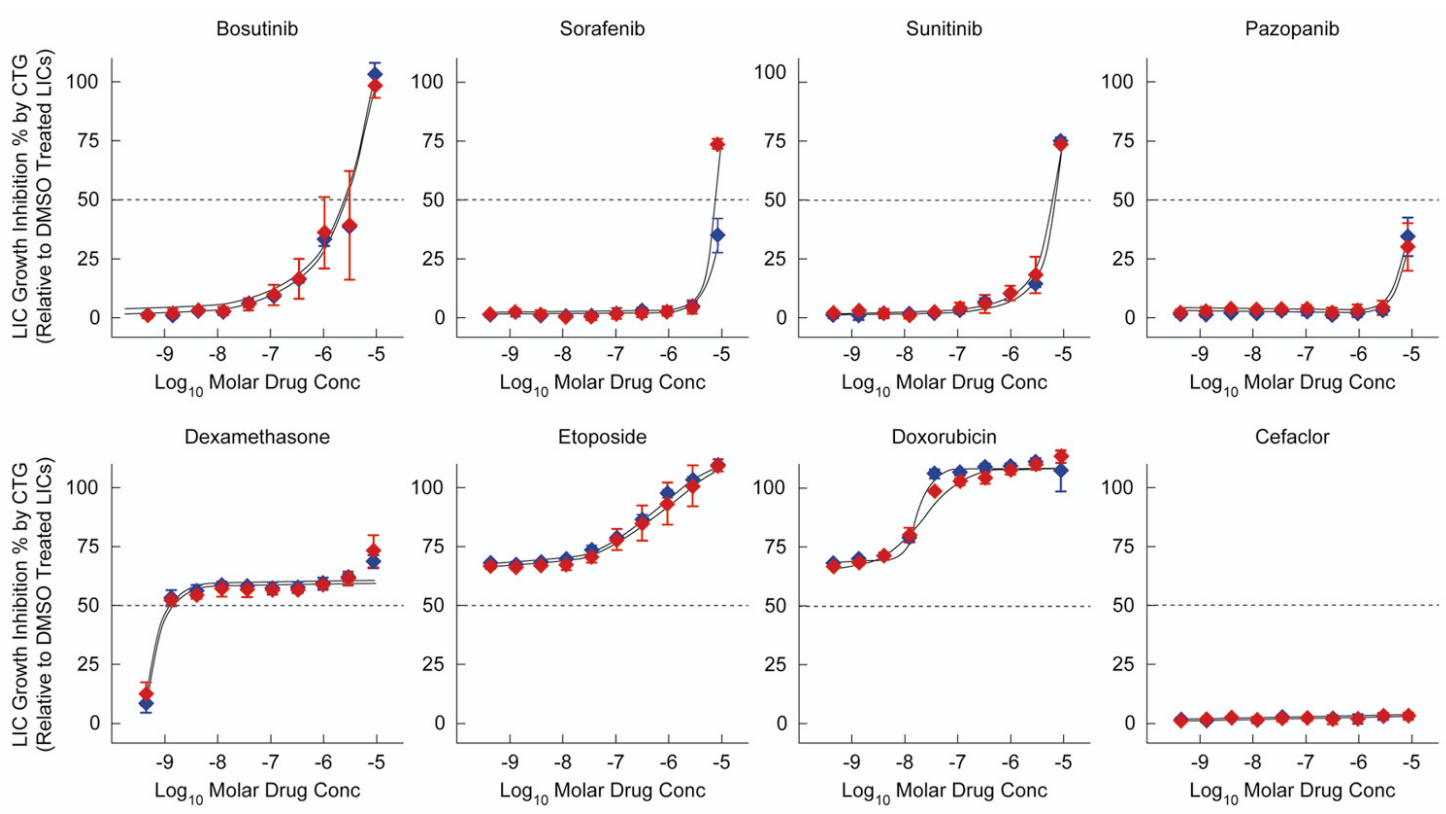

- Arf ${ }^{-1-}$ BCR-ABL ${ }^{W T}$ LICs

- rrf $^{\text {- }}$ BCR-ABL ${ }^{W T} \mathrm{LICs}+$ murine IL7

Figure 4.3. Comparative evaluation of IL7-mediated protection conferred against non-BCR-ABL-specific targeting drugs (top panel) and conventional cytotoxic antileukemic clinical drugs (bottom panel).

Arf $^{/-}$BCR-ABL ${ }^{\text {WT }}$ LICs were treated with indicated drugs or DMSO (no drug) for $72 \mathrm{~h}$ in the absence (blue) or presence (red) of high $(10 \mathrm{ng} / \mathrm{mL})$ murine IL 7, in triplicates. The antibiotic drug Cefaclor (often used in leukemia clinics) was used as a non-antileukemic drug control. For each drug concentration, LIC growth was measured by the CTG-assay and average values were normalized to DMSO-treated LICs and graphed $(+/-\mathrm{sd})$. 
a
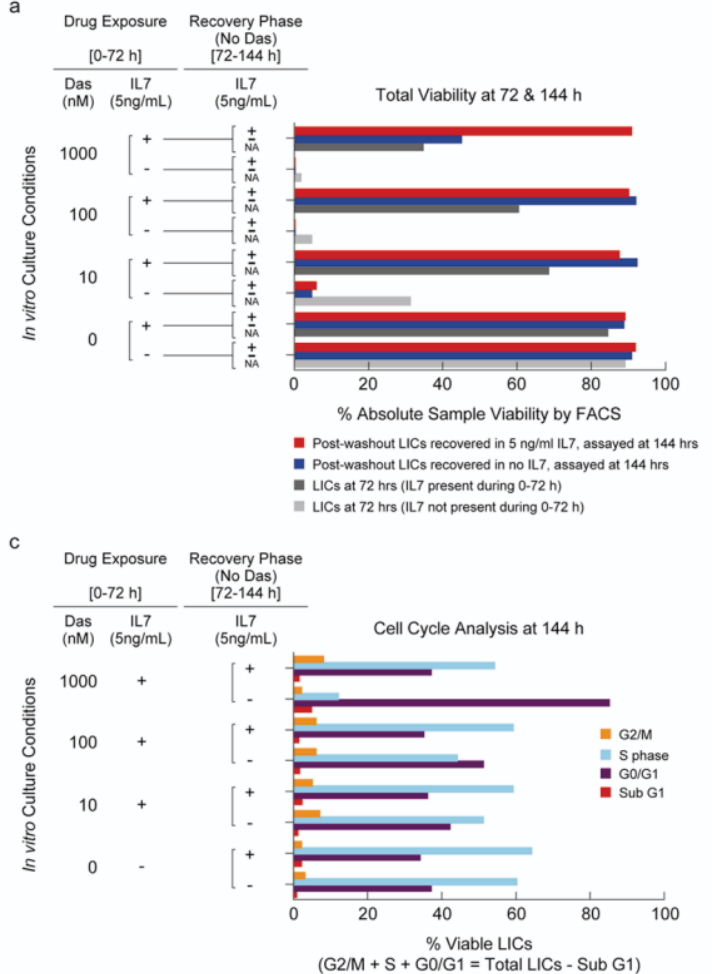

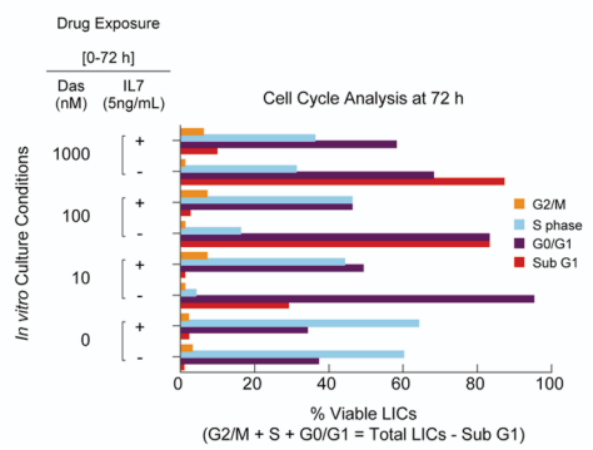

d Drug Exposure Recovery Phase

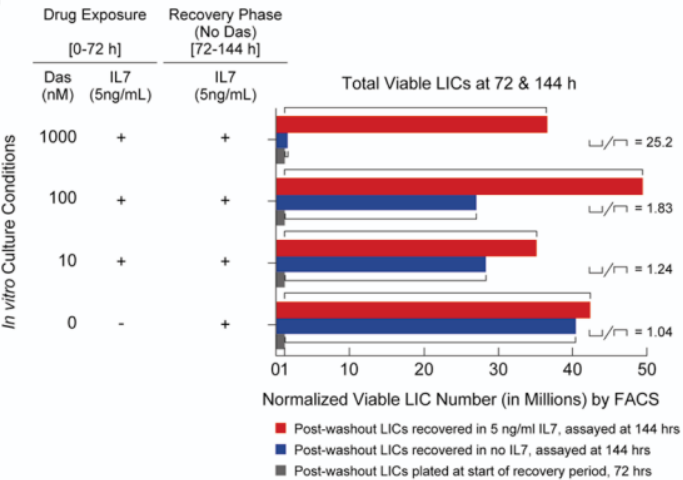

Figure 4.4. Protection of $\boldsymbol{A r f}^{-1-}$ BCR-ABL ${ }^{\text {WT }}$ LICs by IL7 during dasatinib exposures ( $(0$ to $72 \mathrm{~h}$ ) and in the post-exposure recovery period (72 to $144 \mathrm{~h}$, no dasatinib).

See Chapter 3 for details. (a) Total sample viabilities (DAPI staining, FACS) at the end of exposure period (dark gray=IL7; light gray=No IL7) and recovery period (red=IL7; blue=No IL7). Light gray versus dark gray comparison depicts significant IL7 protection during dasatinib exposures. Few LICs surviving dasatinib exposures in the absence of IL7 (light gray), later deteriorated and died during recovery period, irrespective of IL7, confirming that BCR-ABL inhibitor exposures commit BCR-ABL+ cells to death [56]. IL7 significantly improved recovery of dasatinib/IL7 co-treated LICs (red vs blue), clearly evident at $1000 \mathrm{nM}$ but not at $100 \mathrm{nM}$ and $10 \mathrm{nM}$ dasatinib concentration because of over-confluent growth in presence of IL7 (see (c) and (d)). (b) Cell cycle changes (FACS) at the end of exposure period (corresponding to light and gray bars in (a)). Incremental dasatinib doses (which cause incremental BCR-ABL inhibition [55]) induced cell cycle arrest and apoptosis; IL7 significantly protected LICs from these changes (c) Cell cycle analysis at the end of recovery period (corresponding to blue and red in (a)). (d) Conditions showing positive LIC growth during recovery period, normalized for equal number of LICs plated at start of recovery (dark grey). Ratio of fold LIC-change in presence (face-down bracket) to that in absence (face-up bracket) of IL 7 increased in LICs with higher previous BCR-ABL inhibition from higher dasatinib concentrations during exposure period [96]. 


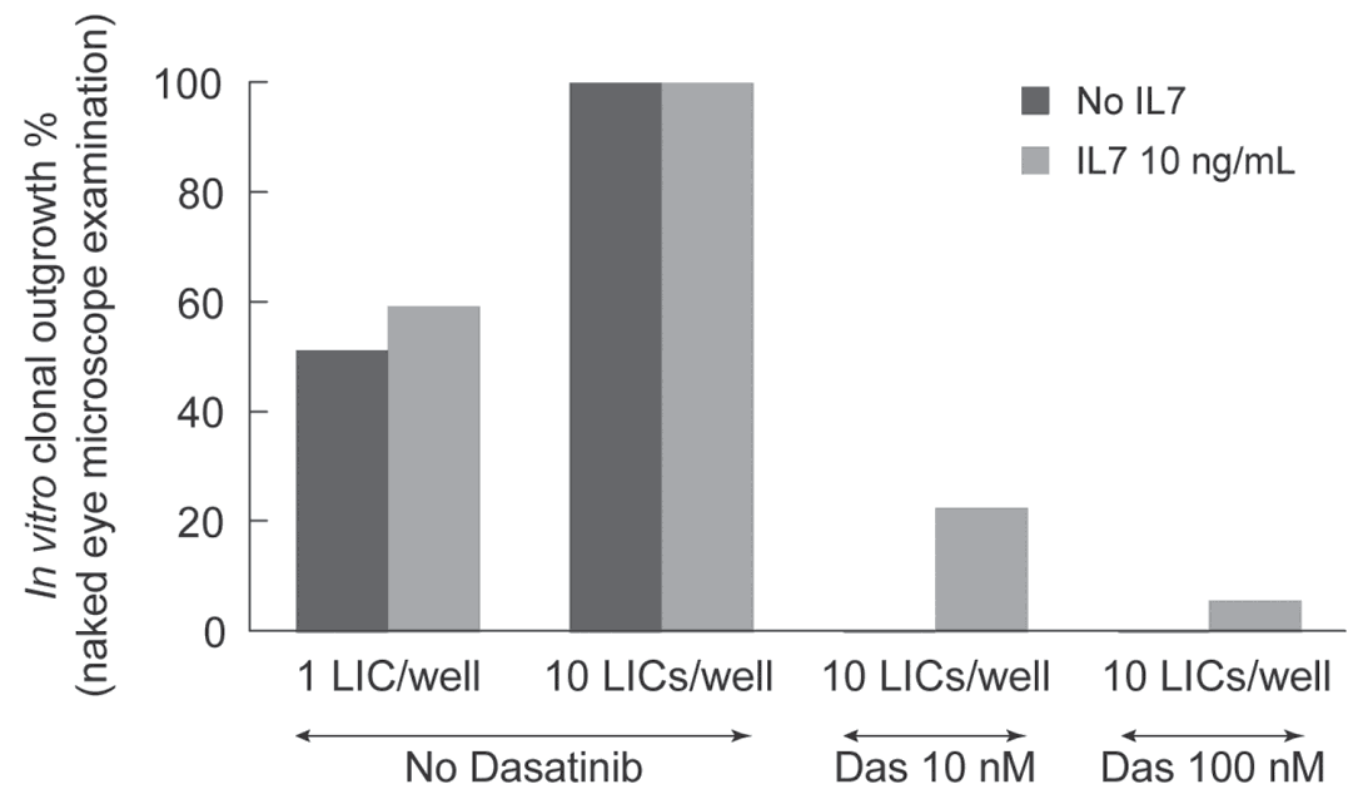

Figure 4.5. In vitro leukemia clonal outgrowth efficiency assays.

Either 1 or 10 viable $\mathrm{Arf}^{/-}$BCR-ABL ${ }^{\mathrm{WT}}$ LICs per well sorted by single-cell flow cytometry into 3 clear-bottom 384-well plates for each condition were treated $(\mathrm{t}=0)$ with DMSO ( $0.1 \%$ by volume) or dasatinib in the absence (dark grey) or presence (light grey) of IL7 ( $5 \mathrm{ng} / \mathrm{mL})$. After 8 days of incubation, naked-eye microscopic examination was performed. Wells showing significant positive growth (approximately $\geq 50$ LICs per well) were scored positive and percentage of positive wells for each condition indicated on the $\mathrm{x}$-axis was calculated. 


\section{IL7 Protects against Dasatinib by Strongly Counteracting the Decrease in pSTAT5 Protein and c-MYC Expression}

What intra-cellular changes initiated by the host-IL7 are critical for imparting primary dasatinib resistance? Arf ${ }^{/-}$BCR-ABL ${ }^{\mathrm{WT}}$ LICs were briefly (4 h) challenged with $100 \mathrm{nM}$ dasatinib in the presence or absence of IL7 and then evaluated using a cell-signaling kit (Luminex ${ }^{\circledR} 8$-plex kit by Millipore, MA) which detects level of phosphorylated (active) form of eight different cell-signaling proteins relevant to cancer biology (Figure 4.6a) and gene expression arrays (Figure 4.6b). Comparative assessments in the global gene expression and various signaling pathways allowed us to detect the intra-cellular changes crucial for mediating IL7-induced dasatinibrefractoriness.

Among the eight phosphorylated signaling proteins evaluated, short dasatinib exposure in the absence of IL7 decreased the level of mainly pSTAT5 protein (Figure 4.6a). STAT5 is an established phosphorylation target of uninhibited BCR-ABL kinase activity [97-98], and decrease in the level of pSTAT5 is commonly used as a surrogate marker of cellular BCR-ABL-inhibition [99-100]. Interestingly, the presence of IL7 significantly maintained high levels of pSTAT5 in Arf $^{/-}$BCR-ABL ${ }^{\mathrm{WT}}$ LICs despite pharmacological BCR-ABL inhibition by dasatinib (Figure 4.6a) [101]. These findings were confirmed using conventional western blotting (Singh and Opferman, unpublished). A positive trend was observed between the levels of pSTAT5 protein in $\mathrm{Arf}^{/-}$ BCR-ABL ${ }^{\mathrm{WT}}$ LIC samples at 4 hours and their respective total sample viability and total viable cell counts after 72 hours, as measured by CTG and FACS (compare with Figure 4.2b and Figure 4.4a, respectively). This suggested a new mechanistic role for pSTAT5 in IL7 mediated rescue of Ph+ ALL from BCR-ABL inhibitory therapy.

From gene expression data, we dissected a set of mRNAs that underwent the most significant quantitative change in cellular expression due to brief dasatinib exposure in the absence of IL7, relative to untreated LICs, to determine a 'dasatinib response signature' in $\mathrm{Ph}+\mathrm{ALL}$. Using this signature as a reference, we identified a subset of mRNAs which were most significantly protected from dasatinib-induced change in cellular expression due to the presence of IL7 during drug exposure, referred as 'IL7-imparted dasatinib resistance signature.' Enrichment analysis (Figure 4.6b) of the cytokine-mediated dasatinib resistance signature demonstrated that IL7 strongly protects cellular expression of c-MYC and c-MYC target genes, with c-MYC being the second highest protected mRNA by IL7 (see supplemental data in Appendix).

Cellular binding of growth factor IL7 activates the receptor-associated JAK3-STAT5 signaling pathway [101], resulting in the direct upregulation of c-MYC by activated STAT5 (pSTAT5) [102]. Thus, IL7 counteracts the decrease in both pSTAT5 and c-MYC resulting from pharmacological inhibition of BCR-ABL by dasatinib, and recues Arf $^{/-}$BCR-ABL WT LICs by initiating IL7-JAK3-pSTAT5-cMYC signaling. 
a

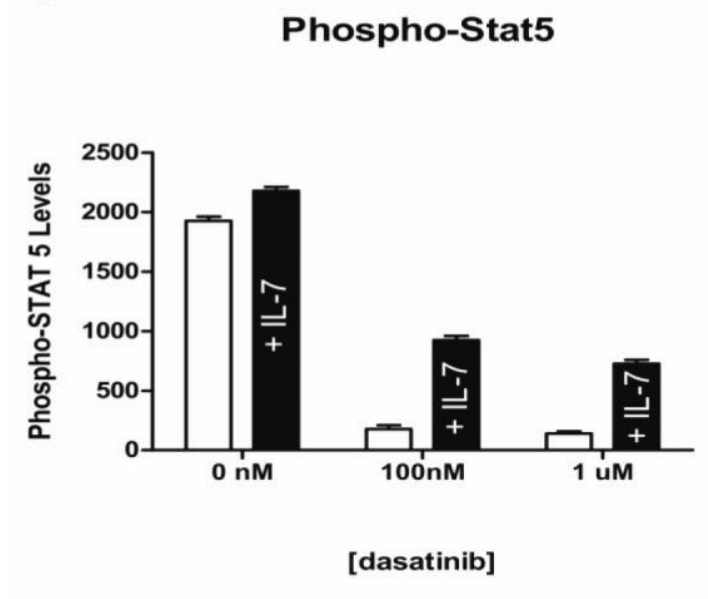

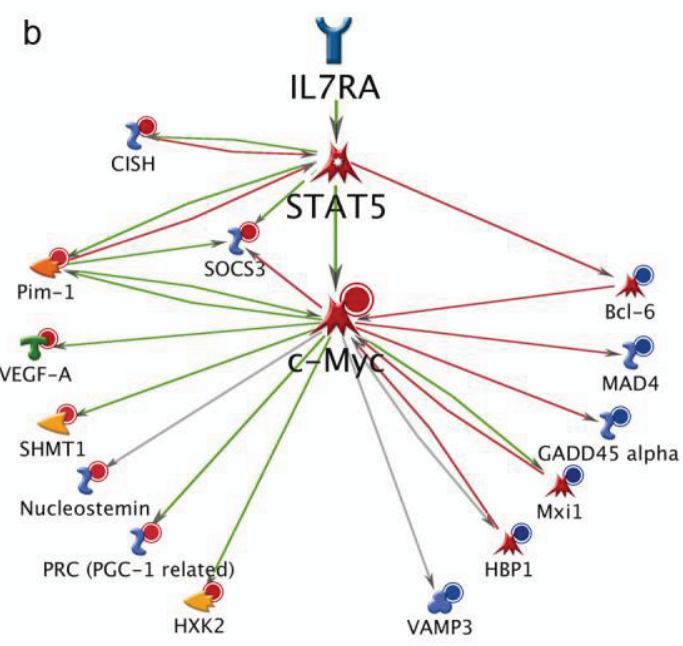

Figure 4.6. Mechanistic evaluation of host-IL7 imparted primary resistance against dasatinib.

Arf $^{/-}$BCR-ABL ${ }^{\mathrm{WT}}$ LICs were challenged with dasatinib or DMSO for 4 hrs in presence or absence of IL7. (a) Levels of pSTAT5 measured at 4 hrs by Luminex 8-plex multipathway kit are presented. Change in level of pErk/MAP kinase 1/2, pSTAT3, pJNK, p70 S6 kinase, IkBalpha, CREB, and p38 was statistically insignificant and is not shown. (b) Cartoon shows enrichment analysis of 'IL7 protection gene signature against dasatinib'. Increase or decrease in expression of genes by dasatinib alone (no IL7) is presented by red (decrease) and blue (increase) color, respectively. Size of the circle presents fold-protection against dasatinib-induced change in gene expression due to the presence of IL7.Only top representative genes undergoing significant change in the presence of IL7 are shown. See supplemental data in Appendix for complete data set. 


\section{High pSTAT5 and IKAROS Haploinsufficiency Are Candidate Resistance Biomarkers with c-MYC as a Convergence Node of Resistance against BCR-ABL Inhibitor Therapy in Ph+ ALL}

Our mechanistic studies implicated c-MYC expression in cytokine mediated BCR-ABL-KI-resistance. Because IKAROS directly represses c-MYC expression in pre-B cells and IKAROS haploinsufficiency relieves such c-MYC repression [103], we hypothesized that IKAROS haploinsufficiency by derepressing c-MYC expression may also impart cell-intrinsic BCR-ABL-KI-resistance in $\mathrm{Ph}+$ pre-B cells.

To confirm the role of pSTAT5, c-MYC and IKAROS haploinsufficiency in ABL KD mutation-independent drug-resistance, we utilized fluorescent vectors expressing constitutively active STAT5 (CA-STAT5) single or double mutants, human c-MYC or dominant negative (DN) IKAROS (IK6), imitating constitutive signaling by pSTAT5, c-MYC-overexpression and IKAROS haploinsufficiency, respectively (Figure 4.7). Analogus to IKAROS-WT, the IK1 mutant binds to the c-MYC promoter and inhibits its expression, whereas the IK6 mutant derepresses c-MYC expression [75, 103]. Arf $^{\prime-}$ BCR-ABL ${ }^{\mathrm{WT}}$ LICs transduced (florescent) with these vectors were mixed with parent non-transduced (non-florescent) LICs and subjected to drug-competition assays in the absence of IL7. HT-flow assay (please refer Figure 3.3) performed on day 2, 3, 5 and 9 relative to day 0 allowed a temporal assessment of 'engineered' cell-intrinsic genetic changes on growth and cellular response to dasatinib and imatinib.

In the absence of BCR-ABL-KI treatment (Figure 4.7a), proliferation of $\mathrm{Arf}^{/-}$ BCR-ABL ${ }^{\text {WT }}$ LICs expressing GFP-only ('empty' vector), IK6 or c-MYC was comparable to non-transduced parent LICs, whereas fluorescent $\mathrm{Arf}^{/-}$BCR-ABL ${ }^{\mathrm{WT}} \mathrm{LICs}$ expressing pre-validated single or double CA-STAT5 mutants or the IK1 mutant [75, 103], were quickly lost in culture. The later observation corroborated the known essential role of c-MYC expression in sustaining Ph+ LICs [60-61].

Treatment (Figure 4.7b) of control LIC mixtures with increasing imatinib and dasatinib concentrations did not alter the ratio between non-transduced LICs and daughter LICs transduced with GFP-only vectors. The total mixture viability (by DAPI exclusion) of control LIC mixtures decreased in a dose and time proportionate fashion. This corroborated our previous flow-cytometry results (Figure 4.4a), and confirmed the overall quality of HT-flow drug-screening runs. In stark contrast to control LIC mixtures, drug exposures temporally selected the $\mathrm{Arf}^{/-} \mathrm{BCR}^{-\mathrm{ABL}}{ }^{\mathrm{WT}}$ LICs transduced with vectors expressing c-MYC or IK6 or single mutant CA-STAT5 or double mutant CA-STAT5. Remarkably, HT-flow counter genetic-screen demonstrated that the degree of LICselection to be quantitatively proportional to BCR-ABL-inhibition achieved by incremental BCR-ABL-KI doses (Figure 4.7b and c) [55]. These results were later confirmed with western blots (unpublished, Singh and Opferman). Thus, pSTAT5, c-MYC and functional-loss of IKAROS, all mediate BCR-ABL mutation-independent drug resistance according to proposed model in Figure 4.7d. In a direct test of c-MYC as a node for dasatinib-resistance in $\mathrm{Ph}+\mathrm{ALL}$, dasatinib-challenges were performed against Arf $^{/-}$BCR-ABL ${ }^{\mathrm{WT}}$ LICs expressing either an empty vector or c-MYC protein. 
Figure 4.7. Results of counter genetic screen by HT-flow confirming the role of pSTAT5 activity, IKAROS haploinsufficiency and c-MYC over-expression in primary drug resistance against BCR-ABL-inhibiting drugs.

Mixtures composed of non-transduced (non-fluorescent) parent $\mathrm{p} 185^{\mathrm{WT}} \mathrm{LICs}$ and transduced (fluorescent) daughter $\mathrm{p} 185^{\mathrm{WT}}$ LICs expressing protein of interest (as per legends) were exposed to (a) no drug (controls) or (b,c) wide-range of imatinib and dasatinib concentrations in the absence of IL 7, in triplicate wells. Percentage of fluorescent (transduced) LICs on day 0, 2, 5 and 9 was measured by HT-flow assay. (b) Ratio of average $(n=3)$ number of viable fluorescent (transduced) LICs (by DAPI) on day 2, 5 and 9 relative to day 0 , at each indicated experimental condition, are shown for dasatinib and imatinib. Change in ratio is presented using a three-color graded scale ( red $>1$, yellow $=1$, green $<1$; dark (red/green) color implies higher change and light (red/green) color implies lower change. (c) X-Y line graph representing temporal selection (x-axis) of CA-STAT5 double mutant (DM) expressing LICs (relative to day=0) due to exposure to dasatinib concentrations, in a dose-response fashion. (d) Proposed mechanistic model of BCR-ABL mutation-independent drug-resistance against BCRABL inhibiting drugs in Ph+ ALL.

* Zero values (darkest green) indicate that total sample viability is below the detectionthreshold by flow cytometry.

** Relatively low number of LICs transduced by single and double CA-STAT5 mutants on day $=0$ in (a) due to low transduction efficiency (from day -2 to 0 ) later allowed much higher fold selection within the same wells (from day 0 to 9) in (b). 


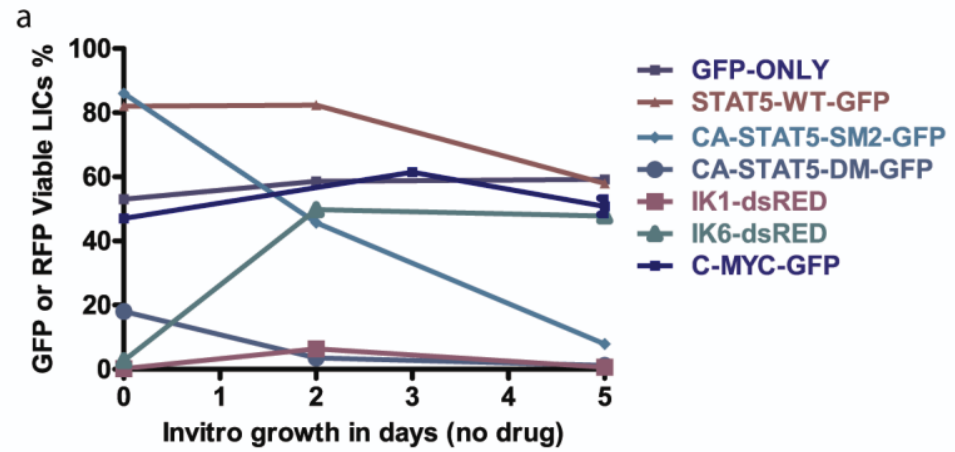

\begin{tabular}{|c|c|c|c|c|c|c|c|c|c|c|c|c|c|c|}
\hline \multirow[t]{2}{*}{ b } & & \multicolumn{7}{|c|}{ DASATINIB (nM) } & \multicolumn{6}{|c|}{ IMATINIB (nM) } \\
\hline & DAY & 0.03 & 0.1 & 0.3 & 1 & 3 & 10 & 100 & 30 & 100 & 300 & 1000 & 3000 & 10000 \\
\hline \multirow[t]{2}{*}{ GFP } & 2 & 1.023 & 1.015 & 0.994 & 0.975 & 0.996 & 0.929 & 0.000 & 1.020 & 1.046 & 1.044 & 1.001 & 0.981 & 0.827 \\
\hline & 5 & 1.034 & 1.036 & 1.029 & 0.916 & 0.684 & 0.000 & 0.000 & 0.885 & 0.976 & 1.080 & 1.114 & 0.884 & 0.000 \\
\hline \multirow[t]{3}{*}{ STAT5-WT } & 2 & 1.089 & 1.106 & 1.042 & 1.033 & 0.966 & 1.026 & 0.000 & 0.998 & 1.023 & 1.087 & 1.100 & 1.003 & 0.986 \\
\hline & 5 & 1.332 & 1.362 & 1.655 & 1.606 & 1.334 & 0.000 & 0.000 & 0.956 & 1.130 & 1.396 & 1.680 & 1.514 & 0.000 \\
\hline & 9 & 1.185 & 1.094 & 1.658 & 1.579 & 0.000 & 0.000 & 0.000 & 1.000 & 0.994 & 1.110 & 1.219 & 1.546 & 0.000 \\
\hline \multirow{2}{*}{ CA-STAT5SM1 } & 5 & 3.812 & 4.659 & 7.136 & 6.781 & 5.978 & 0.000 & 0.000 & 0.648 & 1.288 & 3.008 & 6.723 & 6.892 & 0.000 \\
\hline & 9 & 6.157 & 5.858 & 12.807 & 13.056 & 14.756 & 0.000 & 0.000 & 1.000 & 1.487 & 3.056 & 7.218 & 12.807 & 0.000 \\
\hline \multirow[t]{3}{*}{ CA-STAT5SM2 } & 2 & 1.543 & 1.632 & 1.535 & 1.429 & 1.113 & 1.007 & 0.000 & 1.057 & 1.243 & 1.409 & 1.709 & 1.415 & 1.058 \\
\hline & 5 & 5.749 & 6.834 & 11.451 & 10.791 & 11.740 & 0.000 & 0.000 & 0.668 & 1.685 & 5.353 & 10.881 & 9.391 & 0.000 \\
\hline & 9 & 9.802 & 17.440 & 22.033 & 26.813 & 31.692 & 0.000 & 0.000 & 1.560 & 2.989 & 13.604 & 27.681 & 0.000 & 0.000 \\
\hline CA-STAT5DM & 2 & 1.811 & 1.925 & 2.566 & 2.472 & 1.877 & 1.745 & 0.000 & 1.038 & 1.132 & 1.679 & 2.840 & 2.509 & 1.745 \\
\hline IK6 & 9 & 1.553 & 1.579 & 3.461 & 3.095 & 0.000 & 0.000 & 0.000 & 0.944 & 1.027 & 1.640 & 3.153 & 0.000 & 0.000 \\
\hline \multirow[t]{3}{*}{ MYC } & 3 & 1.309 & 1.335 & 1.515 & 1.422 & 0.866 & 0.560 & 0.000 & 1.010 & 1.081 & 1.302 & 1.574 & 1.082 & 0.150 \\
\hline & 5 & 1.489 & 1.530 & 1.877 & 1.778 & 1.193 & 0.000 & 0.000 & 0.965 & 1.102 & 1.530 & 1.843 & 1.810 & 0.000 \\
\hline & 9 & 1.633 & 1.480 & 2.645 & 2.818 & 2.964 & 0.000 & 0.000 & 1.048 & 1.227 & 1.626 & 2.063 & 3.075 & 0.000 \\
\hline
\end{tabular}

C

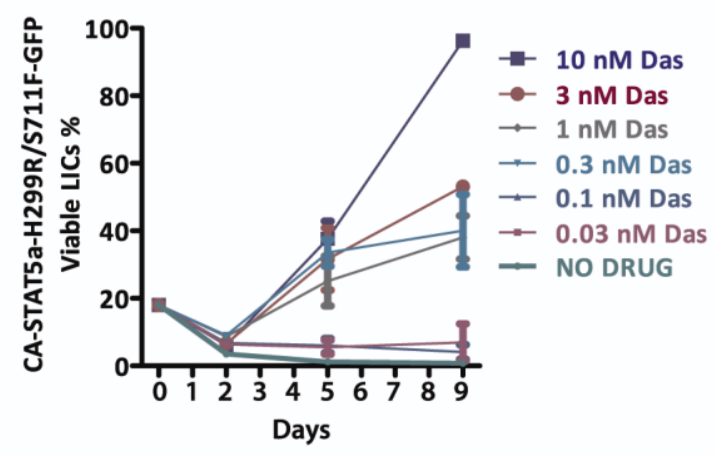

d

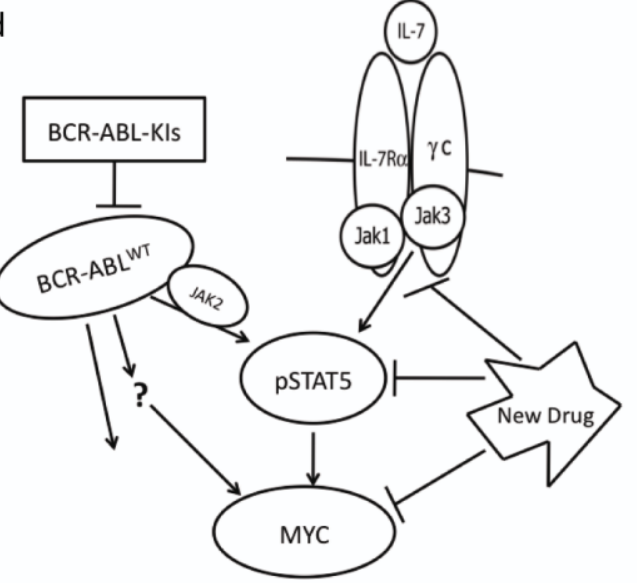

BCR-ABL- mutation independent dasatinib resistance model 
Over-expression of c-MYC resulted in a significant deterioration (100 fold) of dasatinib potency $\left(\mathrm{EC}_{50}\right)$ in a $\mathrm{BCR}-\mathrm{ABL}$ mutation-independent fashion (data shown later for convenience).

As an independent test of our hypothesis, we examined published biochemical profiles of agents for their binding affinity to kinase proteins [20], especially against BCR-ABL and JAK3 kinases, the latter being specific for mediating the effects of extracellular IL7 to intracellular compartment. Indeed, both dasatinib and nilotinib inhibit BCR-ABL at nanomolar concentrations, but were found to completely lack the ability to bind and inhibit JAK3 kinase.

\section{HT Drug Screens to Identify Agents with Activity against Ph+ ALL Cells Protected by Extracellular IL7 and T315I Mutation}

To search for novel agents with potent cellular activity against IL7-induced BCR-ABL-KI-resistant phenotypes, we screened 3200 well-annotated and mechanistically diverse bioactive agents, which included FDA-approved oncology and anti-infective drugs. In the primary screen, $\mathrm{Arf}^{/-} \mathrm{BCR}^{-\mathrm{ABL}}{ }^{\mathrm{WT}}$ LICs and $\mathrm{Arf}^{\prime-}$ BCR-ABL ${ }^{\mathrm{T} 315 \mathrm{I}}$ LICs were treated with a single concentration $(10 \mu \mathrm{M})$ of agents in the presence of IL7 $(0.85 \mathrm{ng} / \mathrm{mL})$. The quality of the screen was confirmed by receiver operating characteristic (ROC) analysis and other statistical metrics (Figures 4.8 to 4.12). The 627 compounds showing statistically significant activity ( $\geq 10 \%$ growth inhibition) were further augmented with available structural analogs and drugs of the same pharmacologic class to yield 706 active compounds. Concentration-response relationships were established for these compounds against Arf $^{/-}$BCR-ABL ${ }^{\text {WT }}$ LICs and drug-resistant $\mathrm{Arf}^{--}$BCR-ABL ${ }^{\mathrm{T} 315 \mathrm{I}}$ LICs in the presence of IL7, giving 229 validated hits with reasonable potency. All validated hits were equipotent in inhibiting the growth of Arf $^{\prime-}$ BCR-ABL ${ }^{\mathrm{WT}}$ LICs and Arf $^{/-}$BCR-ABL ${ }^{\mathrm{T} 315 \mathrm{I}}$ LICs. This set included 15 known cytotoxic antileukemic drugs (See supplemental data in Appendix), which enhance BCRABL-KI therapy in vivo [44-45, 63], thus validating the detection of appropriate phenotypic responses. Furthermore, cluster analysis (Figure 4.13) indicated that this set effectively sampled the space of all existing therapeutic classes (Figures 4.12 and 4.13). See supplemental data in Appendix for detailed annotation of all validated hits.

\section{A Common Anti-malarial Agent DHA Overcomes Multi-factorial BCR-ABL-KI Drug Resistance by Cell-extrinsic IL7 and Cell-intrinsic c-MYC Over-expression}

Given that the validated hits that overrode the IL7-protected leukemia phenotype contained compounds with varying mechanisms of action (Figure 4.13), and that increased c-MYC expression acts as a common node for primary BCR-ABL-KIresistance in 'high-risk' Ph+ ALL (Figures 4.6 and 4.7), we aimed to select those validated hits which may demonstrate selectivity to kill cancer cells with increased c-MYC expression and a known favorable toxicity profile in humans. 

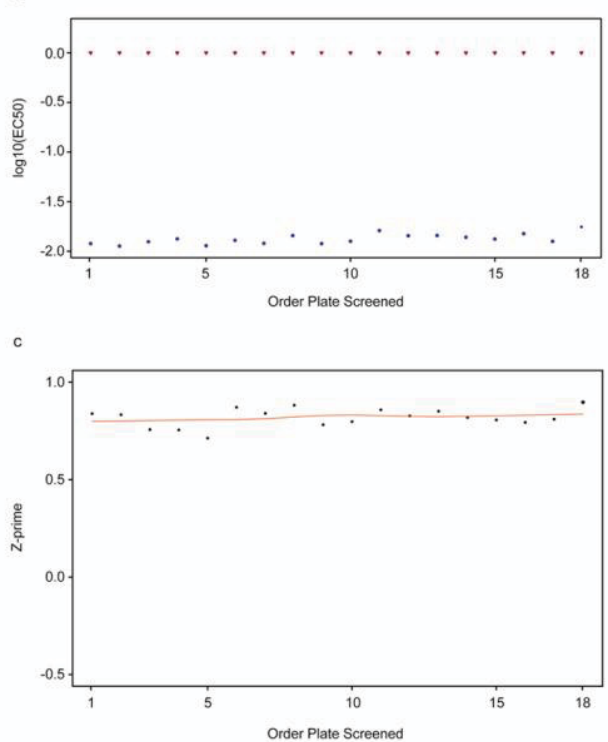
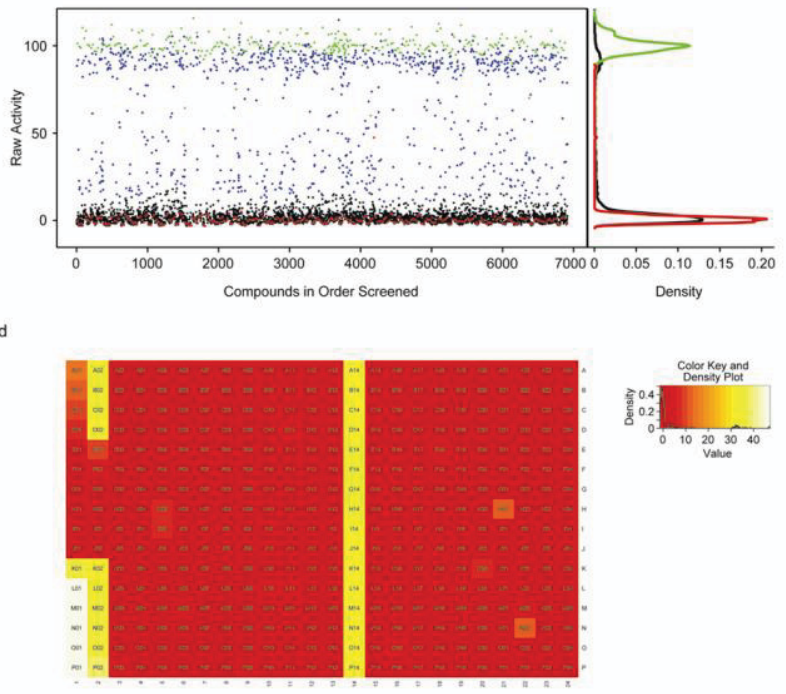

Figure 4.8. Quality control for the primary screen of 5600 agents against Arf $^{-1}$ BCR-ABL ${ }^{\text {WT }}$ LICs performed in the presence of $0.85 \mathrm{ng} / \mathrm{mL}$ IL7.

See Table 3.1 for well locations. (a) Distribution of $\mathrm{EC}_{50}$ of non-BCR-ABL-inhibiting reference compound staurosporine (calculated from wells 2A-2J in (d)). (b) Scatter plot of percent activity of the test compounds relative to controls. Good separation is seen between negative controls (red, DMSO-treated. max signal, column 13 in (d)) and positive controls (green, $300 \mathrm{nM}$ staurosporine, min signal, column 14 in (d)). Test compounds showing statistically significant activity relative to background noise (negative controls, red) are depicted in blue and inactive test compounds in black. Densities for positive, negative, and test compounds are shown on the right. (c) Distribution of Z' values for each plate in the primary screen. All Z' values throughout the run - average $=0.82$, lowest $=0.71$ and highest $=0.9$ (d) Heat map comparison of well activity averaged across all plates. Wells $1 \mathrm{~A}-1 \mathrm{~J}$ and $2 \mathrm{~A}-2 \mathrm{~J}$ show serial dilutions of dasatinib and staurosporine. In presence of IL7, average LIC growth inhibition of approx. $45 \%$ was achieved by $100 \mathrm{nM}$ dasatinib (well 1A), the later approaching maximum concentration achieved in human plasma. 

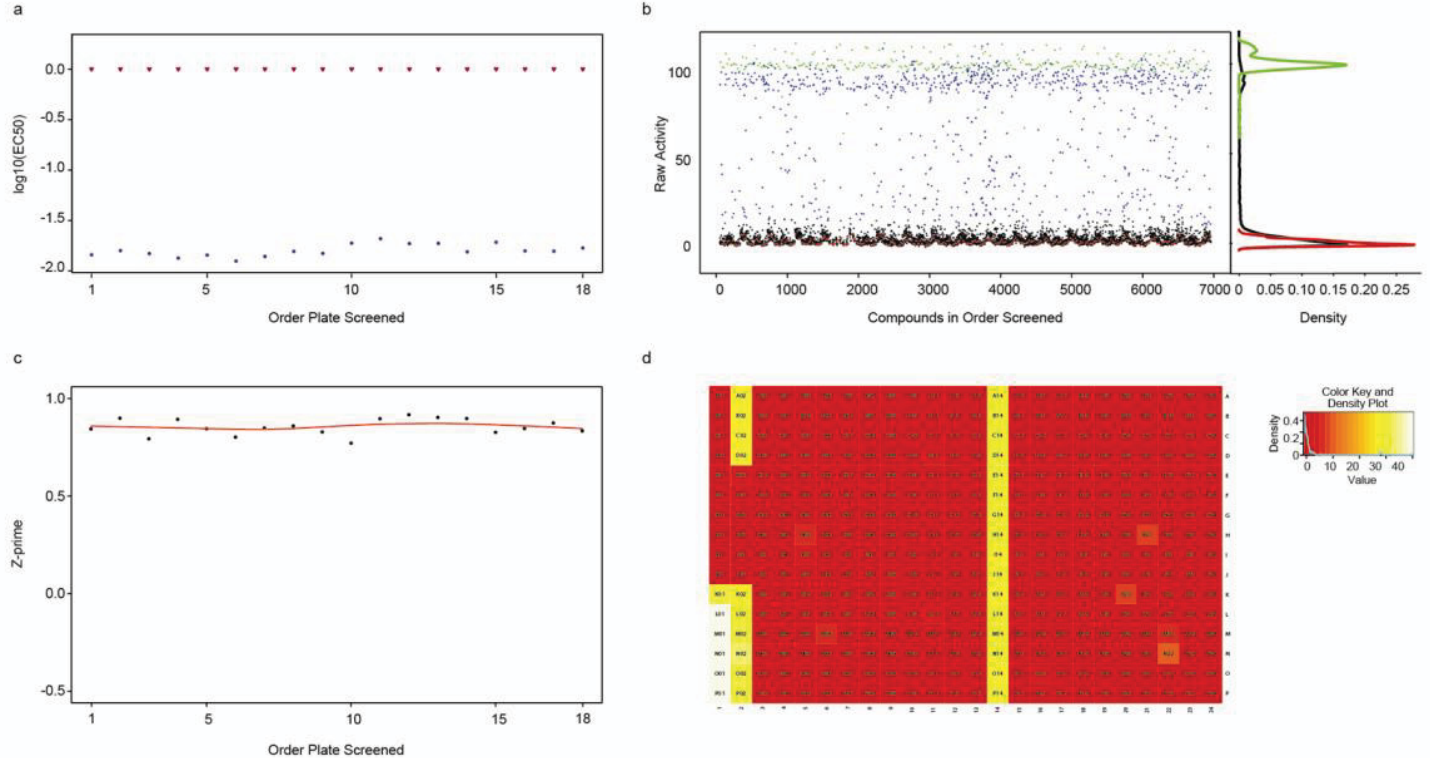

Figure 4.9. Quality control for primary screen of 5600 agents against $\mathrm{Arf}^{--} \mathrm{BCR}-$ $\mathrm{ABL}^{\mathrm{T} 315 \mathrm{~L}} \mathrm{LICs}$ performed in the presence of $0.85 \mathrm{ng} / \mathrm{mL}$ IL 7 .

(a) Distribution of $\mathrm{EC}_{50}$ of non-BCR-ABL-inhibiting reference compound staurosporine (calculated from wells 2A-2J in (d)). (b) Scatterplot of percent activity of the test compounds relative to controls. Good separation is seen between negative controls (red, DMSO-treated, max signal, column 13 in (d)) and positive controls (green, $300 \mathrm{nM}$ staurosporine, min signal, column 14 in (d)). Test compounds showing statistically significant activity relative to background noise (negative controls, red) are depicted in blue; inactive test compounds are black. (c) Distribution of Z' values for each plate in the primary screen. All Z' values throughout the run - average $=0.85$, lowest $=0.77$, and highest $=0.92$ (d) Heat map comparison of well activity averaged across all plates. Wells $1 \mathrm{~A}-1 \mathrm{~J}$ and $2 \mathrm{~A}-2 \mathrm{~J}$ show serial dilutions of dasatinib and staurosporine tested against BCR$\mathrm{ABL}^{\mathrm{T315I}}$ LICs. Although $100 \mathrm{nM}$ dasatinib (Well 1A) was totally ineffective, well 2A with highest staurosporine concentration $(300 \mathrm{nM})$ showed average LIC growth inhibition of approximately $100 \%$. 


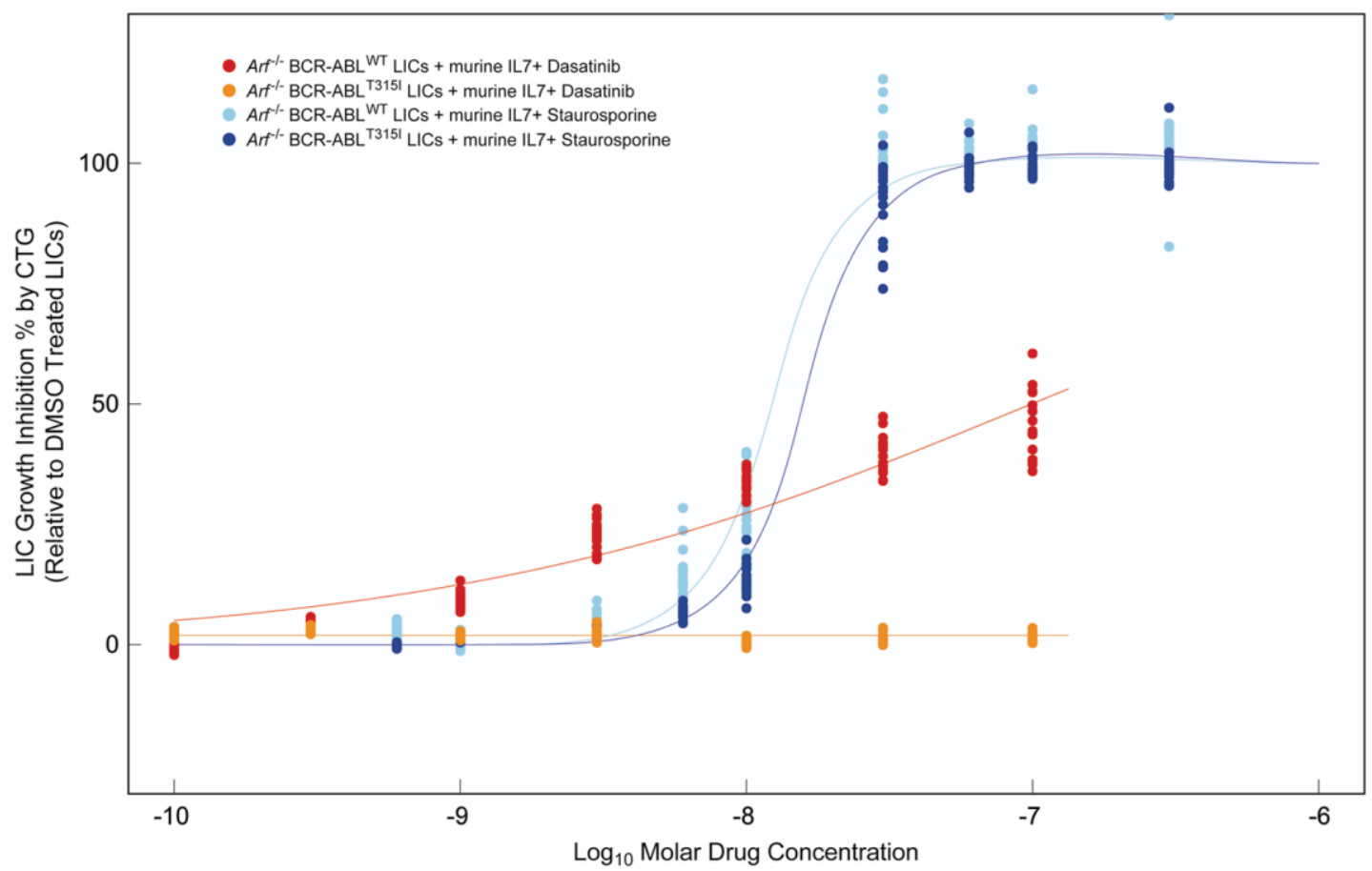

Figure 4.10. Primary high-throughput drug screening experiments captured BCR-ABL-KI drug-resistant phenotypes with high accuracy.

Distributions of independent dose response curves of dasatinib and staurosporine against $\mathrm{Arf}^{\prime-}$ BCR-ABL ${ }^{\mathrm{WT}}$ and $\mathrm{Arf}^{\prime-} \mathrm{BCR}^{-\mathrm{ABL}^{\mathrm{T}}{ }^{\mathrm{T} 15 \mathrm{I}} \text { LICs in the presence of } 0.85 \mathrm{ng} / \mathrm{mL} \text { IL7 }}$ calculated separately from all assay plates (wells $1 \mathrm{~A}-1 \mathrm{~J}$ and $2 \mathrm{~A}-2 \mathrm{~J}$ ) of primary drug screening experiments (also see Figures 4.8d and 4.9d) are presented. Both IL7-imparted dastinib-resistant phenotype in BCR-ABL ${ }^{\mathrm{WT}}$ LICs $\left(\mathrm{EC}_{50}\right.$ in nM: $95 \% \mathrm{CI}=86-116$, average $>100$ ) and BCR-ABL-mutation-imparted BCR-ABL-KI-resistant phenotype in BCR-ABL ${ }^{\mathrm{T} 315 \mathrm{I}} \mathrm{LICs}$, similar to Figure 4.2b, were consistently captured across all plates. Low interplate variability in calculated $\mathrm{EC}_{50}$ values was observed for the non-BCR-ABLtargeting reference compound staurosporine $\left(95 \% \mathrm{CI}\right.$ for $\mathrm{EC}_{50}$ in $\mathrm{nM}$ : WT $=11-12$, $\mathrm{T} 315 \mathrm{I}=15-16)$ and majority of the validated hits (refer the supplemental data in Appendix). 


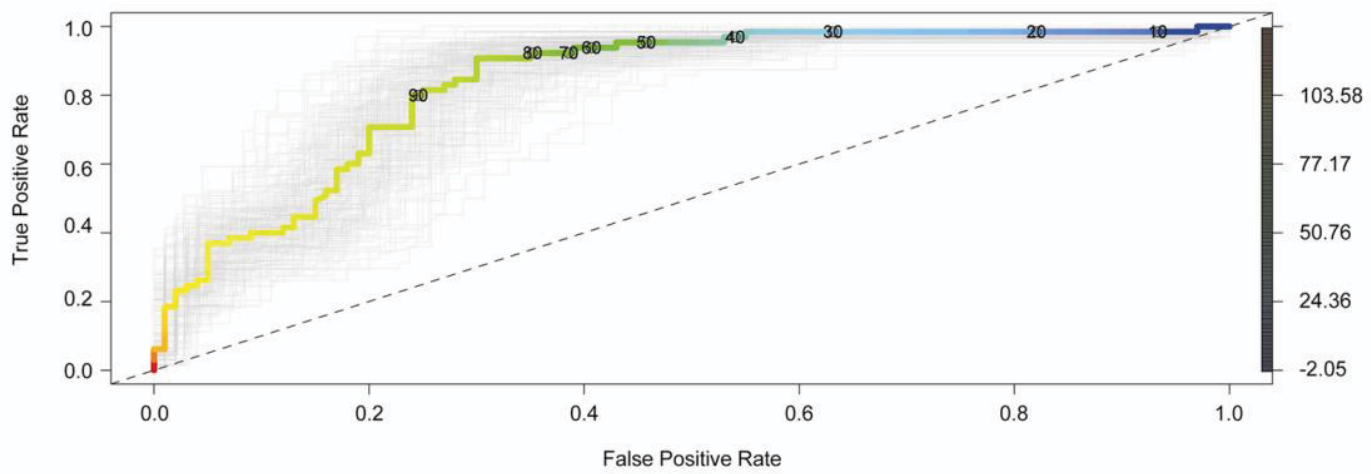

b T315I ROC AUC $=0.85(0.79-0.90)$

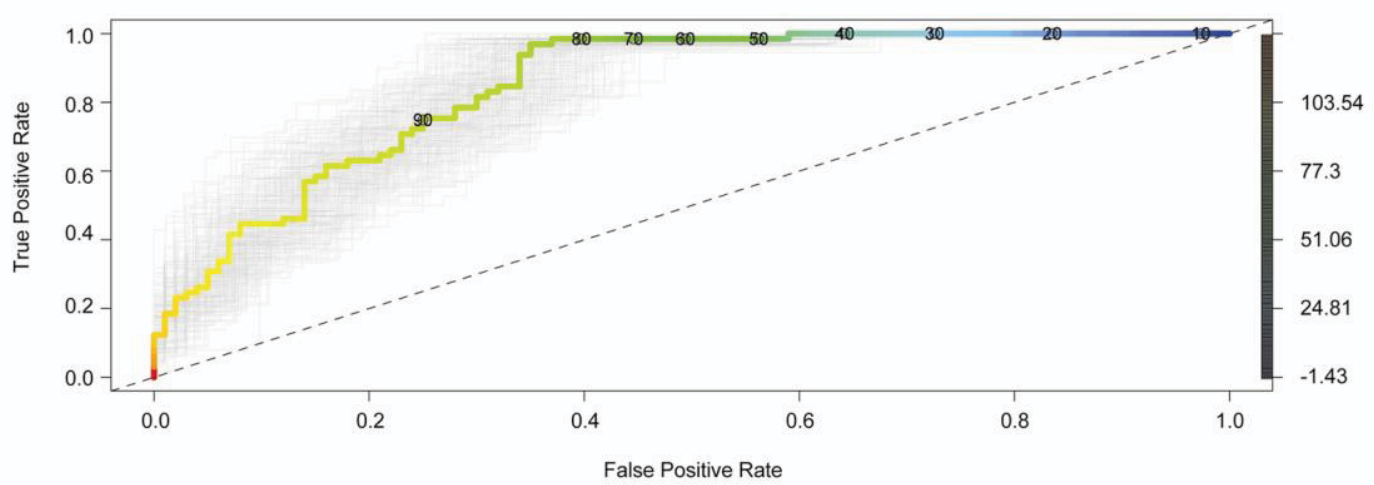

Figure 4.11. Receiver Operating Characteristic (ROC) analysis demonstrates that both (a) $\mathrm{Arf}^{-1}$ BCR-ABL ${ }^{\mathrm{WT}}$ and (b) $\mathrm{Arf}^{-1}$ BCR-ABL $^{\mathrm{T} 315 \mathrm{I}}$ LIC-based assays have high discriminatory power (AUC 0.83 and 0.85 , respectively).

The ROC curve is plotted as a function of \% activity, and is color coded according to the right $\mathrm{Y}$-axis. The ROC curves in gray are calculated from 200 bootstrap simulations. The dashed line corresponds to a random assay. 

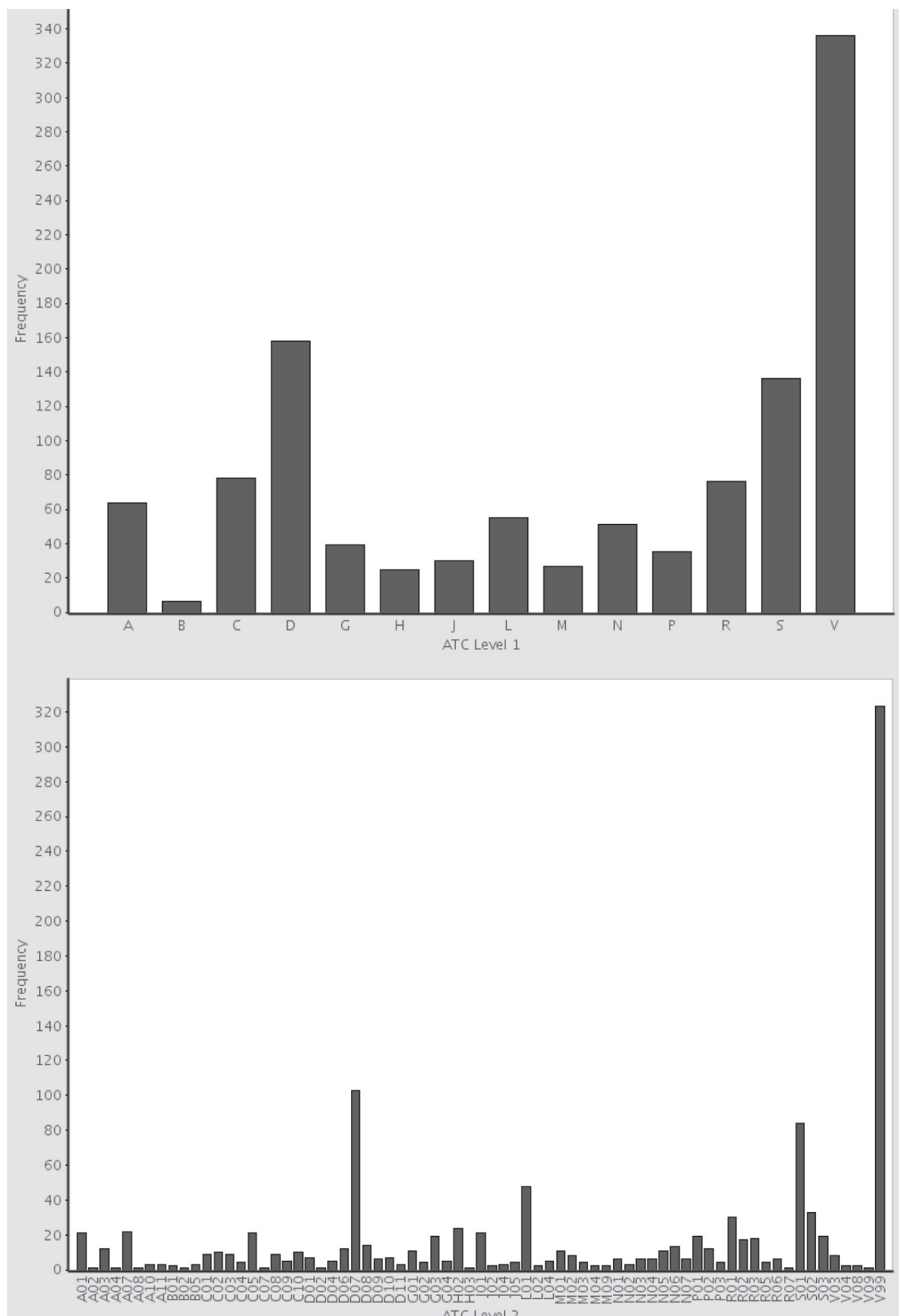

Figure 4.12. Frequency of ATC Level 1 (top) and ATC Level 2 (bottom) compounds for the 706 compounds submitted to secondary analysis. 


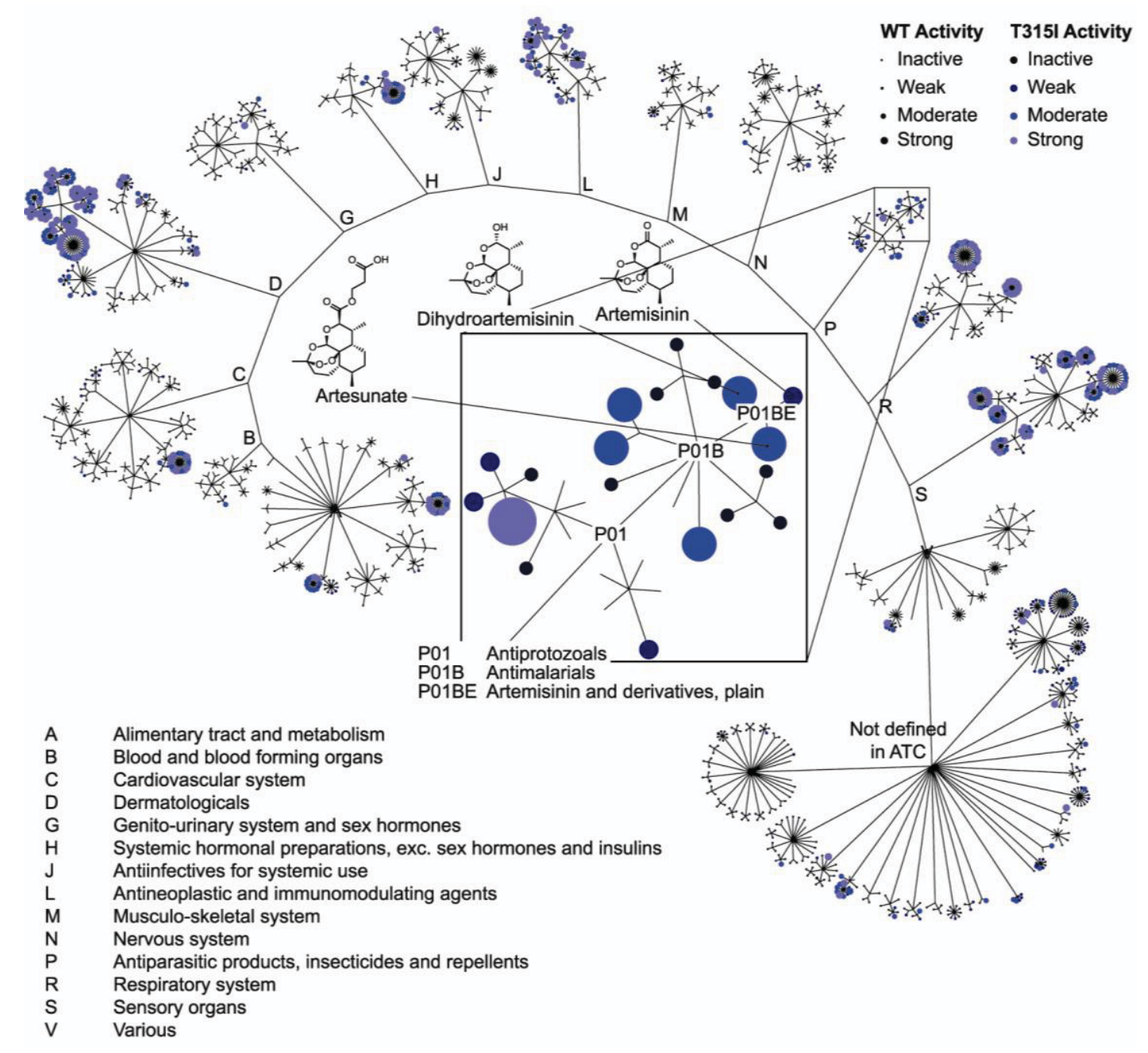

Figure 4.13. High-throughput drug screen to identify novel agents possessing activity against IL7-mediated early (primary) and BCR-ABL ${ }^{\text {MUTANT }}$-mediated late (secondary) BCR-ABL-KI drug-resistant phenotypes.

Clustering of validated hits using ATC therapeutic classification established by World Health Organization (WHO) (http://www.whocc.no/atc/structure and principles/). A single compound can be represented more than once because of multiple therapeutic indications, as is the case for the corticosteroids (highly active clusters in A, C, D, H, R, and $\mathrm{S}$ ). This demonstrates that the active compounds come from drugs used to treat many indications. For details on clustering methodology, please refer to Chapter 3, and for details on clustering analysis, please refer to supplemental data in Appendix and Figure 4.12. 
A correlative statistical analysis of NCI60 screening databases was performed. While a few validated hits demonstrated a significant positive $(+)$ correlation between their anti-cancer activity and c-MYC expression across 60 human cancer cell lines, the phenotypic sensitivity to dasatinib was found not to be correlated $(\mathrm{R}=-0.11$, where $\mathrm{R}$ is Pearsons coefficient of correlation) with c-MYC expression (Table 4.1). From the pool of validated hits demonstrating positive correlation with cellular c-MYC expression for their drug activity, we selected the artemisinin class of FDA-approved and very well-tolerated antimalarial drugs (ARTs) which included artemisinin, artesunate, and dihydroartemisinin (DHA) [104]. As a class, the ARTs were equipotent against $\mathrm{Arf}^{\prime-}$ BCR-ABL ${ }^{\mathrm{WT}}$ and $\mathrm{Arf}^{-/}$BCR-ABL ${ }^{\mathrm{T} 315 \mathrm{I}}$ LICs (Figure 4.14a) but the anti-LIC potency of artemisinin was much lower than that of artesunate or DHA. DHA and artesunate were also active against the human Ph+ ALL cell line SUP-B15 (Figure 4.14b) and had similar anti-Ph+ ALL potency in the presence of cell-extrinsic IL7 and cell-intrinsic over-expression of c-MYC, in stark contrast to dasatinib (Figure 4.14c). Studies have shown that DHA kills other genotypes of leukemic cells while selectively sparing normal human lymphocytes [105-106]. DHA, an active metabolite of many ARTs family members including artesunate, was chosen for further analysis because of its positive correlation for activity against tumor cells with relatively higher MYC expression $(\mathrm{R}=+0.5996, \mathrm{p}=0.0000433)$, in vitro potency, cellular selectivity, favorable pharmacokinetics, known bioavailability in hematopoietic system, and tolerability in all age groups.

\section{Dihydroartemisinin Possesses Single-agent In Vivo Activity against Ph+ ALL Phenotypes Demonstrating Primary and Acquired Dasatinib Resistance}

DHA monotherapy gave significant dose-dependent survival benefit in IL7-WT host-mice bearing low-burden $\mathrm{Arf}^{-1}$ BCR-ABL ${ }^{\mathrm{WT}}$ LICs (Figure 4.15a). Leukemic samples collected from the relapsing mice at the time of sacrifice revealed the complete absence of ABL KD mutations, which are often selected with dasatinib treatment in this model. DHA monotherapy also gave a similar response against low-burden $\mathrm{Arf}^{\prime-} \mathrm{BCR}$ $\mathrm{ABL}^{\mathrm{T} 315 \mathrm{I}}$ leukemia, a disease that is completely refractory to dasatinib (Figure 4.15b). Thus, DHA is active in vivo against murine $\mathrm{Ph}+\mathrm{ALL}$ through a mechanism independent of the BCR-ABL kinase.

\section{Cotreatment with Dihydroartemisinin Overcomes Host-mediated Primary Drug- resistance against Dasatinib and Eliminates Ph+ ALL In Vivo}

Given that $\mathrm{Arf}^{/-}$BCR-ABL ${ }^{\mathrm{WT}}$ leukemias in IL7-deficient mice were more sensitive to dasatinib (Figure 4.2e), and that DHA circumvented IL7 protection in vitro (Figure 4.14a and b) and displayed anti-Ph+ ALL activity in IL7-WT host mice (Figure 4.15a and b), the potential for synergy between the two drugs was evaluated. This evaluation was performed in cohorts of IL $7^{+/+}$immuno-competent mice bearing high burdens of Arf $^{/-}$BCR-ABL ${ }^{\text {WT }}$ Luc + LIC-driven leukemia (analogous to IL7 ${ }^{+/+}$[WT] 
Table 4.1. Priortization of validated hits according to known human tolerability and predicted involvement of c-MYC expression in their anti-cancer drug action.

\begin{tabular}{lll}
\hline $\begin{array}{l}\text { Hits Active against Dasatinib Resistant } \\
\text { Ph+ ALL Phenotypes* }\end{array}$ & $\begin{array}{l}\text { NSC No. for Hits } \\
\text { in DTP Database }\end{array}$ & $\begin{array}{l}\text { R (Coefficient of } \\
\text { Correlation)** }\end{array}$ \\
\hline GAMBOGIC ACID & 693702 & +0.59 \\
ARTESUNTE & 712571 & +0.59 \\
PATULIN & 8120 & +0.59 \\
DIHYDRO-ARTEMISINYL ETHER & 685989 & +0.57 \\
DIHYDRO-ARTEMISINYL ETHER & 685988 & +0.56 \\
ARTEMISININE & 369397 & +0.48 \\
MYCOPHENOLIC ACID & 129185 & +0.44 \\
CELASTROL & 70931 & +0.42 \\
THIRAM & 1771 & +0.40 \\
AKLAVINE HYDROCHLORIDE & 100290 & +0.40 \\
CHELERYTHRINE & 36405 & +0.36 \\
METHOTREXATE & 740 & +0.35 \\
EMETINE DIHYDROCHLORIDE & 33669 & +0.25 \\
ANTIMYCIN A & 186 & +0.06 \\
MITOXANTHRONE & 301739 & +0.04 \\
HYDROCHLORIDE & 26980 & +0.04 \\
MITOMYCIN C & 732517 & -0.11 \\
DASATINIB & &
\end{tabular}

* Examplary screening hits are presented for which preclinical and clinical toxicity data is available.

** $\mathrm{R}$ was calculated between drug activity and c-MYC expression across NCI60 human cancer cell lines, using NSC numbers as chemical identifiers for identified hits. Total sample size of $4461(4444+17)$ chemicals and drugs was used to calculate $p$ values; see Chapter 3 for details. 
Figure 4.14. Dihydroartemisinin of Artemisinin family of antimalarials circumvents dasatinib resistance induced by IL7, c-MYC overexpression and T315I mutation in vitro.

(a) $\mathrm{Arf}^{/-} \mathrm{BCR}-\mathrm{ABL}^{\mathrm{WT}}$ (triangles) and $\mathrm{Arf}^{\prime-}$ BCR-ABL ${ }^{\mathrm{T} 315 \mathrm{I}}$ (circles) LICs were treated with artemisinin, artesunate, dihydroartemisinin, or DMSO for $72 \mathrm{~h}$ in the absence (blue) or presence (red) of $1 \mathrm{ng} / \mathrm{mL}$ murine IL7, in triplicates. For each drug concentration, LIC growth (measured by the CTG-assay) was normalized to DMSO-treated LICs and fit using nonlinear regression (+/-sd). (b) Human Ph+ ALL SUP-B15 cells were treated with artesunate, dihydroartemisinin, or DMSO (no drug) for $72 \mathrm{~h}$ in the absence (blue) or presence (red) of $25 \mathrm{ng} / \mathrm{mL}$ human IL7. Viability was measured by the MTS assay.

Values were normalized to DMSO-treated cells. (c) Heads-up comparison of potencies of dihydroartemisin and dasatinib against (i) $\mathrm{Arf}^{-/} \mathrm{BCR}^{-\mathrm{ABL}}{ }^{\mathrm{WT}} \mathrm{LICs}+/-$ IL 7, (ii) $\mathrm{Arf}^{-/}$

BCR-ABL ${ }^{\text {WT }}$ LICs +/- c-MYC overexpression +/- IL7, and (iii) $A_{r f}{ }^{/-}$BCR-ABL $^{\text {WT }}$ LICs, using CTG-assay. 

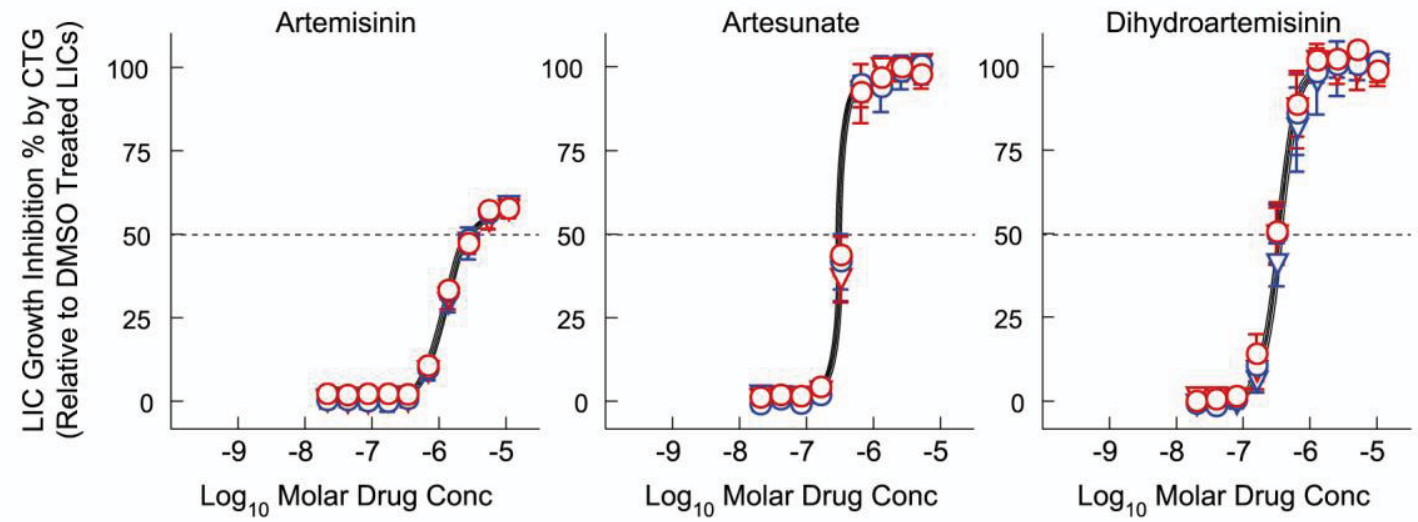

b
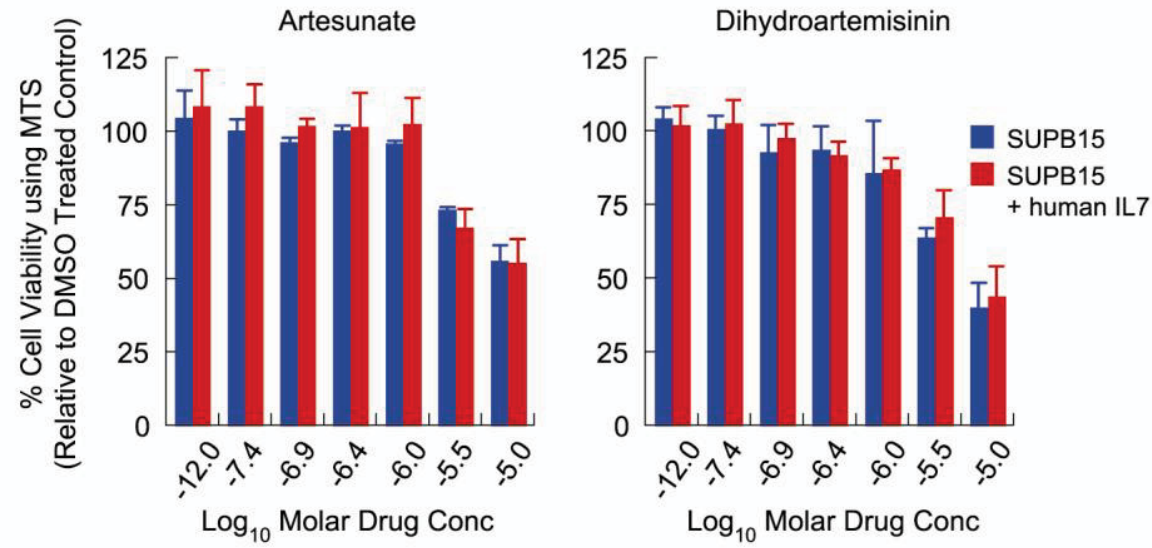

C

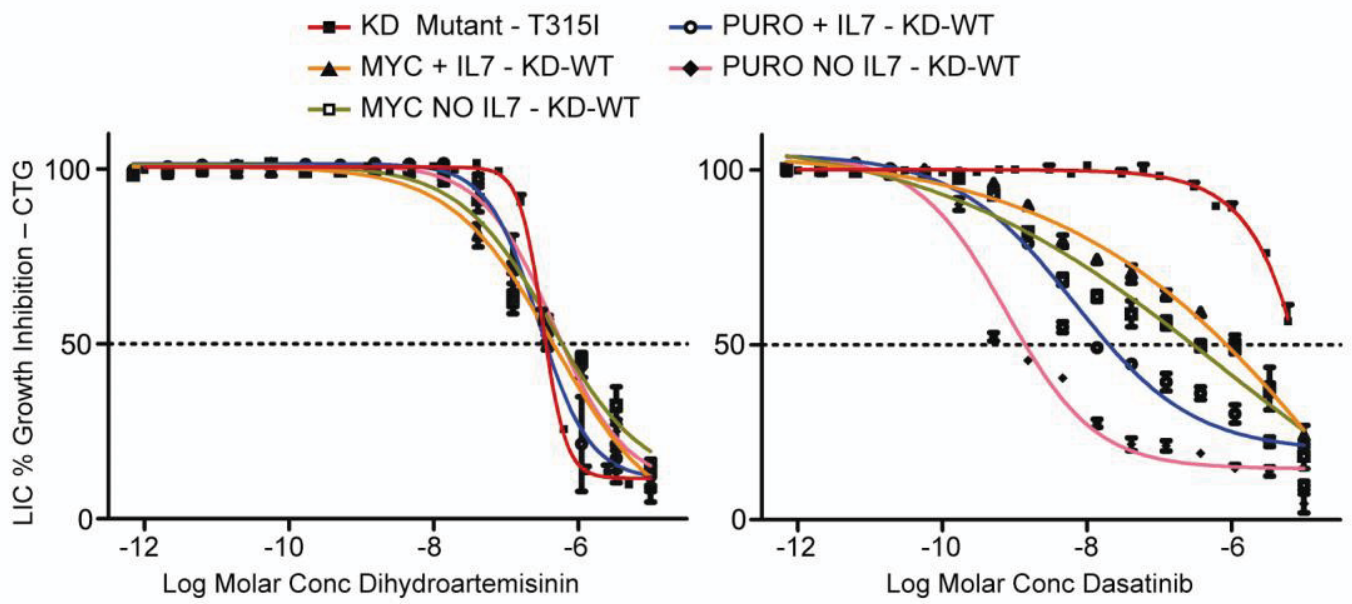




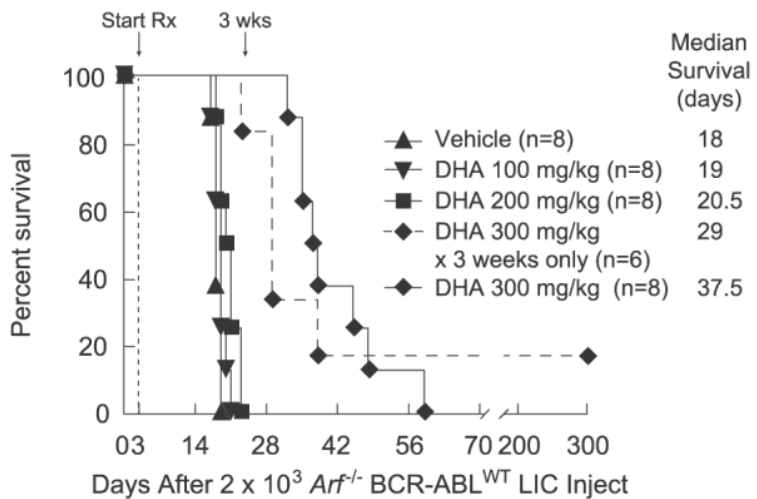

b

Treatment of Low Leukemia Burden

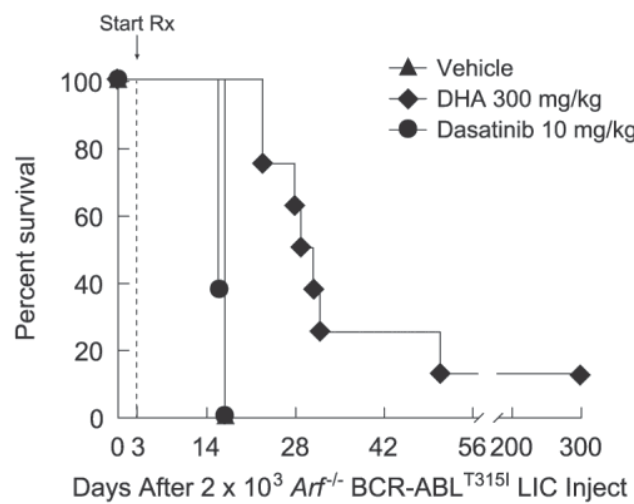

Figure 4.15. Dihydroartemisinin is active as a single agent against dasatinibresistant $\mathrm{Ph}+\mathrm{ALL}$ in $\mathrm{IL7} 7^{+/+}$host mice.

Kaplan-Meier survival curves showing the overall survival in response to therapy (survival benefit) in IL $7^{+/+}$B16 host mice that received day 0 tail vein injection of (a) $2 \times 10^{3} \mathrm{Arf}^{/-} \mathrm{BCR}-\mathrm{ABL}{ }^{\mathrm{WT}} \mathrm{Luc}+\mathrm{LICs}$; on day 3, once daily for 5 days of each week, vehicle, $100 \mathrm{mg} / \mathrm{kg}$ DHA, $200 \mathrm{mg} / \mathrm{kg}$ DHA, $300 \mathrm{mg} / \mathrm{kg}$ DHA, or $300 \mathrm{mg} / \mathrm{kg}$ DHA (for 3 weeks only) therapy was initiated in mice bearing low burdens of $\mathrm{BCR}-\mathrm{ABL}^{\mathrm{WT}}$-driven leukemia, or (b) $2 \times 10^{3} \mathrm{Arf}^{/-} \mathrm{BCR}-\mathrm{ABL}^{\mathrm{T} 315 \mathrm{I}} \mathrm{Luc}+\mathrm{LICs}$; on day 3, once daily for 5 days of each week, vehicle, $300 \mathrm{mg} / \mathrm{kg}$ DHA, or $10 \mathrm{mg} / \mathrm{kg}$ dasatinib was given to mice bearing low burdens of BCR-ABL ${ }^{\mathrm{T} 315 \mathrm{I}}$-driven leukemia. 
human patients carrying high burdens of Arf-deficient $\mathrm{p} 185^{\mathrm{BCR}-\mathrm{ABL}}$ pre-B cell leukemia at the time of diagnosis). Separate cohorts of animals were treated with dasatinib (10 $\mathrm{mg} / \mathrm{kg}$ ), DHA alone (200 or $300 \mathrm{mg} / \mathrm{kg}$ ) or binary combinations of dasatinib and DHA as shown in Figure 4.16a. Despite continuous monotherapy, dasatinib induced weak, transient primary responses. As a result, all animals harbored dasatinib-refractory persistent leukemia that inevitably led to relapse (Figure 4.16b) and caused death of all recipients within 6 weeks of initiating therapy (Figure 4.16a). DHA alone was completely ineffective $(200 \mathrm{mg} / \mathrm{kg})$ or weakly effective $(300 \mathrm{mg} / \mathrm{kg})$ in the high-burden leukemia model (Figure 4.16a). The combination of DHA and dasatinib significantly increased overall survival (Figure 4.16a), induced more rapid and deeper initial responses to dasatinib, and sensitized host-protected persistent leukemia to dasatinib (Figure 4.16b), in comparison to the treatment with either of the two drugs alone. Of the 12 mice given $200 \mathrm{mg} / \mathrm{kg}$ DHA and $10 \mathrm{mg} / \mathrm{kg}$ dasatinib, 3 survived long term, apparently free of all leukemia despite cessation of therapy. All mice $(n=12)$ given $300 \mathrm{mg} / \mathrm{kg}$ DHA and $10 \mathrm{mg} / \mathrm{kg}$ dasatinib survived the treatment period. After 4 weeks from the termination of combined therapy, 1 mouse succumbed to an isolated CNS relapse, which may be related to poor CNS penetration of dasatinib.[70-71]. Remarkably, the 14 surviving mice taken off the dasatinib and DHA combination regimens (3/12 at $200 \mathrm{mg} / \mathrm{kg}$ and 11/12 at $300 \mathrm{mg} / \mathrm{kg}$ DHA) for one year show no trace of leukemia. This data strongly suggested that drug combination cured the disease because in this leukemia model all recipient animals left untreated invariably succumb to fatal, full-blown leukemia within 4 weeks of injecting a very low numbers of LICs, as shown in Figure 4.1. Dasatinib-DHA regimens were well tolerated in leukemic and non-leukemic mice, in congruence with prior work utilizing similar dosages of DHA [93] and other ART family members [107-108] in rodents [93, 107] and sheep [108].

To extend our mechanistic understanding on DHA drug action, we identified genes whose expression levels were positively correlated with drug-sensitivity to DHA across 60 different human cancer cell lines, using public databases. We determined that the top 40 genes, which represent the drug-sensitivity signature for DHA in diverse human tumors, have a high and comparable expression in murine LICs and primary human $\mathrm{Ph}+\mathrm{ALL}$ leukemic samples with a positive correlation of $0.592(\mathrm{p}<0.001)$ as shown in Figure 4.17. This data predicts that DHA may maintain its anti-leukemic drug action against primary $\mathrm{Ph}+\mathrm{ALL}$ in human patients, similar to its efficacious results in our genetically relevant murine leukemia model (Figure 4.16a).

Next, to identify candidate genes targeted by DHA that plausibly resulted in overcoming host-cytokine mediated refractoriness against dasatinib, we compared gene signatures of DHA drug sensitivity (represented in Figure 4.17) and IL7-mediated dasatinib resistance (represented in Figure 4.6b). Three genes were found to overlap, namely MYC (probeset 1424942_a_at), Cabc1 (probeset 1417066 at, chaperone, ABC1 activity of bc1 complex like (S. pombe)) and Akap1 (probeset 1449019_at, A kinase (PRKA) anchor protein 1) using a 2-fold change as a cut-off in the IL7-resistance signature, whereas setting a 4-fold cut-off left MYC as the only overlapping gene between the two sigantures. This provided an alternative confirmation for our results. 
Figure 4.16. Dihydroartemisinin works synergistically with dasatinib to cure $\mathbf{P h}+$ ALL by improving primary responses to dasatinib.

(a) Kaplan-Meier survival curves showing the survival benefit in response to therapy in $\mathrm{IL}^{+/+}$B16 host mice that received day 0 tail vein injection of $2 \times 10^{5} \mathrm{Arf}^{/-} \mathrm{BCR}^{-\mathrm{ABL}}{ }^{\mathrm{WT}}$ Luc+ LICs. On day 7, six mouse groups bearing high burdens of BCR-ABL ${ }^{\mathrm{WT}}$-driven leukemia were started on daily treatment for 5 days of each week, using two-dose regimens -2 vehicles or 1 vehicle and 1 agent or 2 agents (see label), with 2 doses of each regimen given separately, $8 \mathrm{~h}$ apart, by oral gavage. On day 52, all therapy was terminated. (b) For 6 mouse groups in (a), separate graphs represent quantitative assessment of serial whole-animal luminescent signals $\left(\mathrm{p} / \mathrm{s} / \mathrm{cm}^{2} / \mathrm{sr}\right)$ acquired weekly. Solid lines represent imaging intensity of signal (y-axis) as a measure of leukemia burden from individual mice in the group, which is traced in real time on the x-axis. On day 7 , before initiating therapy, all 6 groups had comparable and high burden of leukemia. The horizontal dotted line represents the empirically determined lower limit of sensitivity for luminescent signal as an experimental cut-off for detectable levels of persistent leukemia. Rectangular gray box quantifies experimental primary therapeutic response to indicated regimens; box-width depicts approximate time (days) to induce maximal initial response and box-depth represents maximal decrease achieved in leukemia burden signal. Increasing depth and decreasing width indicate stronger and quicker primary responses, respectively. 

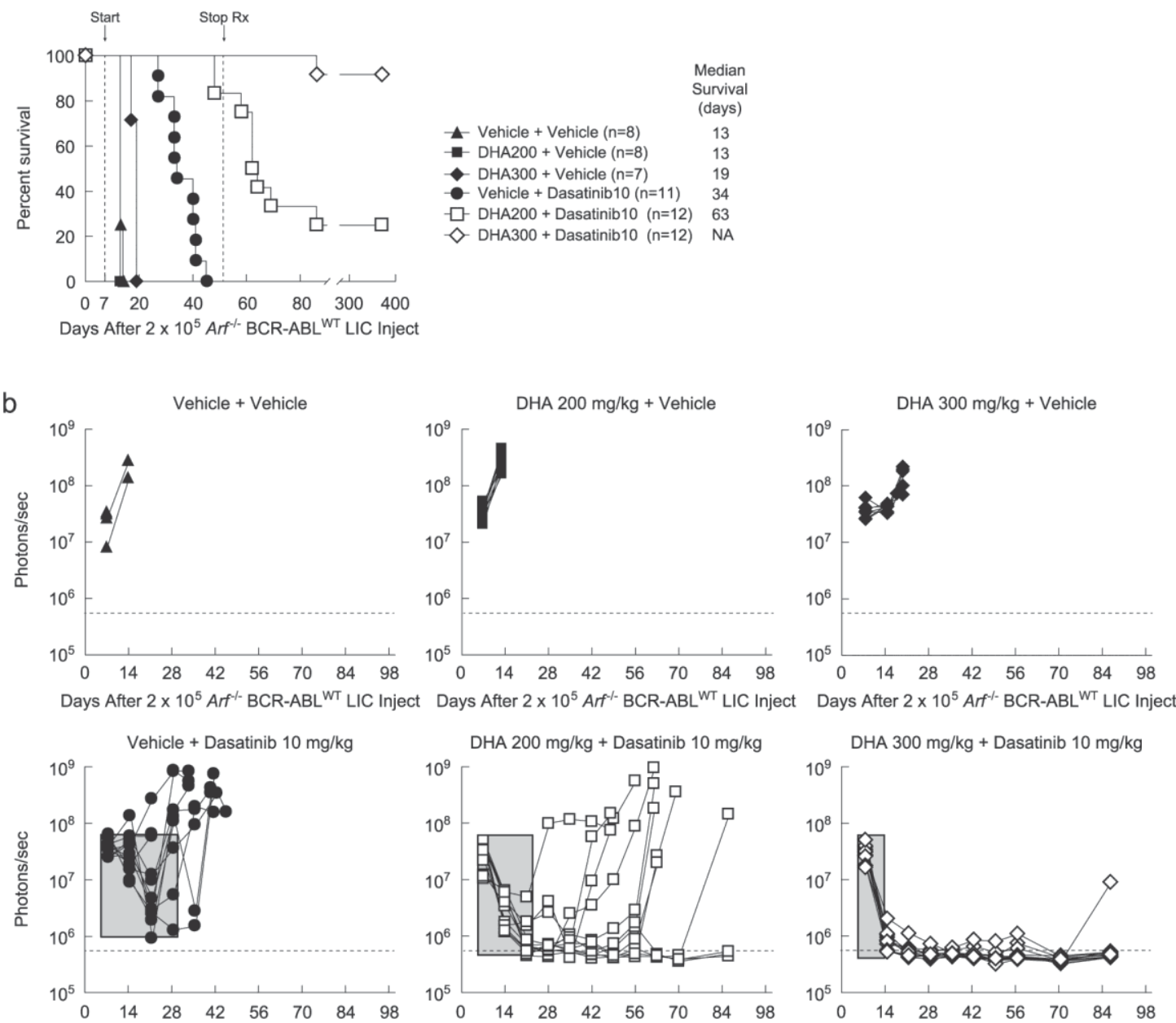

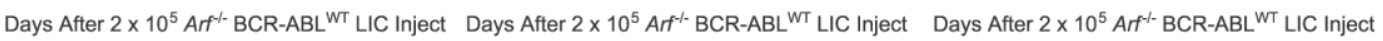


Figure 4.17. Statistical analyses to predict the activity of dihydroartemisin against human Ph+ ALL.

'Global anti-tumor activity signature' i.e. top 40 genes positively correlated with sensitivity to DHA across 60 human cancer cell lines, of dihydroartemisinin was statistically determined (y-axis) and then its normalized cellular expression ( $\mathrm{x}$-axis) was compared in LICs (mouse, black) and primary Ph+ ALL samples collected at the time of diagnosis (human, white). Calculated $\mathrm{R}=+0.592$ where $\mathrm{R}$ is the Pearson's coefficient of correlation and $\mathrm{p}<0.001$. 


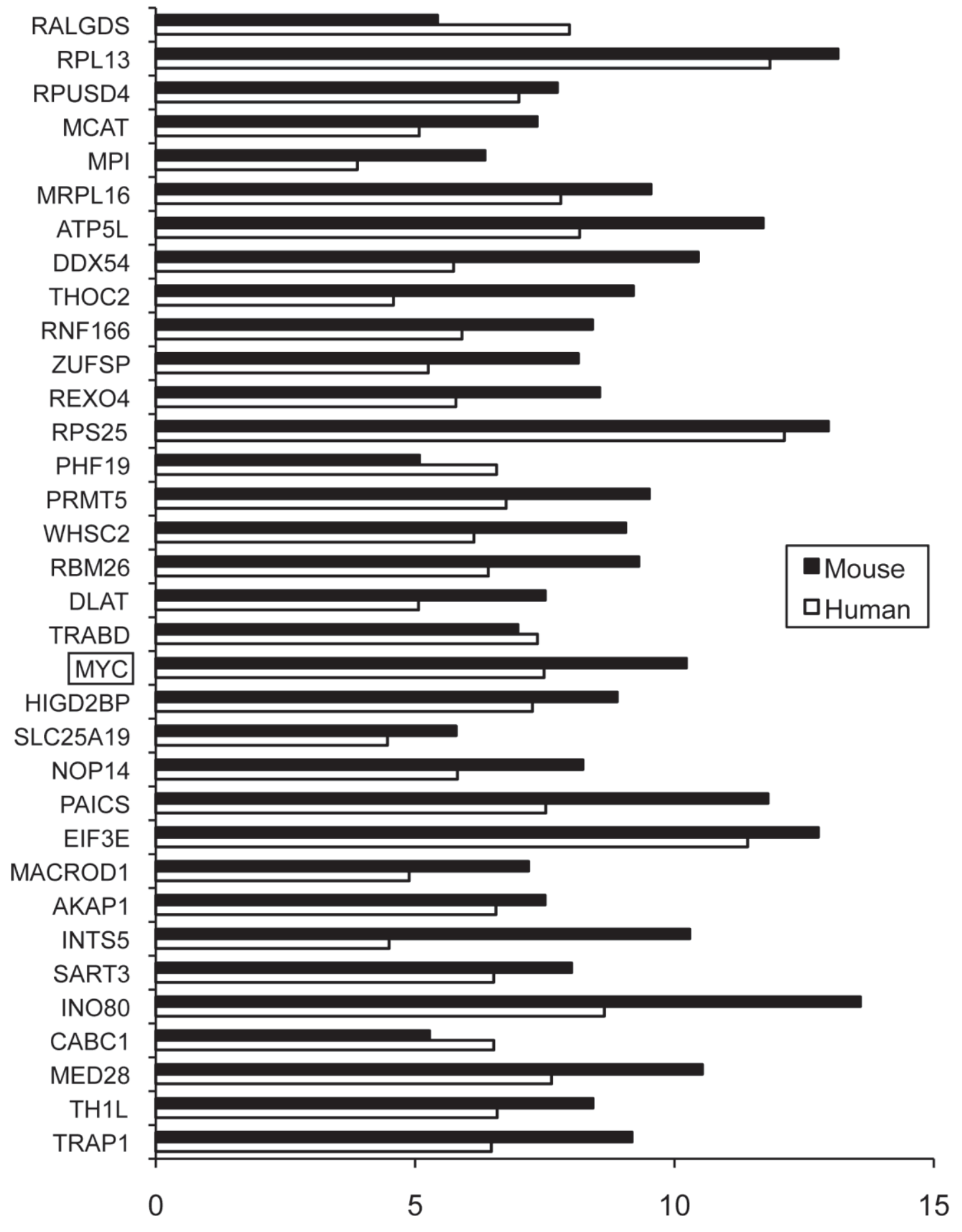




\section{CHAPTER 5. DISCUSSION AND CONCLUSIONS}

The findings of this study reinforce a key therapeutic paradigm that in vivo responsiveness to host-derived growth factors may shield autonomous cancer cells from chemotherapy-induced cell killing. Host-derived signaling likely contributes to the frustratingly poor clinical performances of some targeting agents with potent in vitro activities. We designed a multi-modality platform that interrogates host-tumor-drug interactions in an unbiased manner. In this forward-building HT method, a logical battery of cell-based screens performed across collections of host-factors, short hairpin (sh)-RNAs and chemical libraries accelerates the identification of host-factor(s) and associated cell-intrinsic molecular change(s) that can impart drug-resistant phenotype(s) and leverages it for the development of improved therapeutic strategies. Applying this HT method to Ph+ ALL, we identified i) IL7 as the dominant cytokine from the tumormicroenvironment that confers remarkable resistance to all FDA-approved BCR-ABLinhibitors, and ii) that anti-malarial drug DHA can reverse such host-IL7 mediated protection against dasatinib in vivo. This opens a novel potential avenue for clinical evaluation to treat human $\mathrm{Ph}+\mathrm{ALL}$ patients.

IL7 is essential for human T cell and post-fetal B cell development [109-110] and has a major contribution in the regulation and maintenance of mature T cells [111]. IL7 is constitutively produced by stromal cells in bone marrow, spleen, lymph nodes and thymus, among other tissues [112]. We propose a model in which IL7-rich niches harbor early persistent drug-refractory leukemias in humans [42, 44, 67, 113] and mice [63], with the presence of host-derived IL7 as the key driving factor behind the location of sequestration and the acquisition of resistance.

Results from cytokine-screens (Figure 4.2a) suggested that dasatinib resistance may be a more commonly prevalent host-dependent phenomenon, with IL7 [48, 114-115] as a predominant contributor in Ph+ ALL and other host-cytokines like TSLP [95], IL3 $[62,69,94]$, and IL4 playing a minor role. We show that although murine Arf $^{\prime-}$ BCR-ABL ${ }^{\text {WT }}$ LICs bearing, analogous to human $\mathrm{Ph}+\mathrm{ALL}$ at diagnosis, are irreversibly committed to cell death on treatment with dasatinib alone [56], cell-extrinsic IL7 reversibly sustains $\mathrm{Ph}+\mathrm{ALL}$, despite therapy that effectively inhibits BCR-ABL in vivo [55] by at least temporarily over-riding their dependence on BCR-ABL kinase for sustenance (Figure 4.4) [37, 67-68]. Our demonstration of poor dasatinib responsiveness against BCR-ABL ${ }^{\mathrm{WT}} \mathrm{Ph}+\mathrm{ALL}$ in $\mathrm{IL}^{+/+}$versus $\mathrm{IL}^{-/-}$host-mice (Figure 4.2e) supports a novel role for physiological IL7 in drug resistance in vivo. The primary protection by host-IL7, coupled with higher leukemia burden in Ph+ ALL compared to CML in the clinical settings, likely directly contributes to the greater BCR-ABL-KI refractoriness in $\mathrm{Ph}+\mathrm{ALL}$ relative to CML.

Leukemogenesis by BCR-ABL oncoprotein is a complex process, and depends on constitutive expression of STAT5 [59] and c-MYC [60-62] for initial transformation and sustenance of Ph+ ALL. BCR-ABL either directly or indirectly through JAK2 drives the phosphorylation of STAT5 (pSTAT5) in the cytoplasm. Activated STAT5 (pSTAT5) 
translocates to the nucleus and directly upregulates the expression of c-MYC [102]. This was confirmed by our failure to transform cellular bone marrow of STAT5-null mice with p185 isoform of BCR-ABL (data not shown). As demonstrated by others in human $\mathrm{Ph}+$ cells $[56,62,116]$, brief BCR-ABL inhibition in BCR-ABL ${ }^{\text {WT }}$ LICs by dasatinib (no IL7) significantly decreased the levels of both pSTAT5 protein and expression of MYC and MYC target genes, committing leukemia blasts to death. The specificity of IL7 to attenuate the potency of only BCR-ABL inhibitors in both murine and human $\mathrm{Ph}+\mathrm{ALL}$ cells but not of those agents which inhibit targets other than BCR-ABL suggested that IL7 likely rescues a particular signaling pathway downstream of BCR-ABL to impart primary drug resistance. Further mechanistic studies revealed a model in which dasatinib blocks the essential BCR-ABL-JAK2-pSTAT5-c-MYC signaling but presence of cell-extrinsic IL7 rescues the critical expression of c-MYC and its target genes by phosphorylating STAT5 through parallel IL7-JAK3-pSTAT5-cMYC signaling, in a BCR-ABL-independent fashion [102]. The primary IL7-protection against dasatinib is relatively lower than the molecular drug resistance conferred by T315I mutation (Figure 4.2b). Dasatinib does not inhibit BCR-ABL kinase or decreease the cellular level of pSTAT5 or c-MYC in BCR-ABL ${ }^{\mathrm{T} 315 \mathrm{I}}$ human cells [117]. Interestingly, STAT activity [117-120] and expression of c-MYC [121] has recently been used as an explanation for resistance to imatinib. c-MYC has also been implicated by others in stroma-mediated drug resistance [66, 122]. Reinforcing our primary drug resistance model (Figure 4.7d), we show that overexpression of c-MYC imparts $>100$ fold resistance against dasatinib in the absence of both leukemia-extrinsic IL7 and leukemia-intrinsic ABL KD mutations. We propose c-MYC as a cellular node for primary drug resistance against BCR-ABL targeted therapy in $\mathrm{Ph}+\mathrm{ALL}$.

A recent mechanistic study demonstrated direct de-repression of c-MYC by IKAROS haploinsufficiency in pre-B cells [103]. Our preliminary experiments depict that modeling the functional loss of IKAROS in BCR-ABL ${ }^{\mathrm{WT}}$ pre-B LICs by expression of DN IKAROS mutant, which increases c-MYC expression, imparts cell-intrinsic refractoriness against dasatinib and imatinib. IKAROS haploinsufficiency is a poor human prognostic marker [123] and is detected in leukemia samples obtained (at the time of diagnosis) from a large percentage of $\mathrm{Ph}+\mathrm{ALL}$ patients $[75,123]$ but almost never in chronic phase CML patients $[75,124]$. Thus, IKAROS haploinsufficiency may also play a role in relative primary BCR-ABL-KI-resistance in $\mathrm{Ph}+\mathrm{ALL}$, similar and in addition to that by host-IL7, which is a non-redundant cytokine present in all human patients.

In summary, repeated BCR-ABL-KI exposures in host-IL7 rich niches result in 'darwinian' enrichment of leukemic blasts with relatively higher levels of pSTAT5 and c-MYC, and/or genetic mutations in IKAROS and Arf loci, accelerating the progression of disease. Selective multi-clonal clonal expansions follow $[72,125]$, leading to variance in therapeutic responses among human patients, similarly, BCR-ABL-mutations also accumulate with continuous therapy [50-51]. Acquisition of these complex changes impart niche-independent cell-intrinsic drug-resistance and clinically manifest as frank leukemic relapses $[72,125]$. In lieu of our findings, high pSTAT5 protein and c-MYC expression level and loss of IKAROS in $\mathrm{Ph}+$ lymphoblasts, and elevated levels of host IL7 can potentially be used as surrogates of primary BCR-ABL-KI drug resistance, 
pending further preclinical and clinical confirmation. These markers may facilitate personalized medicine in future.

In keeping with critical homeostatic role of IL7 in immune-reconstitution [111], IL7 levels dramatically vary, inverse to individual patient's absolute lymphocyte counts [126-127]. Current management of Ph+ ALL patients with BCR-ABL-KI-combined protocols deploy maximum tolerated dose (MTD)-guided conventional chemotherapy agents, high-dose corticosteroids, allo-HSCT and immune-suppressants, all of which induce profound iatrogenic-lymphopenias [126-127], and activate the immune IL7-axis [126-127] which can decrease the efficacy of BCR-ABL targeted therapy. Dasatinib therapy induces robust survival-benefit in human $\mathrm{Ph}+\mathrm{ALL}$ patients with prolonged lymphocytosis, which may arise from their inherent low levels of IL7, and vice versa [128]. Also, human Ph+ ALL patients with increased levels of pSTAT5, whose phosphorylation is triggered by IL7, respond poorly to imatinib therapy [129]. Collectively, these data strongly support the proposition that host-IL7 protection phenomenon contributes to BCR-ABL-KI-resistance in human patients. This suggests strategic amendments to current leukemia management to avoid prolonged lymphopenias.

From therapeutic perspective, BCR-ABL focused drug discovery aims at achieving more potent BCR-ABL inhibition, and views the escaped kinase activity due to ABL KD mutations, especially T315I, as an integral part of the problem [17-18]. In contrast, our host-tumor-drug interaction studies underscore the necessity for 'complementing' pharmacological BCR-ABL-inhibition with strategies/drugs that circumvent primary host-cytokine protection and its associated cellular changes (pSTAT5 levels and c-MYC expression) to accomplish long-lasting responses in $\mathrm{Ph}+\mathrm{ALL}$. A clinically-validated strategy to overcome drug resistance is to concomitantly use multiple agents whose mechanism of action, and therefore of drug resistance, vary significantly, as illustrated by the success of the combination chemotherapy regimens against various cancers and the highly active anti-retroviral therapy (HAART) against HIV [130]. Likewise, we adapted drug resistant phenotypes, mediated by host-IL7 and BCR-ABL T315I mutation, into our flexible cell-based platform and deployed a HT screen across a library of 3200 known drugs and natural products to select active agents, ideal for combined use with BCR-ABL-KIs in vivo.

Our approach accurately confirmed activities of various chemotherapeutic agents and majority of clinically used anti-leukemic drugs, many of which enhance the clinical and preclinical performances of BCR-ABL-KIs [44-45, 63]. In addition, several novel chemotypes were identified to be active against primary host-IL7 and late BCR-ABL mutation mediated drug-resistant phenotypes of $\mathrm{Ph}+\mathrm{ALL}$. These chemically-diverse hits are non-BCR-ABL specific and inhibit cellular targets 'downstream' of BCR-ABL or outside its ATP-binding or allosteric site. By definition, such agents cannot be identified by biochemical kinase binding assays or target-drug structural modeling, which form the backbone of target-focused approach. Our results predict that an ideal BCR-ABL-KI should additionally inhibit JAK1/JAK3 kinase activity to prevent relay of drug-refractory signal from the activated IL7 surface receptor to the inside of leukemic lymphoblast. A review of public kinase-binding profiles of imatinib and dasatinib [20] confirms their 
inability to inhibit JAK1/JAK3 kinase activity. Until BCR-ABL-KIs with additional JAK3-inhibiting activity become available, empirically determined drug combinations of $\mathrm{BCR}-\mathrm{ABL}-\mathrm{KIs}$ are bound to play an important bridging role in $\mathrm{Ph}+\mathrm{ALL}$ therapeutics.

In support of our phenotypic complementation strategy, we characterized the anti-leukemia activities of one screening hit, the orally-bioavailable, well-tolerated antimalarial drug DHA [104], in detail. The biggest challenges in curing Ph+ ALL using BCR-ABL-KIs are poor primary responses, persistence of disease, invariant relapses, and late BCR-ABL mutational resistance. DHA, acting mechanistically independent of BCR-ABL-KIs (Figures 4.14 and 4.15), synergistically corrects the deficiencies associated with dasatinib monotherapy (Figure 4.16b), and achieves near-complete long-term survival in a robust murine model of human Ph+ ALL. Distinct from its anti-malarial drug action [131-132], drug sensitivity to the ARTs family across various cancer types appears to correlate with relative c-MYC expression. While it has been shown that DHA induces apoptosis in cancer cells by selectively accelerating the degradation of c-MYC [133], our preliminary bio-physical studies (Singh and Kriwacki, unpublished), using previously validated assays, indicate that DHA directly binds to basic-helix-loop-helix domain of c-MYC. Most importantly, as described earlier (Figures 4.6 and 4.7), c-MYC is the converging node of primary dasatinib resistance, and targeting $\mathrm{c}-\mathrm{MYC}$, using dominant negative approaches, has been validated as a BCR-ABL-independent therapeutic intervention in pre-B Ph+ ALL [60-61]. Thus, DHA might augment BCR-ABL-KI therapy by accelerating the degradation of c-MYC, thereby countering the protective effects of IL 7.

The accelerated characterization of anti-malarial drug DHA for a new anti-leukemic use was linked to our original decision of testing only a small but focused collection of very well-characterized bioactive agents and drugs, and their mechanistic evaluation in DTP screening drug databases [89, 134-135]. In past, this strategy has made immense and rapid contributions in the field of mechanistic drug discovery [90-91, 134, 136-137]. In addition to ARTs family, anti-tumor activity of gambogic acid and mycophenolic acid was also found to be statistically correlated with high MYC expression in NCI60 human cancer cell lines (Table 4.1), and could be considered to target dasatinib-resistant Ph+ ALL. However, direct involvement of c-MYC in the mechanism of action of these two alternative agents remains to be experimentally proven [138-140]. Further, Gambogic acid, a natural resin product, has never been tested in humans, and mycophenolic acid is an FDA-approved agent but induces immunosuppression and lymphopenia $[138,141]$. In contrast, DHA selectively kills leukemic cells while sparing normal lymphocytes [105-106], therefore is less likely to induce host-IL7. We determined that gene signature predicting sensitivity to DHA across human tumors of 9 different tissue lineages (Figure 4.17) is expressed with a high degree of similarity in murine LICs and primary samples obtained from human $\mathrm{Ph}+\mathrm{ALL}$ patients. It is noteworthy that DHA-dasatinib combination is significantly more efficacious than our previous experience with triple combination between dasatinib, dexamethasone and asparaginase [63]. Collectively, these data strongly support the testing of efficacy of BCR-ABL-KI/DHA co-therapy for the clinical management of human patients with high-risk $\mathrm{Ph}+\mathrm{ALL}$. 
Complementing BCR-ABL and alternative target-focused drug pipelines [15-16, 19-20, 23] with our host-tumor-drug screening approach can timely predict mechanisms and biomarkers of clinical drug-resistance. A holistic approach encompassing these features can improve the prioritization of hits and facilitate improvement in design of agents against $\mathrm{Ph}+\mathrm{ALL}$ and other cancers in future [15-16, 19-20, 23]. Broader implementation of reported screening model will also address two important and inter-related issues which remain pending. First, whether the overly-specific targeting nature of the modern era oncology drugs, relative to the non-specific drugs, renders them more vulnerable to compensatory (cell extrinsic/intrinsic) parallel pathways in the tumor cells, and second, what is the extent of this problem among the current clinically approved agents. 


\section{LIST OF REFERENCES}

1. Weinstein, I.B., Cancer. Addiction to oncogenes--the Achilles heal of cancer. Science, 2002. 297(5578): p. 63-4.

2. Harris, T.J. and F. McCormick, The molecular pathology of cancer. Nat Rev Clin Oncol, 2010. 7(5): p. 251-65.

3. Weinstein, I.B. and A.K. Joe, Mechanisms of disease: Oncogene addiction--a rationale for molecular targeting in cancer therapy. Nat Clin Pract Oncol, 2006. 3(8): p. 448-57.

4. Ciardiello, F. and G. Tortora, EGFR antagonists in cancer treatment. N Engl J Med, 2008. 358(11): p. 1160-74.

5. $\quad \mathrm{Du}, \mathrm{J} .$, et al., Bead-based profiling of tyrosine kinase phosphorylation identifies SRC as a potential target for glioblastoma therapy. Nat Biotechnol, 2009. 27(1): p. $77-83$.

6. Karp, J.E. and J.E. Lancet, Tipifarnib in the treatment of newly diagnosed acute myelogenous leukemia. Biologics, 2008. 2(3): p. 491-500.

7. Motzer, R.J., et al., Sunitinib versus interferon alfa in metastatic renal-cell carcinoma. N Engl J Med, 2007. 356(2): p. 115-24.

8. Chalandon, Y. and J. Schwaller, Targeting mutated protein tyrosine kinases and their signaling pathways in hematologic malignancies. Haematologica, 2005. 90(7): p. 949-68.

9. Guo, J., et al., Phase II, open-label, single-arm trial of imatinib mesylate in patients with metastatic melanoma harboring c-Kit mutation or amplification. $\mathrm{J}$ Clin Oncol, 2011. 29(21): p. 2904-9.

10. Pao, W. and J. Chmielecki, Rational, biologically based treatment of EGFRmutant non-small-cell lung cancer. Nat Rev Cancer, 2010. 10(11): p. 760-74.

11. Ghoreschi, K., A. Laurence, and J.J. O'Shea, Selectivity and therapeutic inhibition of kinases: to be or not to be? Nat Immunol, 2009. 10(4): p. 356-60.

12. Soria, J.C., et al., Phase I safety, pharmacokinetic and pharmacodynamic trial of BMS-599626 (AC480), an oral pan-HER receptor tyrosine kinase inhibitor, in patients with advanced solid tumors. Ann Oncol, 2011.

13. Krause, D.S. and R.A. Van Etten, Tyrosine kinases as targets for cancer therapy. N Engl J Med, 2005. 353(2): p. 172-87. 
14. Reichardt, P. and M. Montemurro, Clinical experience to date with nilotinib in gastrointestinal stromal tumors. Semin Oncol, 2011. 38 Suppl 1: p. S20-7.

15. Metz, J.T., et al., Navigating the kinome. Nat Chem Biol, 2011. 7(4): p. 200-2.

16. Zhang, X. and A. Fernandez, In silico drug profiling of the human kinome based on a molecular marker for cross reactivity. Mol Pharm, 2008. 5(5): p. 728-38.

17. O'Hare, T., et al., AMN107: tightening the grip of imatinib. Cancer Cell, 2005. 7(2): p. 117-9.

18. Zhang, J., et al., Targeting Bcr-Abl by combining allosteric with ATP-binding-site inhibitors. Nature, 2010. 463(7280): p. 501-6.

19. Liu, Q., et al., Kinome-wide selectivity profiling of ATP-competitive mTOR (mammalian target of rapamycin) inhibitors and characterization of their binding kinetics. J Biol Chem, 2012.

20. Fabian, M.A., et al., A small molecule-kinase interaction map for clinical kinase inhibitors. Nat Biotechnol, 2005. 23(3): p. 329-36.

21. Yap, T.A., C.P. Carden, and S.B. Kaye, Beyond chemotherapy: targeted therapies in ovarian cancer. Nat Rev Cancer, 2009. 9(3): p. 167-81.

22. Rosenzweig, S.A., Acquired resistance to drugs targeting receptor tyrosine kinases. Biochem Pharmacol, 2011.

23. Dannenberg, J.H. and A. Berns, Drugging drug resistance. Cell, 2010. 141(1): p. $18-20$.

24. Weeraratna, A.T., RAF around the edges--the paradox of BRAF inhibitors. $\mathrm{N}$ Engl J Med, 2012. 366(3): p. 271-3.

25. Wong, S. and O.N. Witte, The BCR-ABL story: bench to bedside and back. Annu Rev Immunol, 2004. 22: p. 247-306.

26. Kurzrock, R., et al., Philadelphia chromosome-positive leukemias: from basic mechanisms to molecular therapeutics. Ann Intern Med, 2003. 138(10): p. 81930.

27. Reya, T., et al., Stem cells, cancer, and cancer stem cells. Nature, 2001. 414(6859): p. 105-11.

28. O'Brien, S.G., et al., Imatinib compared with interferon and low-dose cytarabine for newly diagnosed chronic-phase chronic myeloid leukemia. N Engl J Med, 2003. 348(11): p. 994-1004. 
29. Druker, B.J., et al., Five-year follow-up of patients receiving imatinib for chronic myeloid leukemia. N Engl J Med, 2006. 355(23): p. 2408-17.

30. Bjorkholm, M., et al., Success story of targeted therapy in chronic myeloid leukemia: a population-based study of patients diagnosed in Sweden from 1973 to 2008. J Clin Oncol, 2011. 29(18): p. 2514-20.

31. Hughes, T.P., et al., Frequency of major molecular responses to imatinib or interferon alfa plus cytarabine in newly diagnosed chronic myeloid leukemia. $\mathrm{N}$ Engl J Med, 2003. 349(15): p. 1423-32.

32. de Lavallade, H., et al., Imatinib for newly diagnosed patients with chronic myeloid leukemia: incidence of sustained responses in an intention-to-treat analysis. J Clin Oncol, 2008. 26(20): p. 3358-63.

33. Wolff, N.C., et al., The CNS is a sanctuary for leukemic cells in mice receiving imatinib mesylate for Bcr/Abl-induced leukemia. Blood, 2003. 101(12): p. 5010-3.

34. Martinelli, G., et al., Prediction of response to imatinib by prospective quantitation of $B C R-A B L$ transcript in late chronic phase chronic myeloid leukemia patients. Ann Oncol, 2006. 17(3): p. 495-502.

35. Savona, M. and M. Talpaz, Getting to the stem of chronic myeloid leukaemia. Nat Rev Cancer, 2008. 8(5): p. 341-50.

36. Lane, S.W., D.T. Scadden, and D.G. Gilliland, The leukemic stem cell niche: current concepts and therapeutic opportunities. Blood, 2009. 114(6): p. 1150-7.

37. Hamilton, A., et al., Chronic myeloid leukemia stem cells are not dependent on Bcr-Abl kinase activity for their survival. Blood, 2012. 119(6): p. 1501-10.

38. Gratwohl, A., et al., Allogeneic hematopoietic stem cell transplantation for chronic myeloid leukemia in Europe 2006: transplant activity, long-term data and current results. An analysis by the Chronic Leukemia Working Party of the European Group for Blood and Marrow Transplantation (EBMT). Haematologica, 2006. 91(4): p. 513-21.

39. Passweg, J.R., et al., Validation and extension of the EBMT Risk Score for patients with chronic myeloid leukaemia (CML) receiving allogeneic haematopoietic stem cell transplants. Br J Haematol, 2004. 125(5): p. 613-20.

40. Jain, N. and K. van Besien, Chronic myelogenous leukemia: role of stem cell transplant in the imatinib era. Hematol Oncol Clin North Am, 2011. 25(5): p. 1025-48, vi.

41. Velev, N., et al., Stem cell transplantation for patients with chronic myeloid leukemia resistant to tyrosine kinase inhibitors with BCR-ABL kinase domain mutation T315I. Cancer, 2010. 116(15): p. 3631-7. 
42. Talpaz, M., et al., Dasatinib in imatinib-resistant Philadelphia chromosomepositive leukemias. N Engl J Med, 2006. 354(24): p. 2531-41.

43. Williams, R.T., M.F. Roussel, and C.J. Sherr, Arf gene loss enhances oncogenicity and limits imatinib response in mouse models of Bcr-Abl-induced acute lymphoblastic leukemia. Proc Natl Acad Sci U S A, 2006. 103(17): p. 668893.

44. Ravandi, F., et al., First report of phase 2 study of dasatinib with hyper-CVAD for the frontline treatment of patients with Philadelphia chromosome-positive ( $\mathrm{Ph}+)$ acute lymphoblastic leukemia. Blood, 2010. 116(12): p. 2070-7.

45. de Labarthe, A., et al., Imatinib combined with induction or consolidation chemotherapy in patients with de novo Philadelphia chromosome-positive acute lymphoblastic leukemia: results of the GRAAPH-2003 study. Blood, 2007. 109(4): p. 1408-13.

46. Ribera, J.M., et al., Concurrent intensive chemotherapy and imatinib before and after stem cell transplantation in newly diagnosed Philadelphia chromosomepositive acute lymphoblastic leukemia. Final results of the CSTIBES02 trial. Haematologica, 2009. 95(1): p. 87-95.

47. Zaliova, M., et al., Quantification of fusion transcript reveals a subgroup with distinct biological properties and predicts relapse in BCR/ABL-positive ALL: implications for residual disease monitoring. Leukemia, 2009. 23(5): p. 944-51.

48. Williams, R.T., W. den Besten, and C.J. Sherr, Cytokine-dependent imatinib resistance in mouse BCR-ABL+, Arf-null lymphoblastic leukemia. Genes Dev, 2007. 21(18): p. 2283-7.

49. Wei, Y., et al., Not all imatinib resistance in $C M L$ are BCR-ABL kinase domain mutations. Ann Hematol, 2006. 85(12): p. 841-7.

50. Jones, D., et al., Kinase domain point mutations in Philadelphia chromosomepositive acute lymphoblastic leukemia emerge after therapy with BCR-ABL kinase inhibitors. Cancer, 2008. 113(5): p. 985-94.

51. Nicolini, F.E., et al., Epidemiologic study on survival of chronic myeloid leukemia and Ph(+) acute lymphoblastic leukemia patients with BCR-ABL T315I mutation. Blood, 2009. 114(26): p. 5271-8.

52. Khalil, S.H., et al., Molecular monitoring of response to imatinib (Glivec) in chronic myeloid leukemia patients: experience at a tertiary care hospital in Saudi Arabia. Genet Test Mol Biomarkers, 2010. 14(1): p. 67-74.

53. Cortes, J., et al., Dynamics of BCR-ABL kinase domain mutations in chronic myeloid leukemia after sequential treatment with multiple tyrosine kinase inhibitors. Blood, 2007. 110(12): p. 4005-11. 
54. Wongboonma, W., W. Thongnoppakhun, and C.U. Auewarakul, BCR-ABL kinase domain mutations in tyrosine kinase inhibitors-naive and-exposed Southeast Asian chronic myeloid leukemia patients. Exp Mol Pathol, 2012.

55. Luo, F.R., et al., Dasatinib (BMS-354825) pharmacokinetics and pharmacodynamic biomarkers in animal models predict optimal clinical exposure. Clin Cancer Res, 2006. 12(23): p. 7180-6.

56. Snead, J.L., et al., Acute dasatinib exposure commits Bcr-Abl-dependent cells to apoptosis. Blood, 2009. 114(16): p. 3459-63.

57. Williams, R.T. and C.J. Sherr, The INK4-ARF (CDKN2A/B) locus in hematopoiesis and BCR-ABL-induced leukemias. Cold Spring Harb Symp Quant Biol, 2008. 73: p. 461-7.

58. Williams, R.T. and C.J. Sherr, BCR-ABL and CDKN2A: a dropped connection. Nat Rev Cancer, 2008. 8(7): p. 563; author reply 563.

59. Hoelbl, A., et al., Stat5 is indispensable for the maintenance of bcr/abl-positive leukaemia. EMBO Mol Med, 2010. 2(3): p. 98-110.

60. Sawyers, C.L., W. Callahan, and O.N. Witte, Dominant negative MYC blocks transformation by ABL oncogenes. Cell, 1992. 70(6): p. 901-10.

61. Sawyers, C.L., The role of myc in transformation by BCR-ABL. Leuk Lymphoma, 1993. 11 Suppl 1: p. 45-6.

62. Xie, S., et al., Jak2 is involved in c-Myc induction by Bcr-Abl. Oncogene, 2002. 21(47): p. 7137-46.

63. Boulos, N., et al., Chemotherapeutic agents circumvent emergence of dasatinibresistant BCR-ABL kinase mutations in a precise mouse model of Philadelphia chromosome-positive acute lymphoblastic leukemia. Blood, 2011. 117(13): p. 3585-95.

64. Quintas-Cardama, A., H. Kantarjian, and J. Cortes, Flying under the radar: the new wave of BCR-ABL inhibitors. Nat Rev Drug Discov, 2007. 6(10): p. 834-48.

65. Hughes, T., et al., Impact of baseline BCR-ABL mutations on response to nilotinib in patients with chronic myeloid leukemia in chronic phase. J Clin Oncol, 2009. 27(25): p. 4204-10.

66. McMillin, D.W., et al., Tumor cell-specific bioluminescence platform to identify stroma-induced changes to anticancer drug activity. Nat Med, 2010. 16(4): p. 483-9. 
67. Weisberg, E., et al., Stromal-mediated protection of tyrosine kinase inhibitortreated BCR-ABL-expressing leukemia cells. Mol Cancer Ther, 2008. 7(5): $\mathrm{p}$. $1121-9$.

68. Hiwase, D.K., et al., Blocking cytokine signaling along with intense Bcr-Abl kinase inhibition induces apoptosis in primary CML progenitors. Leukemia, 2010. 24(4): p. 771-8.

69. Kim, H.P., A.E. Frankel, and D.E. Hogge, A diphtheria toxin interleukin-3 fusion protein synergizes with tyrosine kinase inhibitors in killing leukemic progenitors from BCR/ABL positive acute leukemia. Leuk Res, 2010. 34(8): p. 1035-42.

70. Lagas, J.S., et al., Brain accumulation of dasatinib is restricted by P-glycoprotein $(A B C B 1)$ and breast cancer resistance protein $(A B C G 2)$ and can be enhanced by elacridar treatment. Clin Cancer Res, 2009. 15(7): p. 2344-51.

71. Chen, Y., et al., P-glycoprotein and breast cancer resistance protein influence brain distribution of dasatinib. J Pharmacol Exp Ther, 2009. 330(3): p. 956-63.

72. Notta, F., et al., Evolution of human BCR-ABL1 lymphoblastic leukaemiainitiating cells. Nature, 2011. 469(7330): p. 362-7.

73. Mullighan, C.G., et al., Genome-wide analysis of genetic alterations in acute lymphoblastic leukaemia. Nature, 2007. 446(7137): p. 758-64.

74. Mullighan, C.G., et al., Failure of CDKN2A/B (INK4A/B-ARF)-mediated tumor suppression and resistance to targeted therapy in acute lymphoblastic leukemia induced by BCR-ABL. Genes Dev, 2008. 22(11): p. 1411-5.

75. Mullighan, C.G., et al., BCR-ABL1 lymphoblastic leukaemia is characterized by the deletion of Ikaros. Nature, 2008. 453(7191): p. 110-4.

76. Liu, P., et al., Expression of dominant-negative Ikaros isoforms and associated genetic alterations in Chinese adult patients with leukemia. Ann Hematol, 2012.

77. Onishi, M., et al., Identification and characterization of a constitutively active STAT5 mutant that promotes cell proliferation. Mol Cell Biol, 1998. 18(7): p. $3871-9$.

78. McKeller, R.N., et al., The Arf tumor suppressor gene promotes hyaloid vascular regression during mouse eye development. Proc Natl Acad Sci U S A, 2002. 99(6): p. 3848-53.

79. Shah, N.P., et al., Multiple BCR-ABL kinase domain mutations confer polyclonal resistance to the tyrosine kinase inhibitor imatinib (ST1571) in chronic phase and blast crisis chronic myeloid leukemia. Cancer Cell, 2002. 2(2): p. 117-25. 
80. Kocisko, D.A., et al., New inhibitors of scrapie-associated prion protein formation in a library of 2000 drugs and natural products. J Virol, 2003. 77(19): p. 10288-94.

81. Weissmann, C. and A. Aguzzi, Approaches to therapy of prion diseases. Annu Rev Med, 2005. 56: p. 321-44.

82. Team, R.D.C., R: A language and environment for statistical computing. 2009, R Foundation for Statistical Computing: Vienna, Austria.

83. Ritz, C. and J.C. Streibig, Bioassay Analysis using R. J Statist Software, 2005. 12 (5)(5): p. 22.

84. Sing, T., et al., ROCR: visualizing classifier performance in R. Bioinformatics, 2005. 21(20): p. 3940-1.

85. Smoot, M.E., et al., Cytoscape 2.8: new features for data integration and network visualization. Bioinformatics, 2011. 27(3): p. 431-2.

86. Ekins, S., et al., A combined approach to drug metabolism and toxicity assessment. Drug Metab Dispos, 2006. 34(3): p. 495-503.

87. Meng, L.H., et al., Activation of aminoflavone (NSC 686288) by a sulfotransferase is required for the antiproliferative effect of the drug and for induction of histone gamma-H2AX. Cancer Res, 2006. 66(19): p. 9656-64.

88. Rao, V.A., et al., Batracylin (NSC 320846), a dual inhibitor of DNA topoisomerases I and II induces histone gamma-H2AX as a biomarker of DNA damage. Cancer Res, 2007. 67(20): p. 9971-9.

89. Zaharevitz, D.W., et al., COMPARE: a web accessible tool for investigating mechanisms of cell growth inhibition. J Mol Graph Model, 2002. 20(4): p. $297-$ 303.

90. Paull, K.D., et al., Display and analysis of patterns of differential activity of drugs against human tumor cell lines: development of mean graph and COMPARE algorithm. J Natl Cancer Inst, 1989. 81(14): p. 1088-92.

91. Paull, K.D., et al., Identification of novel antimitotic agents acting at the tubulin level by computer-assisted evaluation of differential cytotoxicity data. Cancer Res, 1992. 52(14): p. 3892-900.

92. $\mathrm{Hu}, \mathrm{Y} .$, et al., Targeting multiple kinase pathways in leukemic progenitors and stem cells is essential for improved treatment of Ph+ leukemia in mice. Proc Natl Acad Sci U S A, 2006. 103(45): p. 16870-5.

93. Nontprasert, A., et al., Assessment of the neurotoxicity of oral dihydroartemisinin in mice. Trans R Soc Trop Med Hyg, 2002. 96(1): p. 99-101. 
94. Dorsey, J.F., et al., Interleukin-3 protects Bcr-Abl-transformed hematopoietic progenitor cells from apoptosis induced by Bcr-Abl tyrosine kinase inhibitors. Leukemia, 2002. 16(9): p. 1589-95.

95. Brown, V.I., et al., Thymic stromal-derived lymphopoietin induces proliferation of pre-B leukemia and antagonizes $m$ TOR inhibitors, suggesting a role for interleukin-7Ralpha signaling. Cancer Res, 2007. 67(20): p. 9963-70.

96. Jalkanen, S.E., et al., Poor cytokine-induced phosphorylation in chronic myeloid leukemia patients at diagnosis is effectively reversed by tyrosine kinase inhibitor therapy. Exp Hematol, 2011.39(1): p. 102-113 e1.

97. Carlesso, N., D.A. Frank, and J.D. Griffin, Tyrosyl phosphorylation and DNA binding activity of signal transducers and activators of transcription (STAT) proteins in hematopoietic cell lines transformed by Bcr/Abl. J Exp Med, 1996. 183(3): p. 811-20.

98. Shuai, K., et al., Constitutive activation of STAT5 by the BCR-ABL oncogene in chronic myelogenous leukemia. Oncogene, 1996. 13(2): p. 247-54.

99. Hantschel, O., et al., BCR-ABL uncouples canonical JAK2-STAT5 signaling in chronic myeloid leukemia. Nat Chem Biol, 2012. 8(3): p. 285-93.

100. Tanaka, R., et al., Activity of the multitargeted kinase inhibitor, AT9283, in imatinib-resistant BCR-ABL-positive leukemic cells. Blood, 2010. 116(12): p. 2089-95.

101. van der Plas, D.C., et al., Interleukin-7 signaling in human B cell precursor acute lymphoblastic leukemia cells and murine BAF3 cells involves activation of STAT1 and STAT5 mediated via the interleukin-7 receptor alpha chain. Leukemia, 1996. 10(8): p. 1317-25.

102. Lord, J.D., et al., The IL-2 receptor promotes lymphocyte proliferation and induction of the $c-m y c, b c l-2$, and bcl-x genes through the trans-activation domain of Stat5. J Immunol, 2000. 164(5): p. 2533-41.

103. Ma, S., et al., Ikaros and Aiolos inhibit pre-B-cell proliferation by directly suppressing c-Myc expression. Mol Cell Biol, 2010. 30(17): p. 4149-58.

104. White, N.J., Qinghaosu (artemisinin): the price of success. Science, 2008. 320(5874): p. 330-4.

105. Lai, H. and N.P. Singh, Selective cancer cell cytotoxicity from exposure to dihydroartemisinin and holotransferrin. Cancer Lett, 1995. 91(1): p. 41-6.

106. Singh, N.P. and H.C. Lai, Synergistic cytotoxicity of artemisinin and sodium butyrate on human cancer cells. Anticancer Res, 2005. 25(6B): p. 4325-31. 
107. Duthaler, U., T.A. Smith, and J. Keiser, In vivo and in vitro sensitivity of Fasciola hepatica to triclabendazole combined with artesunate, artemether, or OZ78. Antimicrob Agents Chemother, 2010. 54(11): p. 4596-604.

108. Duthaler, U., et al., Evaluation of the pharmacokinetic profile of artesunate, artemether and their metabolites in sheep naturally infected with Fasciola hepatica. Vet Parasitol, 2011.

109. von Freeden-Jeffry, U., et al., Lymphopenia in interleukin (IL)-7 gene-deleted mice identifies IL-7 as a nonredundant cytokine. J Exp Med, 1995. 181(4): p. 1519-26.

110. Parrish, Y.K., et al., IL-7 Dependence in human B lymphopoiesis increases during progression of ontogeny from cord blood to bone marrow. J Immunol, 2009. 182(7): p. 4255-66.

111. Schluns, K.S., et al., Interleukin-7 mediates the homeostasis of naive and memory CD8 T cells in vivo. Nat Immunol, 2000. 1(5): p. 426-32.

112. Repass, J.F., et al., IL7-hCD25 and IL7-Cre BAC transgenic mouse lines: new tools for analysis of IL-7 expressing cells. Genesis, 2009. 47(4): p. 281-7.

113. Lee, S., et al., The extent of minimal residual disease reduction after the first 4week imatinib therapy determines outcome of allogeneic stem cell transplantation in adults with Philadelphia chromosome-positive acute lymphoblastic leukemia. Cancer, 2009. 115(3): p. 561-70.

114. Sasson, S.C., et al., IL-7 receptor is expressed on adult pre-B-cell acute lymphoblastic leukemia and other B-cell derived neoplasms and correlates with expression of proliferation and survival markers. Cytokine, 2010. 50(1): p. 58-68.

115. Williams, R.T. and C.J. Sherr, The ARF tumor suppressor in acute leukemias: insights from mouse models of Bcr-Abl-induced acute lymphoblastic leukemia. Adv Exp Med Biol, 2007. 604: p. 107-14.

116. Nunoda, K., et al., Identification and functional signature of genes regulated by structurally different ABL kinase inhibitors. Oncogene, 2007. 26(28): p. 4179-88.

117. Nelson, E.A., et al., The STAT5 inhibitor pimozide decreases survival of chronic myelogenous leukemia cells resistant to kinase inhibitors. Blood, 2011. 117(12): p. 3421-9.

118. Page, B.D., et al., Small Molecule STAT5-SH2 Domain Inhibitors Exhibit Potent Antileukemia Activity. J Med Chem, 2012.

119. Jilani, I., et al., Phosphorylation levels of BCR-ABL, CrkL, AKT and STAT5 in imatinib-resistant chronic myeloid leukemia cells implicate alternative pathway usage as a survival strategy. Leuk Res, 2008. 32(4): p. 643-9. 
120. Hantschel, O., et al., BCR-ABL uncouples canonical JAK2-STAT5 signaling in chronic myeloid leukemia. Nat Chem Biol, 2012.

121. Albajar, M., et al., MYC in Chronic Myeloid Leukemia: Induction of Aberrant DNA Synthesis and Association with Poor Response to Imatinib. Mol Cancer Res, 2011.9(5): p. 564-76.

122. Felsher, D.W., MYC Inactivation Elicits Oncogene Addiction through Both Tumor Cell-Intrinsic and Host-Dependent Mechanisms. Genes Cancer, 2010. 1(6): p. 597-604.

123. Martinelli, G., et al., IKZF1 (Ikaros) deletions in BCR-ABL1-positive acute lymphoblastic leukemia are associated with short disease-free survival and high rate of cumulative incidence of relapse: a GIMEMA AL WP report. J Clin Oncol, 2009. 27(31): p. 5202-7.

124. Iacobucci, I., et al., Identification and molecular characterization of recurrent genomic deletions on $7 p 12$ in the IKZF1 gene in a large cohort of BCR-ABL1positive acute lymphoblastic leukemia patients: on behalf of Gruppo Italiano Malattie Ematologiche dell'Adulto Acute Leukemia Working Party (GIMEMA AL WP). Blood, 2009. 114(10): p. 2159-67.

125. Mullighan, C.G., et al., Genomic analysis of the clonal origins of relapsed acute lymphoblastic leukemia. Science, 2008. 322(5906): p. 1377-80.

126. Bolotin, E., et al., Serum levels of IL-7 in bone marrow transplant recipients: relationship to clinical characteristics and lymphocyte count. Bone Marrow Transplant, 1999. 23(8): p. 783-8.

127. Dean, R.M., et al., Association of serum interleukin-7 levels with the development of acute graft-versus-host disease. J Clin Oncol, 2008. 26(35): p. 5735-41.

128. Kim, D.H., et al., Natural killer or natural killer/T cell lineage large granular lymphocytosis associated with dasatinib therapy for Philadelphia chromosome positive leukemia. Haematologica, 2009. 94(1): p. 135-9.

129. Heltemes-Harris, L.M., et al., Ebfl or Pax5 haploinsufficiency synergizes with STAT5 activation to initiate acute lymphoblastic leukemia. The Journal of Experimental Medicine, 2011. 208(6): p. 1135-1149.

130. Palella, F.J., Jr., et al., Declining morbidity and mortality among patients with advanced human immunodeficiency virus infection. HIV Outpatient Study Investigators. N Engl J Med, 1998. 338(13): p. 853-60.

131. Klonis, N., et al., Artemisinin activity against Plasmodium falciparum requires hemoglobin uptake and digestion. Proc Natl Acad Sci U S A, 2011. 108(28): p. 11405-10. 
132. Cui, L. and X.Z. Su, Discovery, mechanisms of action and combination therapy of artemisinin. Expert Rev Anti Infect Ther, 2009. 7(8): p. 999-1013.

133. Lu, J.J., et al., Dihydroartemisinin accelerates c-MYC oncoprotein degradation and induces apoptosis in c-MYC-overexpressing tumor cells. Biochem Pharmacol, 2010. 80(1): p. 22-30.

134. Shoemaker, R.H., The NCI60 human tumour cell line anticancer drug screen. Nat Rev Cancer, 2006. 6(10): p. 813-23.

135. Holbeck, S.L., J.M. Collins, and J.H. Doroshow, Analysis of Food and Drug Administration-approved anticancer agents in the NCI60 panel of human tumor cell lines. Mol Cancer Ther, 2010. 9(5): p. 1451-60.

136. Szakacs, G., et al., Predicting drug sensitivity and resistance: profiling $A B C$ transporter genes in cancer cells. Cancer Cell, 2004. 6(2): p. 129-37.

137. Naasani, I., et al., FJ5002: a potent telomerase inhibitor identified by exploiting the disease-oriented screening program with COMPARE analysis. Cancer Res, 1999. 59(16): p. 4004-11.

138. Guo, Q., et al., Toxicological studies of gambogic acid and its potential targets in experimental animals. Basic Clin Pharmacol Toxicol, 2006. 99(2): p. 178-84.

139. Zhai, D., et al., Gambogic acid is an antagonist of antiapoptotic Bcl-2 family proteins. Mol Cancer Ther, 2008. 7(6): p. 1639-46.

140. Yi, T., et al., Gambogic acid inhibits angiogenesis and prostate tumor growth by suppressing vascular endothelial growth factor receptor 2 signaling. Cancer Res, 2008. 68(6): p. 1843-50.

141. Dooley, M.A., et al., Mycophenolate versus azathioprine as maintenance therapy for lupus nephritis. N Engl J Med, 2011. 365(20): p. 1886-95. 


\section{APPENDIX. SUPPLEMENTAL DATA}

Supplemental data is made available online in the form of a Microsoft Excel file which contains eight (i through viii) separate worksheets - i) table of contents (comprised of description, legends and experimental notes for the subsequent worksheets ii through viii which contain real experimental data), ii) host factors, iii) gene expression 1, iv) gene expression 2, v) structure, vi) drug screen summary 1, vii) drug screen summary 2, and viii) ATC classification. The Excel file is approximately 3.8Mbytes and can be downloaded from http://etd.uthsc.edu/World-Access/Singh/Supplementaldata.xlsx. 


\section{VITA}

Harpreet Singh was born in Chandigarh, India in 1980. He studied at Shivalik Public School and DAV College, Chandigarh, India, before starting medical school studies in 1997 at Government Medical College, Patiala, India, from where he graduated in 2001. He practiced medicine and taught medical school students at Government Medical College, Chandigarh until 2005. He enrolled at the University of Tennessee Heath Science Center in 2006 as a full-time Ph.D. student in the Integrated Program in Biomedical Sciences. He will obtain his Ph.D. degree in August 2012. 